\title{
The Robotic Molecular Biologist - Automated Processing of Astrobiological Samples for Planetary Missions
}

\author{
by \\ Shubhank Sondhiya \\ A thesis submitted to \\ the Faculty of Graduate and Postdoctoral Affairs \\ in partial fulfillment of \\ the requirement for the degree of
}

Master of Applied Science

in

Mechanical Engineering

Ottawa-Carleton Institute of Mechanical and Aerospace Engineering

Department of Mechanical and Aerospace Engineering

Carleton University

Ottawa, Ontario, Canada

May 2020

Copyright (C)

2020 - Shubhank Sondhiya 
The undersigned recommend to

the Faculty of Graduate and Postdoctoral Affairs

acceptance of the thesis

\title{
The Robotic Molecular Biologist - Automated Processing of Astrobiological Samples for Planetary Missions
}

\author{
Submitted by Shubhank Sondhiya \\ in partial fulfillment of the requirements for the degree of \\ Master of Applied Science
}

Dr. Alex Ellery, Thesis Supervisor

Dr. Ronald E. Miller, Department Chair

Carleton University

2020 


\section{Abstract}

Nanopore based DNA sequencing is becoming more prevalent in life-detection platforms, and the search for life in our solar system is a major focus of planetary exploration. One of the key challenges is the development of a suite of life detection instruments with lower mass and energy requirements.

The aim of this research is the development of a low-mass and robust life detection platform that has the potential to be integrated into future astrobiology space missions and to perform numerical analysis to optimize the platform. To achieve this, a subset of instruments that could be deployed on a Mars rover are examined and a novel stand-alone life detection platform is designed that is equipped with peristaltic pumps, solenoid valves, microfluidic chip, Microbial Activity MicroAssay ( $\mu$-MAMA) and an Automated Nucleic Acid Extraction System (A-NECS). Further, a control algorithm is developed to perform an end-to-end analysis of DNA/RNA extraction and microbial activity detection on the environmental sample collected. A mathematical model for predicting the flow patterns in microfluidics is presented and a CFD analysis of the lysing chamber is conducted to understand turbulent properties, volume density distributions of the dissipated energy and the agitation rate effects. MRF method is used for calculations and two different zones of the lysing chamber are investigated. It is found to improve the accuracy in the prediction of the results without increasing the overall cost, mass, energy consumption of the instrument suite. Further, the results obtained in the thesis indicate that the automated life-detection platform performed significantly well and microbial activity was detected in the sample tested. 


\section{Acknowledgement}

First and foremost, I would like to thank my thesis supervisor, Dr. Alex Ellery, Professor, Mechanical and Aerospace Engineering (MAAE) department, for his valuable instruction, patience, advice and encouragement. He trusted me and gave me the freedom to explore and research the topics that I found interesting and most importantly, corrected me whenever I was wrong. I also wish to gratefully acknowledge Canadian Space Agency (CSA), Dr. Lyle White of McGill University and his research team for their collaboration in this work.

Further, I would thank my close friend, Mayank Vadsola. He and I started the journey of masters together at the same time and learnt a lot from each other over the course of graduate years. It has been an amazing experience working with him.

I am grateful to my fellow roommates not only for their assistance during the difficult times, but also their friendship; these include Revanth Sridhar, Vinay Venkataramanachary and Manoj Goli. I would also like to express my appreciation to Alex Proctor and Kevin Sangster for their assistance in the Machine Shop.

Most importantly, I would like to thank my beloved parents and sister, especially my mother for her endless love, support and encouragement. Finally, the financial support of the FAST grant from Canadian Space Agency for this study is greatly acknowledged. 


\section{Table of Contents}

Abstract iii

Acknowledgement iv

Table of Contents vii

List of Figures xii

List of Tables $\quad$ xiii

List of Abbreviations \& Symbols xiv

1 Introduction 1

2 Background and Literature Review $\quad 7$

2.1 Nanopore-Based Sequencing Technology . . . . . . . . . . . . . . 7

2.2 Types of Nanopores . . . . . . . . . . . . . . . . . . . . . . . . 9

2.2.1 Biological Nanopores . . . . . . . . . . . . . . . . . . 9

2.2.2 Solid-state Nanopores . . . . . . . . . . . . . . . . 9

2.2.3 Hybrid Nanopores . . . . . . . . . . . . . . . . . . . . . . . 10

2.3 Challenges with Nanopore Sequencing . . . . . . . . . . . . . . 11

2.4 Solid-phase Nucleic Acid Extraction . . . . . . . . . . . . . . . . . . 13

2.4.1 Magnetic Bead based Nucleic Acid Purification . . . . . . . 14

2.5 Automated Extraction System . . . . . . . . . . . . . . . . 15

2.5.1 Microfluidic Approach . . . . . . . . . . . . . . . . 17

2.6 Summary . . . . . . . . . . . . . . . . . . . . . 19

3 Automation Methodology 21

3.1 Mathematical Model of Microfluidic System . . . . . . . . . . . . . 21

3.1.1 Continuous Phase . . . . . . . . . . . . . . . . . . . . 22

3.1.2 Dispersed Phase . . . . . . . . . . . . . . . . . . . . . . 24

3.2 Methodology for Automation of the Device . . . . . . . . . . . . . 26

3.2.1 Instrumentation Requirements . . . . . . . . . . . . . . 27 
3.3 Fluidic Manipulation . . . . . . . . . . . . . . . . . 31

3.4 Automation of the Microfluidic Network . . . . . . . . . . . . . . . 32

3.4.1 Non-contact Solenoid Valves . . . . . . . . . . . . . . . . . 32

3.4.2 Splitter Manifold . . . . . . . . . . . . . . . . . . 33

3.4.3 Silicone Rubber Gaskets . . . . . . . . . . . . . . . . . 33

3.4.4 Temperature Controlled Chamber . . . . . . . . . . . . 34

3.5 Microbial Activity MicroAssay ( $\mu$-MAMA) . . . . . . . . . . . 35

3.5.1 First Design . . . . . . . . . . . . . . . . 37

3.5.2 Second Design . . . . . . . . . . . . . . . . . . 39

3.5.3 Final Design . . . . . . . . . . . . . . . . 41

3.6 Nucleic Acid Extraction and Preparation . . . . . . . . . . . . . . 44

3.6.1 Device Fabrication . . . . . . . . . . . . . . . . 46

3.6.2 First Microfluidic Chip with Baffle . . . . . . . . . . . . 46

3.6.3 Final Microfluidic Chip without Baffle . . . . . . . . . . . . 49

3.7 Summary . . . . . . . . . . . . . . . . . . . 50

4 CFD Modeling and Simulations $\quad 52$

4.1 Problem Description . . . . . . . . . . . . . . . 53

4.2 Two Compartment Representation . . . . . . . . . . . . . . . 53

4.2.1 Determination of Two Compartment Parameters . . . . . . 54

4.3 Geometry of the Model . . . . . . . . . . . . . . . . . 55

4.4 Grid Generation . . . . . . . . . . . . . . . . . 55

4.5 Governing Equations . . . . . . . . . . . . . . . 57

4.6 MRF Method for CFD Simulations . . . . . . . . . . . . . . 58

4.7 Model Validation . . . . . . . . . . . . . . . . . . . 59

4.8 Summary . . . . . . . . . . . . . . . . . . . 67

5 Results and Discussion $\quad 68$

5.1 Experimental Results . . . . . . . . . . . . . . . . . 68

$5.1 .1 \mu$-MAMA Device Analysis . . . . . . . . . . . . . . 69

5.1.1.1 Experimental Setup . . . . . . . . . . . 69

5.1.1.2 Results: Glacial Ice and Cryoconite Hole . . . . . . 70

5.1.1.3 Results: Gypsum Hill Springs . . . . . . . . . . . 73

5.1.1.4 Results: Lost Hammer Springs . . . . . . . . . . 73

$5.1 .2 \quad$ A-NECS Device Analysis . . . . . . . . . . . . . . . 74

5.1.2.1 Experimental Set up . . . . . . . . . . 74

5.1.2.2 Results: Microfluidic Chip with Baffle . . . . . . 76

5.1.2.3 Results: Microfluidic Chip without Baffle . . . . 76

5.2 Computational Fluid Dynamics Results . . . . . . . . . . . . . 77 
6 Conclusion and Future Work $\quad 84$

6.1 Conclusion . . . . . . . . . . . . . . . . . . . . . 84

6.2 Future Work . . . . . . . . . . . . . . . 86

6.3 Applications ..................... 87

$\begin{array}{lr}\text { References } & 88\end{array}$

$\begin{array}{ll}\text { A Drawing Sheets for the 3D Models } & 104\end{array}$

$\begin{array}{ll}\text { B Data and Instruction Sheets } & 108\end{array}$

B.1 Data sheet for W1209 Temperature Control Module . . . . . . . . . 108

B.2 Instructions Sheet for Dragon Skin ${ }^{\mathrm{TM}}$ Series . . . . . . . . . . . . . 111

$\begin{array}{lr}\text { C IAC Conference Paper } & 113\end{array}$ 


\section{List of Figures}

1.1 Nanopore-based sequencing . . . . . . . . . . . . . . . . 2

1.2 An Overall flow architecture of the instrumentation suite. . . . . . . 3

1.3 Mechatronics architecture of the instrumentation suite depicting input/output to the system. . . . . . . . . . . . . 4

2.1 Side and top views of heptameric $\alpha$-hemolysin toxin, a biological nanopore, from Staphylococcus aures . . . . . . . . . . . . 10

$2.2 \alpha$ HL heteroheptamer with a $3 \mathrm{kbp}$ dsDNA attached via a 12 -nucleotide oligomer to one protein subunit . . . . . . . . . . . . . 11

2.3 MinION Mk 1B with R9 flowcell . . . . . . . . . . . . . . . . 16

2.4 Claremont SimplePrep X1 automated lysis and nucleic acid extraction platform .......................... 17

3.1 Laminar flow in a straight circular tube with radius . . . . . . . . . 22

3.2 A circuit diagram schematic of the astrobiology instrumentation suite. 28

3.3 Chemical formula for alamarBlue dye. . . . . . . . . . . . . . . 30

3.4 Image of an RP-TX series micropump used in the micro-life system; dimensions $33 \mathrm{~mm} \times 12 \mathrm{~mm} \times 21.5 \mathrm{~mm} \ldots \ldots . \ldots 31$

3.5 8-Port Splitter manifold with Microassay; scale: $10 \mathrm{~mm}$ (height) $\times$ $35 \mathrm{~mm}$ (diameter). . . . . . . . . . . . . . . 33

3.6 Dragon Skin ${ }^{\mathrm{TM}} 30$ high performance platinum cure liquid silicone

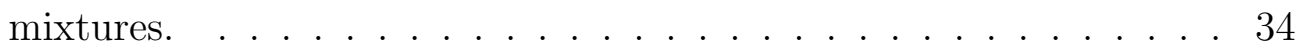


3.7 Temperature control chamber with temperature control module, a ceramic PTC heater and thermostat sensor; scale: $200 \mathrm{~mm} \times 135$ $\mathrm{mm} \times 165 \mathrm{~mm} \ldots \ldots \ldots \ldots \ldots \ldots \ldots$

3.8 Base plate for the first $\mu$-MAMA model with tight seal barbed fittings; scale: $46 \mathrm{~mm} \times 46 \mathrm{~mm} \times 5 \mathrm{~mm} \ldots \ldots \ldots$. . . . . . 37

3.9 Top plate for the first $\mu$-MAMA model; scale: $56 \mathrm{~mm} \times 46 \mathrm{~mm} \times$ $10 \mathrm{~mm} \ldots \ldots \ldots \ldots$

3.10 Silicone gasket for the first $\mu$-MAMA model; scale: $39 \mathrm{~mm} \times 39$ $\mathrm{mm} \times 0.5 \mathrm{~mm} \ldots \ldots \ldots \ldots \ldots$

3.11 Base plate of the second $\mu$-MAMA model with tight seal barbed fittings; scale: $13 \mathrm{~mm}$ (height) $\times 68 \mathrm{~mm}$ (diameter). . . . . . . . . 40

3.12 Assembly of the first $\mu$-MAMA model; scale: $1 \mathrm{~mm}$ (height) $\times 60$ mm (diameter). . . . . . . . . . . . . . . . . 40

3.13 Channel blockage in the second design of $\mu$-MAMA model; scale: $13 \mathrm{~mm}$ (height) $\times 68 \mathrm{~mm}($ diameter $) \ldots \ldots . . . . . . . . .441$

$3.143 \mathrm{D}$ design model of the final $\mu$-MAMA base plate; scale: $17 \mathrm{~mm}$ (height) $\times 68 \mathrm{~mm}$ (diameter) . . . . . . . . . . . . . . 42

$3.153 \mathrm{D}$ design model of the final $\mu$-MAMA top plate; scale: $5 \mathrm{~mm}$ (height) $\times 68 \mathrm{~mm}($ diameter $) \ldots \ldots \ldots . \ldots . \ldots . \ldots 42$

3.16 Assembly of the final $\mu$-MAMA design model; scale: scale: $24 \mathrm{~mm}$ (height) $\times 68 \mathrm{~mm}$ (diameter) .

3.17 Silicone gasket for the final $\mu$-MAMA model; scale: $1 \mathrm{~mm}$ (height) $\times 68 \mathrm{~mm}$ (diameter).

3.18 Zoomed-in view of the final $\mu$-MAMA sub-unit; scale: $200 \mathrm{~mm} \times$ $135 \mathrm{~mm} \times 165 \mathrm{~mm} \ldots \ldots \ldots \ldots \ldots$

3.19 Top and side views of the first microfluidic chip with baffle dimensions; all dimensions are in mm. . . . . . . . . . . . . . . . . 47

$3.203 \mathrm{D}$ printed bottom part of the $1^{\text {st }}$ microfluidic chip with baffle; all dimensions are in $\mathrm{mm}$. 
$3.213 \mathrm{D}$ printed top part of the $1^{\text {st }}$ microfluidic chip with baffle; scale: $80 \mathrm{~mm} \times 45 \mathrm{~mm} \times 3 \mathrm{~mm}$.

3.22 Assembly of the $1^{\text {st }}$ microfluidic chip with baffle; scale: $80 \mathrm{~mm} \times 45$ $\mathrm{mm} \times 10 \mathrm{~mm}$.

3.23 Top and side views of the final microfluidic chip with baffle dimensions; all dimension are in mm. . . . . . . . . . . . . . . . . 49

3.24 Assembly of the second microfluidic chip without baffle; scale: 100 $\mathrm{mm} \times 50 \mathrm{~mm} \times 15 \mathrm{~mm} \ldots \ldots \ldots \ldots$

4.1 Model geometry for CFD simulations. . . . . . . . . . . . . . . 55

4.2 Computational domain discretization into a unstructured tetrahedral mesh. . . . . . . . . . . . . . . . . . 56

4.3 Y-velocity distribution along the magnet zone $\mathrm{x}$ axis. . . . . . . . . 57

4.4 Velocity vectors plot in the XZ plane from Alexopoulos et al. work 60

4.5 Velocity vectors plot in the XY plane from Alexopoulos et al. work 60

4.6 Energy dissipation rate contour in the XZ plane from Alexopoulos et al. work . . . . . . . . . . . . . . . . . 6 61

4.7 Energy dissipation rate contour in the XY plane from Alexopoulos et al. work . . . . . . . . . . . . . . . . 6 61

4.8 Velocity magnitude contour plot obtained from $k-\varepsilon$ realisable

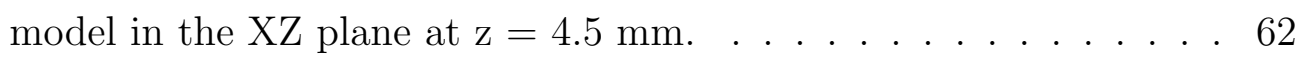

4.9 Total pressure contour plot obtained from $k-\varepsilon$ realisable model in the XZ plane at $\mathrm{z}=4.5 \mathrm{~mm} . \ldots \ldots \ldots$. . . . . . . . 62

4.10 Turbulent kinetic energy contour plot obtained from $k-\varepsilon$ realisable model in the XZ plane at $\mathrm{z}=4.5 \mathrm{~mm} . \ldots . . . . . . . .663$

4.11 Velocity vector plot obtained from $k-\varepsilon$ realisable model in the XZ plane at $\mathrm{z}=4.5 \mathrm{~mm} \ldots \ldots \ldots \ldots$

4.12 Velocity magnitude contour obtained from $k-\omega$ SST model in the $\mathrm{XZ}$ plane at $\mathrm{z}=4.5 \mathrm{~mm} \ldots \ldots \ldots \ldots$. . . . . . . . . 64 
4.13 Total pressure contour obtained from $k-\omega$ SST model in the XZ plane at $\mathrm{z}=4.5 \mathrm{~mm} \ldots \ldots \ldots \ldots \ldots$

4.14 Turbulent kinetic energy contour obtained from $k-\omega$ SST model in the XZ plane at $\mathrm{z}=4.5 \mathrm{~mm}$.

4.15 Streamline plot obtained from $k-\omega$ SST model in the XZ plane at $\mathrm{z}=4.5 \mathrm{~mm} \ldots \ldots \ldots \ldots \ldots$

4.16 Phase 2 volume of fluid contour obtained from $k-\omega$ SST model. . 66

4.17 Phase 2 volume of fluid contour obtained from $k-\varepsilon$ realisable model. 67

$5.10 .20 \mu \mathrm{m}$ membrane filter used for filtration stage; scale: $30 \mathrm{~mm}$ (height) $\times 30 \mathrm{~mm}($ diameter $) \ldots \ldots . \ldots 70$

5.2 Assembly of the instrumentation suite; scale: $35 \mathrm{~cm} \times 31 \mathrm{~cm} \times 17$

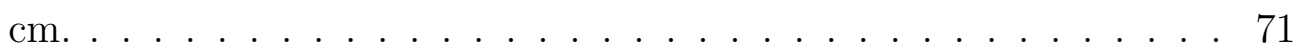

5.3 A flowchart of the simulation algorithm. . . . . . . . . . . . 72

5.4 3D model of the magnet housing; scale: $9.85 \mathrm{~mm}$ (height) $\times 21.10$ mm (diameter). . . . . . . . . . . . . . . . 75

5.5 3D model of the DC motor housing; scale: $78 \mathrm{~mm}$ (height) $\times 23.50$ mm (diameter). . . . . . . . . . . . . . . . 75

5.6 Assembly of the disc magnet coupled with DC motor shaft; scale: $83 \mathrm{~mm}$ (height) $\times 23.50 \mathrm{~mm}$ (diameter) . . . . . . . . 77

5.7 Cumulative energy dissipation for run 1 at the magnet zone. . . . . 79

5.8 Cumulative energy dissipation for run 2 at the magnet zone. . . . . 79

5.9 Cumulative energy dissipation for run 3 at the magnet zone. . . . . 80

5.10 Effect of agitation rate on the turbulent kinetic energy for $k-\varepsilon$ realisable model. . . . . . . . . . . . . . . . . . . 81

5.11 Effect of agitation rate on the turbulent viscosity for $k-\varepsilon$ realisable model. ............................ 81

5.12 Effect of agitation rate on the energy dissipation rates for $k-\varepsilon$ realisable model. 
5.13 Plot of volume density distribution of the energy dissipation rates for different agitation rates of $k-\varepsilon$ realisable model. . . . . . . . . 82 


\section{List of Tables}

3.1 Micropump parameters and their specifications. . . . . . . . . . . 29

4.1 Grid independent study for different element sizes. . . . . . . . . . . 56

5.1 Summary of the tests performed for the $\mu$-MAMA unit. . . . . . . . 69

5.2 Brushed DC motor attributes. . . . . . . . . . . . . 76

5.3 Summary of the different run specifications. . . . . . . . . . . 78 


\title{
List of Abbreviations \& Symbols
}

\author{
List of Abbreviations \\ $\alpha$-HL Alpha Hemolysin \\ $\mu$-MAMA Microbial Activity MicroAssay \\ $\mu$-TAS Miniaturized Total Analysis System \\ A-NECS Automated Nucleic Acid Extraction System \\ bya Billion Years Ago \\ CFD Computational Fluid Dynamics \\ CMOS Complementary Metal-Oxide Semiconductor \\ DC Direct Current \\ DNA Deoxyribo Nucleic Acid \\ DNase Deoxyribonuclease \\ dsDNA Double Stranded Deoxyribo Nucleic Acid \\ GC-MS Gas Chromatography-Mass Spectrometry \\ ICCD Intensified Charge Coupled Device \\ IR Infrared Radiation \\ kbp Kilo-base Pair
}


LED Light Emitting Diode

MP1 MicroPump1

MP2 MicroPump2

mRNA Messenger Ribo Nucleic Acid

NTC Negative Temperature Coefficient

ONT Oxford Nanopore Technologies

PC Polycarbonate

PCR Polymerase Chain Reaction

PDE Partial Differential Equations

PDMS Polydimethylsiloxane

PEEK Polyether Ether Ketone

PFPE Perfluoropolyether

PoC Proof-Of-Concept

ppb Parts Per Billion

ppm Parts Per Million

PSD Particle Size Distribution

PTC Positive Temperature Coefficient

PTFE Polytetrafluoroethylene

qPCR Quantitative Polymerase Chain Reaction

RANS Reynolds-averaged Navier-Stokes

RNA Ribo Nucleic Acid 
RNase Ribonuclease

RPM Revolutions Per Minute

rRNA Ribosomal Ribo Nucleic Acid

RSL Recurring Slope Linea

SAM Sample Analysis at Mars

SETG Search for Extraterrestrial Genome

SiN Silicon monoNitride

ssDNA Single Stranded Deoxyribo Nucleic Acid)

SST Shear Stress Transport

SV1 SolenoidValve1

SV2 SolenoidValve2

TE-Buffer Tris EDTA Buffer

tRNA Transfer Ribonucleic Acid

USP U.S Pharmacopoeia

VOF Volume Of Fluid

\section{List of Symbols}

$\bar{u} \quad$ Average velocity, $\mathrm{m} / \mathrm{s}$

$\delta \quad$ Droplet length per inter-distance, $m$

$\triangle P \quad$ Pressure gradient, $P a$

$\frac{\partial P}{\partial x} \quad$ Pressure Gradient along x-direction, $\mathrm{Pa} / \mathrm{m}$

$\mu_{c} \quad$ Dynamic viscosity of continuous phase, $P a-m-s$ 


\begin{tabular}{|c|c|}
\hline$\mu_{d}$ & Dynamic viscosity of dispersed phase, $P a-m-s$ \\
\hline$\mu_{d}$ & Dynamic viscosity of dye, $\mathrm{kg} / \mathrm{m}-\mathrm{s}$ \\
\hline$\mu_{w}$ & Dynamic viscosity of water, $\mathrm{kg} / \mathrm{m}-\mathrm{s}$ \\
\hline$\rho_{d}$ & Density of dye, $\mathrm{kg} / \mathrm{m}^{3}$ \\
\hline$\rho_{w}$ & Density of water, $\mathrm{kg} / \mathrm{m}^{3}$ \\
\hline$\sigma$ & Interfacial tension coefficient, $N / m$ \\
\hline$\sigma_{k}$ & Experimental constant in equation $\varepsilon$ \\
\hline$\sigma_{k}$ & Experimental constant in equation $k$ \\
\hline$\tau_{i j}^{(t)}$ & Turbulent momentum flux (Reynold Stress) \\
\hline$\varepsilon$ & Energy dissipation rate, $m^{2} / s^{3}$ \\
\hline$\varepsilon_{\min }$ & Minimum energy dissipation rate, $\mathrm{m}^{2} / \mathrm{s}^{3}$ \\
\hline$A$ & Area of cross-section, $m^{2}$ \\
\hline$C_{a}$ & Capillary number \\
\hline$D$ & Diameter of the tube, $m$ \\
\hline$d V$ & Potential drop, volts \\
\hline$H(x)$ & Heaviside function \\
\hline$L$ & Length of tube between two points, $m$ \\
\hline$l_{d}$ & Droplet length, $m$ \\
\hline$n_{i}$ & Volume density distribution of the energy dissipation rate \\
\hline$N_{t o t}$ & Total number of grid cells \\
\hline$b_{1}$ & Pressure at point $1, P a$ \\
\hline
\end{tabular}




$\begin{array}{ll}p_{2} & \text { Pressure at point } 2, P a \\ Q_{c} & \text { Volumetric flow rate for continuous phase, } \mathrm{m}^{3} / \mathrm{s} \\ Q_{d} & \text { Volumetric flow rate for dispersed phase, } \mathrm{m}^{3} / \mathrm{s} \\ r_{0} & \text { Radius of the circular cross-section, } \mathrm{m} \\ R_{h} & \text { Microfluidic flow resistance, } \mathrm{Pa}-\mathrm{s} / \mathrm{m}^{3} \\ u^{\prime} & \text { Cluctuation velocity, } \mathrm{m} / \mathrm{s} \\ u_{c} & \text { Upper bound, } \mathrm{m} / \mathrm{s} \\ u_{c}^{*} & \text { Maximum centre-line velocity, } \mathrm{m} / \mathrm{s} \\ u_{m a x} & \text { Average velocity, } \mathrm{m} / \mathrm{s} \\ V & \end{array}$




\section{Chapter 1}

\section{Introduction}

Discoveries made by Mars Global Surveyor [1] and Mars Exploration Rovers [2] on evidence of water activity on Mars prompted intense interest in the planet's in situ exploration of biomarker chemical signatures indicative of extinct life in subsurface water or ice deposit. In addition, the OMEGA instrument's surface spectral data provided detailed mineralogical maps, highlighting regions of past water activity where life may have thrived in early periods in Martian history [3]. Hayes et al. [4] stated that Europa and Enceladus, in the medium to long term are the primary targets for astrobiology investigations other than Mars.

Extremely cold temperatures characterize these primary targets for astrobiology exploration, and, as such, the Earth's polar regions may be the best terrestrial analogues. These targets, based on the current knowledge of extremophile microbiology, are potentially capable of hosting microbial life and ecosystems either at present or in the past. For example, there was considerable evidence on Mars indicating that the planet was warmer and wetter [5] prior to $\sim 3.5$ bya. Nevertheless, the recent report of surface brine water at several recurring slope linaea (RSL) sites on Mars [6] now opens up the possibility that existing microbial life (which would most likely be cold-adapted and halophilic) may be present at these sites and will almost certainly be the targets of future missions in the mid-2020s and beyond, including potential sample return missions. Similarly, exciting discoveries over the 
last 5-10 years indicate the existence of cold, salty oceans under the ice covers of Europa and Enceladus [7-11] that could also support extantmicrobial ecosystems based on our knowledge of similar salty cryoenvironments on Earth [12]. Thus, DNA-related testing becomes evident as it is one of the essential components of life which stores and transmits genetic information.

DNA is recognised as the blueprint or the foundation containing the information needed to encrypt proteins and other components of cells. Nucleic acids are seen as unambiguous signs of extant life and are an excellent candidate for detection of biosignatures. Nanopore sequencing technologies can detect these

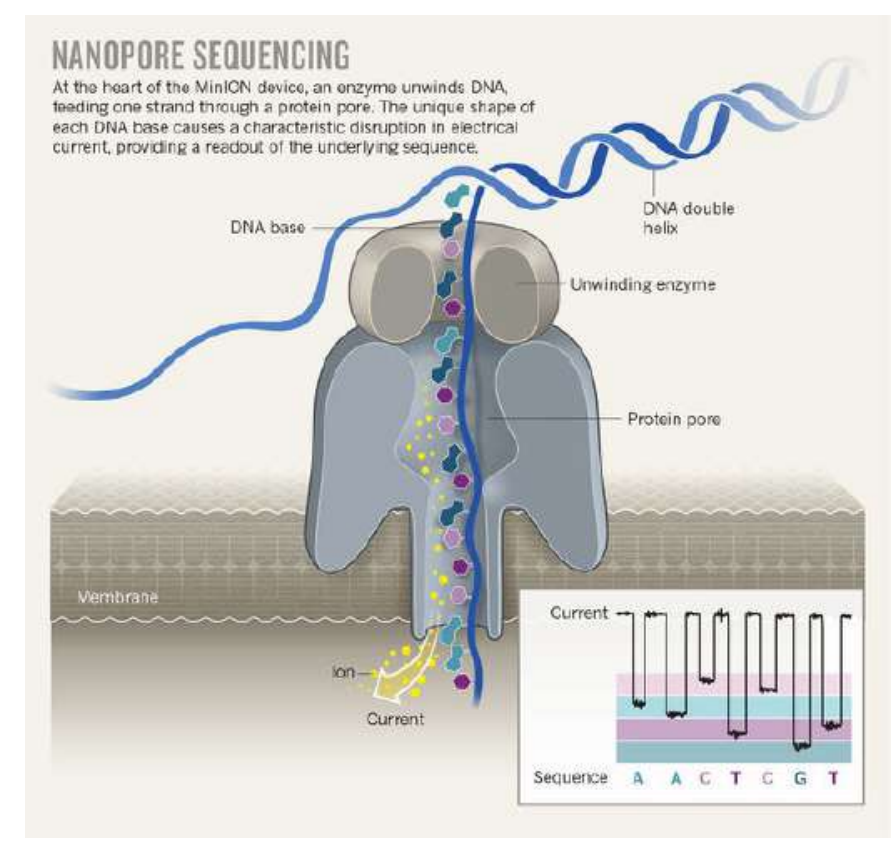

Figure 1.1: Nanopore-based sequencing [13].

nucleic acids. Nanopore-based DNA sequencing technology has become one of the most interesting and engaging third-generation technologies due to its outstanding label-free, amplification-free, great reading length, high throughput that makes it compelling for high-quality gene sequencing applications, such as de novo sequencing, high-resolution analysis of chromosomal structure variation, and longrange haplotype mapping [14]. There are, however, certain undeniable drawbacks [15][16], such as the nanopore's rigorous environmental requirements (i.e. temperature electrolyte concentration and $\mathrm{pH}$ ) to maintain its biological activities. 
The components of an ideal commercial sequencing system using electrical measurements would consist of a disposable detector chip that contains a range of nanopores with the required integrated microfluidic and electronic probes, a portable system that controls the chip's fluids and the electronic elements, and processes the raw sequence data. Assuming that one chip is required to sequence a sample from analogue sites with the complexity of the genome, the cost of the complete genomic sequencing will include the cost of preparing the genomic DNA from a biological sample, the cost of the nanopore device and the cost of the disposable detector chip. An overall flow architecture and the mechatronics architecture that shows the input/outputs of the system are shown in figures (1.2) and (1.3).

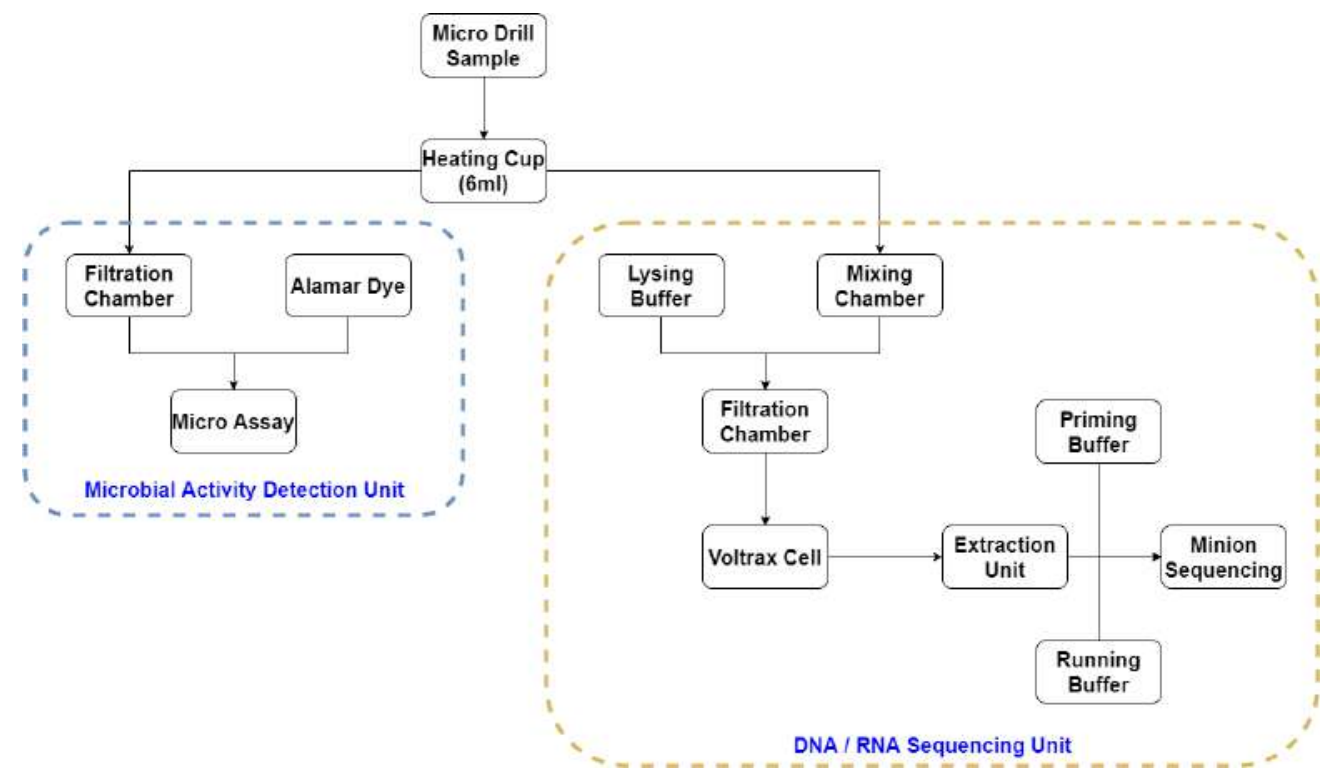

Figure 1.2: An Overall flow architecture of the instrumentation suite.

Although life detection is a primary driver of planetary exploration, since the Viking Missions to Mars in the 1970s [17-19], no direct life detection instrumentation has been included on an astrobiology mission payload. The scientific instruments currently available to space missions in astrobiology are focused on identifying habitable environments or detecting biosignatures. To date, these instruments remain high mass, have high operating costs, are large in size, and have high demands on energy. Such instruments are completely unsuitable for mis- 


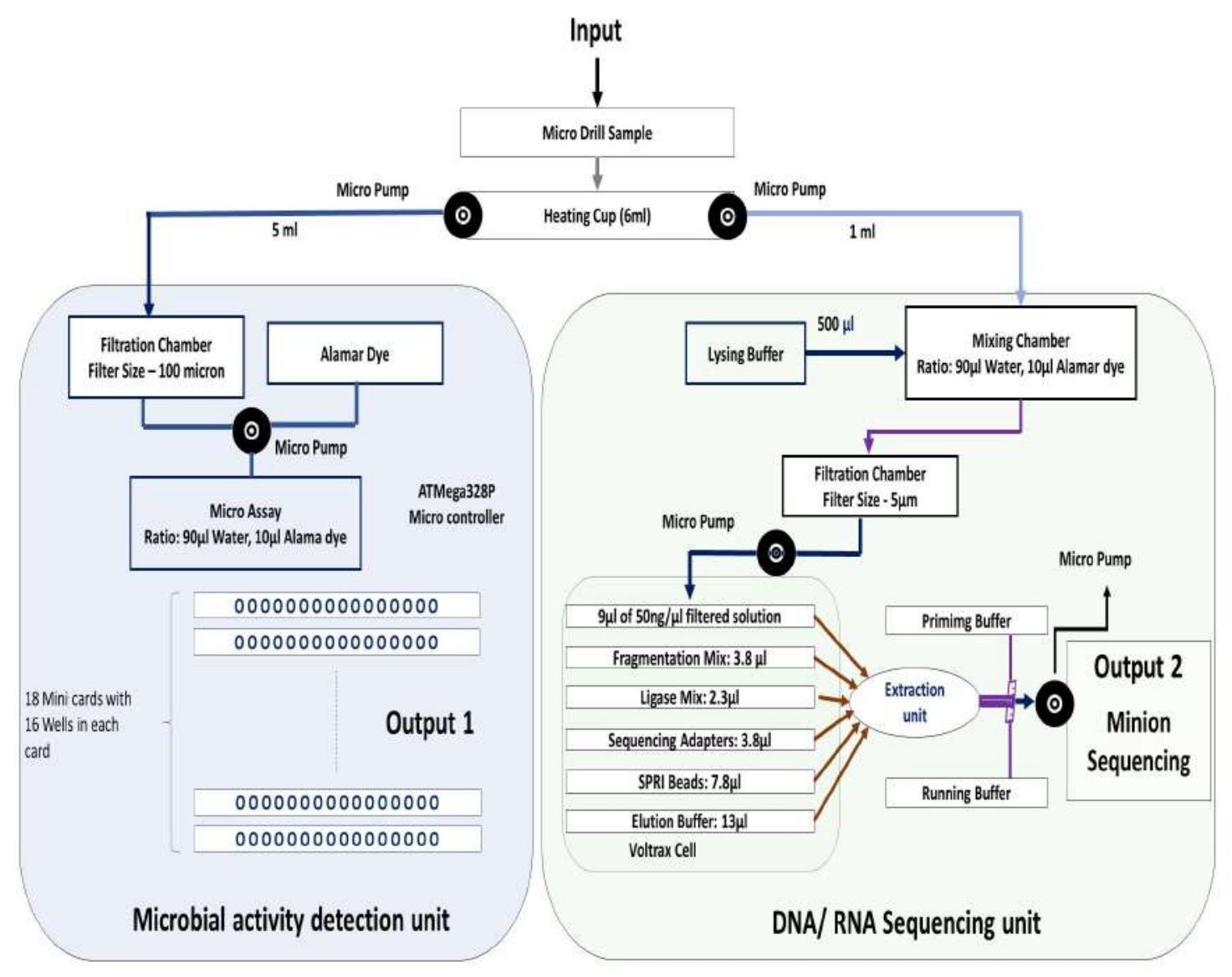

Figure 1.3: Mechatronics architecture of the instrumentation suite depicting input/output to the system.

sions to locations like Europa or Enceladus for which lander packages are likely to be strictly restricted. There are advantages of using multiple small scientific instruments over fewer larger instruments, even for Mars missions. In addition, the difficulties in defining biomarkers attest to the need to deploy more specific astrobiological instruments [20]. Therefore, to learn as much as possible about these environments which may only contain trace quantities of organic materials, the need arises for a fully automated instrumentation suite capable of sensitive analysis of a wide range of organic and inorganic molecules.

The aim of this research is therefore to use and integrate low mass, low cost, and robust next generation miniaturized scientific instrumentation for biosignature detection, and microbiology techniques in a novel context for identifying biosignature and characterizing viable microbial life in a high fidelity analogue environment in the Canadian high Arctic. Such a microbial life detection platform 
which can be independently controlled via computer control and attached to surface rover platforms or penetrators could be conceptually used in future missions to Mars, Europa, Enceladus or any other planetary target. Additionally, such a platform also has clear applications for rapid community metagenomics analysis and microbial activity assessment in environmental field sites, including extreme sites such as the high Arctic and Antarctic [21][22].

The first outcome of this research is the development of a mathematical model of the major microfluidic elements capturing the fluid flow across the channels. These models will serve as a platform for selecting the appropriate instruments and integrating multiple analytical and sample processing functions, such as sampling, filtering, dilution, mixing and separation on microfluidic assay and nanopore instruments. The second outcome is the development of a CFD model to study flow behaviour, circulation patterns, vortical structures, Reynold stresses, and turbulent kinetic energy associated with the flow in the mixing chamber. This research led to the development of the fully automated prototype, which is found to improve the accuracy of prediction results without increasing the overall cost of the instrument suite and a portion of this research was presented and published in the proceedings of the IAC 2019 Conference [23].

The main objectives of this research is to provide the following novel contributions:

1. A CFD model to develop an understanding of the interaction between fluid and structure, and to add theoretical depth to the instrument design.

2. A fully automated proof-of-concept prototype for end-to-end analysis of DNA/RNA preparation and sequencing by using the circuitry's novel $\mu$ MAMA design and Oxford Nanopore Instruments.

In this thesis, there are six chapters. After a brief introduction in Chapter 1, Chapter 2 reviews the existing literature on microfluidic systems, including DNA/RNA sequencing technologies, instrumentation and material required for integration and manufacturing, CFD modelling to study flow behaviours and other 
parameters associated with flow. In Chapter 3, the methodology used to develop a mathematical model is introduced, the design process of the different sub-units and the steps involved in automating the system are discussed. Chapter 4 highlights the CFD analysis and the study of flow-related parameters. The experimental results obtained from field tests for $\mu$ - MAMA, and the automated nucleic acid extraction instrumentation suite are discussed in Chapter 5. Finally, Chapter 6 summarizes all the work presented in this thesis together to provide concluding remarks on the application of work to the development of the fully automated instrument suite for biosignature and microbial activity detection. Recommendations for future work, including additional capabilities that can be derived from the research presented, are also discussed in this chapter. 


\section{Chapter 2}

\section{Background and Literature}

\section{Review}

The available literature suggests that the duration for Mars missions is likely to range from 1.5 to 3 years, with 12 to 24 months of that time spent in transit between the planets, based on current propulsion technologies and planetary orbital dynamics. In response to spaceflight, the human immune response becomes dysregulated [24], and microbial pathogenicity may increase during spaceflight [25]. The ongoing microbial evolution may have a profound impact on crew health, as microbiome stability and dynamics on Earth [26][27] are known to have significant effects on human health. Considering the time required to reach Mars, intervention from Earth during the Mars mission will be limited to electronic communication, meaning that any analysis or monitoring to be performed must be done in situ.

\subsection{Nanopore-Based Sequencing Technology}

The nanopore-based DNA sequencing was first proposed by Church et al. in 1995 [28]. One year later, Kasianowicz et al. [29] demonstrated the detection of the single-stranded DNA (ssDNA) electronic behaviour passing through an $\alpha$-hemolysin (2.6nm in diameter) nanopore, which marked the beginning of the nanopore-based DNA sequencing field. Biological nanopores have many benefits 
[15][16], such as the dimensional reproducibility, genetic or chemical modification process compatibility, and relatively slower DNA translocation velocity through nanopores.

In the past several years, both research and commercial enterprises have begun developing DNA sequencers which can be used in the field for on-site sequencing studies [30-33]. Among these is the handheld MinION sequencer from Oxford Nanopore Technologies (ONT) that measures ionic current changes as DNA passes through an array of protein nanopores that are embedded in a special polymer membrane [34]. As DNA passes through the nanopore, it causes a characteristic current change for each nucleotide group, allowing sequencing of the bases. This massively parallel single molecule real-time sequencing process can generate continuous read lengths $>100 \mathrm{~kb}$ with a total run output of $>10 \mathrm{~Gb}$ [35]. Since this instrument allows for direct sequencing of native DNA and avoids bias in PCR, it has beneficial applications in many sequencing situations and extremophile environments [36]. For example, ambient conditions in extremely cold and arid locations can preserve fragmented strands of ancient DNA over very long timescales. Strands of ancient DNA recovered from environmental samples are typically only 100-500 bp in length [37], thus the long read lengths (> $10 \mathrm{~kb}$ ) generated by third-generation sequencing techniques can improve genomic analysis by helping to distinguish free floating DNA recovered from intact cells in the environment. Unlike traditional sequencing methods, there is no need for prior knowledge of molecular structure and comparison. These attributes make MinIOn a prime candidate for planetary missions, like Mars landers, as an astrobiological life or biosignature detection instrument.

Astrobiologists have long used polar field sites as analogues for low-biomass environments as a testbed for life detection strategies, and the incorporation of MinION in life detection strategy trials provides some insight into MinION's performance in remote and challenging environments. Johnson et al. [22] performed a technical performance and endurance trial of the MinION platform in Antarc- 
tica. In addition, the incorporation by Goordial et al. [12] of MinION basedmetagenomics along with culturing as a proof-of-concept life detection system in the Canadian High Arctic demonstrated the potential usefulness of MinION sequencing in a field setting.

\subsection{Types of Nanopores}

Nanopores are classified into three classes (1). Biological nanopores, (2). Synthetic nanopores and (3). Hybrid nanopores.

\subsubsection{Biological Nanopores}

Over the years, relatively larger channels were explored for nanopore detection purposes as compared to ion channels. For all the biological pores the substrate of choice is planar lipid membranes, liposomes or polymer membranes housed in an electrochemical chamber. By employing standard molecular biology techniques, large-scale production and purification of different channel proteins are possible. In most cases the purified pores of the channel are homogeneous from various batches. Additionally, due to the available crystal structure of several channel proteins, explicit engineering of the channel pores via site-directed mutagenesis is possible [38].

\subsubsection{Solid-state Nanopores}

Due to its unique properties, including well-defined geometries and dimensions, mechanical robustness, ease of modification and compatibility with various electronic or optical measuring techniques, solid-state nanopores have emerged as a versatile alternative to biological nano-pores. Depending on the experimental requirements, the diameter of the solid-state nanopores can be precisely controlled ranging from subnanometers to hundreds of nanometers. In general, solid-state nanopores exhibit superior chemical and thermal stability over lipid membranes 

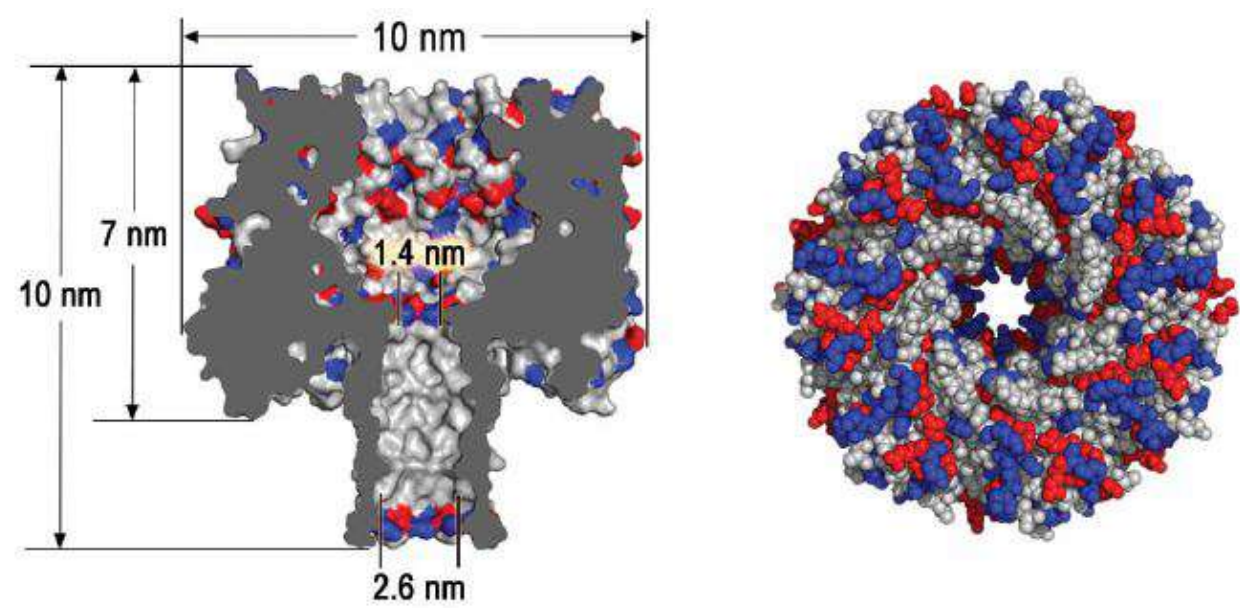

Figure 2.1: Side and top views of heptameric $\alpha$-hemolysin toxin, a biological nanopore, from Staphylococcus aures [39].

in dielectric materials such as SiN. However, their stability depends on the conditions that were used to form these pores [40]. In recent years, solid-state nanopores have opened the door to a wide range of potential applications in DNA sequencing [41], monitoring protein interactions [42], controlling molecular transport, and manufacturing nanofluidic devices [43].

\subsubsection{Hybrid Nanopores}

The concept of hybrid pores was demonstrated by inserting a $\alpha$-hemolysin channel in $\mathrm{SiN}$ pores [44]. $\alpha$-Hemolysin has an atomicly accurate structure and potential for site-specific genetic engineering or chemical modifications. But $\alpha$-hemolysin relies on a fragile lipid bilayer membrane for mechanical support, which significantly restricts its integration into wafer-scale devices. However, this limitation is circumvented by placing a biological pore inside a mechanically robust solid-state nanopore thus creating a hybrid pore with the advantages of both worlds. The hybrid system was shown to be stable for several days under observation [44]. However, the biological pore deforms once in contact with the solid-state pore, loses the ability to discriminate between single nucleotides, and leakage currents are significant as ions can still flow through the regions between the solid-state and biological pore due to an imperfect seal. These factors are limiting this tech- 


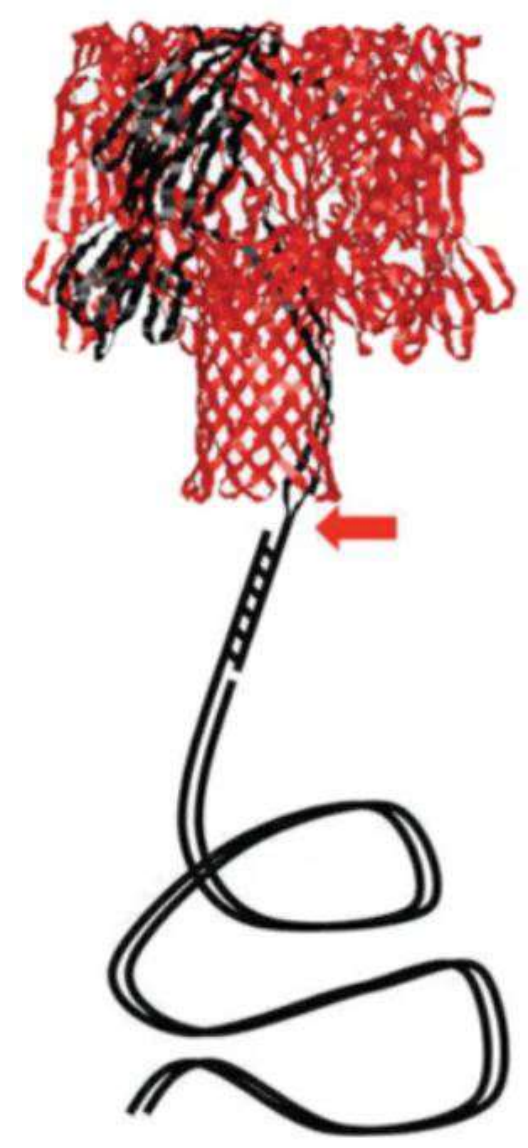

Figure 2.2: $\alpha \mathrm{HL}$ heteroheptamer with a $3 \mathrm{kbp}$ dsDNA attached via a 12nucleotide oligomer to one protein subunit [44].

nology/approach. An example of a hybrid nanopore can be seen in figure (2.2).

\subsection{Challenges with Nanopore Sequencing}

(a) Speed of translocation: A bottleneck in all nanopore sequencing techniques is the ultrafast translocation of DNA, far beyond the limits of current technologies of electronic detection. Speeds of $>10 \mathrm{nt} / \mu \mathrm{s}$ in solid state pores and $>1 \mathrm{nt} / \mu \mathrm{s}$ in $\alpha$-hemolysin and MspA pores were reported [16].

(b) Spatial resolution: Typically the nanochannels investigated are several nanometers long; as a result, at any given point 10-20 nucleotides reside within the lumen of the channel, making it difficult to distinguish the signal resulting from a single nucleotide. 
(c) Temporal resolution: DNA motion is stochastic and kinetic translocation fluctuations occur. Furthermore, non-specific interactions with the channel wall result in a wide distribution of dwelling times and different electrical signatures for identical length and DNA sequences.

(d) Accuracy rates: For the successful application of nanopores, the length of DNA that can be sequenced and the accuracy rate of reading the bases are of paramount importance. This is a major obstacle that has not yet been addressed in any systems to date. The $\alpha$-hemolysin pore reported a $10-25 \%$ error, while the MspA pore relies on a computer reference map to assign individual nucleotides. An error rate of 4 percent was reported using the Oxford Nanopore Technologies.

Before a DNA, RNA, or protein can be sequenced using nanopore-based sequencing technology, it is important to use relevant methods for nucleic acid extraction and concentration to allow high-yield isolation of high-purity nucleic acids [45] - this is the most crucial step in molecular biology. DNA, RNA, and protein can be isolated for analytical or preparatory purposes from any biological material such as living or preserved tissues, cells, virus particles, or other samples [45]. Successful purification of nucleic acid generally requires four important steps: effective cell tissue disruption; denaturation of nucleoprotein complexes; nuclease inactivation, for example, RNase for RNA extraction and DNase for DNA extraction; away from contamination [46]. The target nucleic acid should be free of contaminants including protein, carbohydrate, lipids, or other nucleic acid, for example, DNA free of RNA or RNA free of DNA [47]. On the other hand, RNA is an unstable molecule and once extracted from the cell or tissue has a very short half-life [48]. There are several types of naturally occurring RNA including ribosomal RNA (rRNA) (80\%-90\%), messenger RNA (mRNA) (2.5\%-5\%) and transfer RNA (tRNA) [47]. Special care and precautions are required for RNA isolation as it is susceptible to degradation [47][49]. RNA is especially unstable because of the ubiquitous existence of RNases that are enzymes present in blood, all tissues, 
as well as most bacteria and fungi in the environment [47][48].

Currently, there are a number of specialized method for extracting pure DNA, RNA, or protein. They are generally divided into solution-based or column-based protocols. Most of these protocols have been developed into commercial kits that facilitate the extraction of biomolecules.

\subsection{Solid-phase Nucleic Acid Extraction}

Solid-phase nucleic acid purification can be found in most of the commercial extraction kits available on the market. This enables quick and efficient purification compared to conventional methods [50]. Many of the problems associated with liquid-liquid extraction, such as incomplete phase separation can be avoided. The solid phase system absorbs nucleic acid in the extraction process, depending on the $\mathrm{pH}$ and salt content of the buffer. Solid-phase purification is usually performed by a use of a spin column, operated by centrifugal force [51]. This method can purify nucleic acid quickly compared to conventional methods. Silica matrices, glass particles, diatomaceous earth, magnetic beads and anion-exchange carriers are examples that have been used as solid support in the solid-phase extraction method. Four key steps involved in solid-phase extraction are cell lysis, nucleic acid adsorption, washing, and elution [49].

The initial step in the solid phase extraction process is to condition the column for adsorption of the sample. Column conditioning can be done by using a buffer at a particular $\mathrm{pH}$ to convert the surface or functional groups to a particular chemical form [51]. Next, the sample which has been degraded by using a lysis buffer is applied to the column. The desired nucleic acid is absorbed into the column by means of high $\mathrm{pH}$ and salt concentration of the binding solution [51]. Other compounds, such as protein may also have a strong specific bond to the surface of the column. These contaminants can be removed using a buffer containing a competitive agent during the washing process [51]. For the elution step, the TE 
buffer or water is introduced to release the desired nucleic acid from the column, so that it can be collected in the purified state [51]. Rapid centrifugation, vacuum filtration or column separation is normally required during the washing and elution phases of the purification process.

\subsubsection{Magnetic Bead based Nucleic Acid Purification}

Magnetic separation is a simple and effective method that nowadays is used in nucleic acid purification. Many magnetic carriers are commercially available. Particles with a magnetic charge may be removed by using a permanent magnet when applying a magnetic field. Magnetic carriers with immobilised affinity ligands or prepared from a biopolymer showing affinity to the target nucleic acid are often used in isolation. Magnetic particles, for example, that are produced from different synthetic polymers, biopolymers, porous glass or magnetic particles based on inorganic magnetic materials such as surface-modified iron oxide. Materials with a large surface area are preferred for use in the binding of nucleic acids. Magnetic particulate materials such as beads are more preferable to isolation support due to their higher binding capacity. The nucleic acid binding process may be assisted by the nucleic acid "wrapping around" the support. A magnet can be applied to the side of the vessel, which contains the sample mixture for aggregating the particles near the wall of the vessel and pouring out the remainder of the sample [52].

Particles with magnetic or paramagnetic properties are used in an invention where they are encapsulated in polymers such as magnetizable cellulose [53]. Magnetizable cellulose may bind to nucleic acids in the presence of certain concentrations of salt and polyalkylene glycol. Small nucleic acid required higher concentrations of salt to be strongly bound to the magnetizable cellulose particles. Therefore, salt concentration can be selectively manipulated to release nucleic acid bound to magnetizable cellulose by size. Magnetizable cellulose to nucleic acid shall be washed with an appropriate wash-buffer before contact with an appropriate elution buffer to separate the desired nucleic acid from cellulose. Separation of 
magnetizable cellulose from the supernatant during all the purification steps can be done by applying a magnetic field to draw down or draw them to the side of the vessel [53]. The magnetizable cellulose used in this invention has a content of iron oxide up to about $90 \%$ by weight of the cellulose total mass. Other magnetic compounds, such as ferrous oxide or nickel oxide can also replace the magnetic component of cellulose [53].

An extraction kit based on the principle of nucleic acid purification which uses magnetic beads is commercially available on the market [54]. The special part of this kit is that the reagents supplied are intended for use with magnetic tools. This magnetic tool is recommended when working in a microtube format. It is a practical device for performing separations based on the technology of magnetic particles. The kit does not require any organic solvent and eliminates the need for repeated centrifugation, vacuum filtration or column separation. The protocol is based on a modified alkaline lysing procedure followed by the binding of nucleic acid to magnetic particles. The magnetic tool is used to capture magnetic particles with bound nucleic acid and the contaminants are removed by washing with the provided wash buffer. Nucleic acid is then removed from the magnetic particles by means of an elution buffer [54].

\subsection{Automated Extraction System}

Promega Corporation [55] has introduced an automated extraction system, a large, expensive and complex instrumentation designed for high-throughput sample processing, which has helped to simplify nucleic acid insulation. An additional example of an automated system that is flexible and efficient in the extraction of nucleic acids and proteins has been introduced by Analytik Jena AG [56]. Boyd [57] discussed that the automated process of extracting nucleic acid is potentially beneficial for a number of reasons, including a reduction in working time, a reduction in labour costs, an increase in the safety of workers and an increase in 
reproducibility and quality of results. It is also a key solution for increasing the laboratory efficiency [55]. The speed, accuracy and reliability of the entire extraction process should be maximum and at the same time minimise the risk of cross-contamination as discussed by Loeffler et al. [58]. Although automation has played a key role in increasing the throughput and improving process reliability, the tools are still designed solely for use in the laboratory environment. An environmental DNA sample must be sent to the laboratory for analysis. This is not always feasible and may be inconvenient, particularly in the case of astrobiological exploration. Haeberle et al. [59] presented an automated nucleic acid extraction unit in which a Coriolis force acutated droplet router was used to route the fluid. However, the discussion on the upstream and downstream processes has not been clearly discussed.

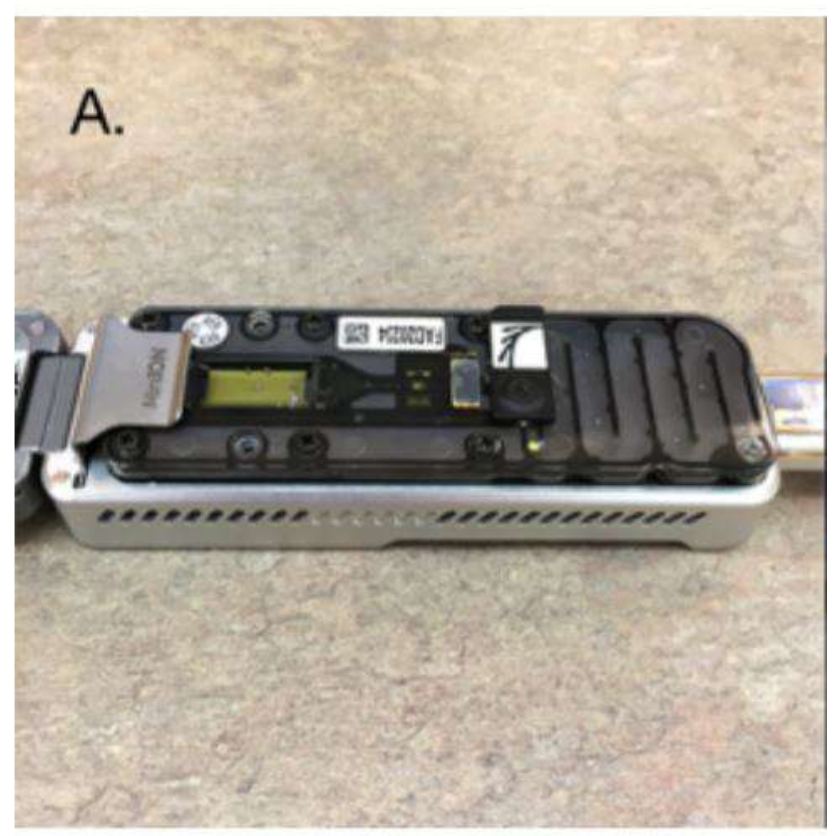

Figure 2.3: MinION Mk 1B with R9 flowcell [60].

Mojarro et al. [60] are developing an integrated nucleic acid extraction and sequencing instrument: the Search for Extraterrestrial Genomes (SETG) for in situ life detection at Mars. The automated extraction module is a customised Claremont BioSolutions SimplePrep X1 Automated Lysis and Nucleic Acid Extraction platform. 


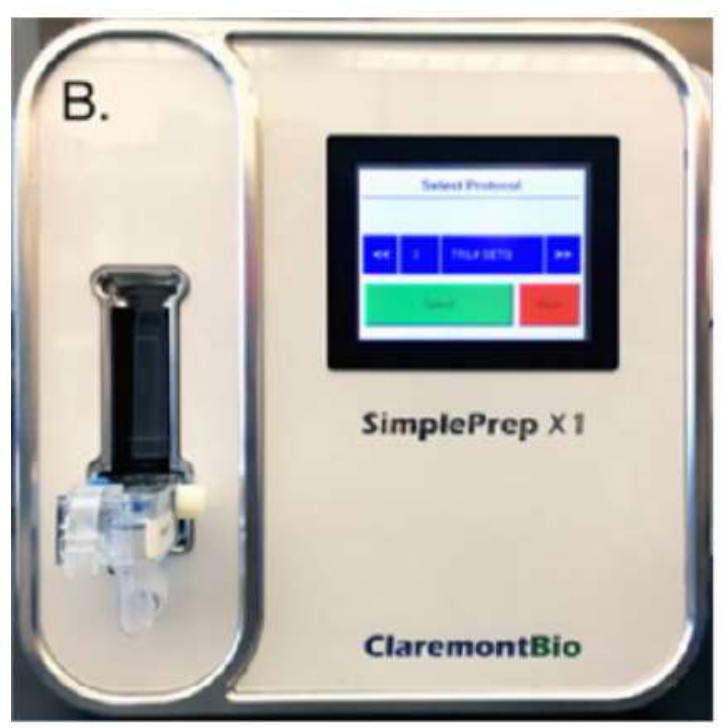

Figure 2.4: Claremont SimplePrep X1 automated lysis and nucleic acid extraction platform [60].

\subsubsection{Microfluidic Approach}

The enormous interest in microfluidics has been stimulated in part by the realization that macroscale properties, such as laminar flow, small diffusion distance, high surface area to volume ratio, and electroosmotic flow potential can be exploited to yield improved performance for analytical methods [61][62]. Another important factor driving the development of microfluidics has been the use of microfabrication methods based on common semiconductor processing techniques. These methods have made it possible to develop complex fluid circuits that integrate multiple analytical and sample processing functions, such as sampling, filtering, derivatization, dilution, and separation into a monolithic device [63-69]. This integration of functions results in highly automated and rapid measurements even when considerable complexity is required. Due to the small size of the channels used, low volumes (nanoliters and less) are manipulated, resulting in reduced reagent consumption and easier analysis of microscale samples. Microfabrication also facilitates the development of parallel architectures for analytical measurements with a potential for high-throughput analysis. Automated microfluidic devices have proved capable of performing thousands of chemical assays per day [70][71] and could potentially 
perform millions of assays per day in the near future.

For example, a rapid single cell or cell-group analysis can be performed on microfluidic chips to provide an efficient pathway for the determination of statistical variation between biological entities [71][72]. Such methods benefit from the integration of fluid function, miniaturisation of sample size, improved analytical properties and high throughput capability. Since microfluidic devices can be manufactured from a number of low-cost materials, disposable devices [73][74] can also be developed. Although enormous progress has been made in developing fluid manifolds or networks capable of processing samples on chips, in some cases chips smaller than the US or Canadian dime [74], almost all chips reported to date require external auxiliary equipment such as pumps, valves, detectors and power supplies [75]. This auxiliary equipment makes these devices far from portable, small, and low-power "lab-on-a-chip" ideal. However, progress has been made towards fully integrated or at least partially integrated and highly portable devices. For example, [76][77] have described portable microfluidic platforms used as biosentinels for space exploration. In addition, commercial manufacturers have made great strides in developing key components such as low-current power supplies, lasers, LEDs and pumps that are more suitable for portable microfluidic platforms.

The integrated genetic analysis system was also demonstrated by Easley et al. [78]. This device has a number of integrated sample handling components including: nucleic acid purification by solid phase extraction, PCR by thermal cycle and elastomeric valves, and electrophoretic separation. The off-chip components of this device include LIF detection components, pressure-driven fluid flow syringe pumps, IR thermal cycler and control / power systems. This device was capable of performing a total DNA analysis in less than 24 min with a migration separation of less than 180 seconds. The authors speculate that their device may have a detection limit on the order of a few hundred starting copies of DNA. Pal et al. [74] have developed an integrated microfluidic device capable of genetic analysis of 
viral DNA (e.g. influenza). This microfluidic device integrates fluid manipulation, thermal heaters, temperature sensors, valves and electrophoretic separation. The off-chip components include pneumatic control, epi-confocal illumination, ICCD camera, and power/control systems. Separations of PCR products were carried out in less than $140 \mathrm{sec}$, while 35 thermal cycles were performed in less than 20 min. This device is manufactured from a silicone substrate that could provide future advancements, including the integration of LIF optics and CMOS control units [74].

Although GC-MS coupled with pyrolysis extraction has been the instrument of choice for past flight missions and has a legacy of space flight operations, this technique has poor quantitative capabilities and is generally less sensitive than LIF detection. In addition, there is considerable evidence that pyrolysis disrupts the original composition of the sample due to unpredictable chemical processes that may occur during heating and can also lead to a loss of volatiles [79-81]. Irrespective of the technique chosen, the in situ analysis of complex unknown mixtures is challenging and may require a wide range of experimental conditions in order to perform an exhaustive chemical analysis. In the case of GC-MS, several separation columns for each type of compound must be used for this purpose. Conversely, a microfluidic device can perform a complete analysis by performing a pre-programmed sequence of experiments with a variety of different separation conditions using a single channel.

\subsection{Summary}

Nanopore based sequencing technology was discussed in this chapter. An introduction to the types and challenges associated with nanopore sequencing technology has been provided. A simple yet efficient way of purifying the nucleic acid was discussed along with the test procedure. In this thesis, nanopore based sequencing is used for DNA / RNA sequencing and $\mu$-MAMA is used for the detection 
of microbial activity. A microfluidic approach, together with the use of external pumps, valves and tubes to drive the fluid, is used to integrate and automate multiple instrumentation suites.

The following chapter discusses the mathematical model and methodology used to integrate the instrumentation unit. 


\section{Chapter 3}

\section{Automation Methodology}

In order to properly understand the behaviour of the fluid and methodology of this thesis, it is important to provide some information on the mathematical model used for the determination of flow-related parameters. A detailed explanation of the mathematical model and phase types that exist in the microfluidic system is discussed in section 3.1. Section 3.2 outlines the methodology used to automate the instrumentation suite. A brief discussion on the material to be used for the production of microchip is presented in section 3.3. Section 3.4 describes the components used to develop the instrumentation suite. The design process of the novel microbial activity micro assay is detailed in section 3.5. Device manufacturing and the study of different microfluidic chip models for automated nucleic acid extraction system are presented in section 3.6. Finally, the 3.7 section provides a summary of the work performed in this chapter.

\subsection{Mathematical Model of Microfluidic System}

To understand the flow of fluid through the circular cross-section, the fluid is assumed to be incompressible and Newtonian, the flow is laminar, and the circular cross-section of the tube is assumed to be substantially longer than its diameter. Also, there is no relative motion between the fluid particles and the boundary layer, i.e. no slip of fluid at the boundary layer. 


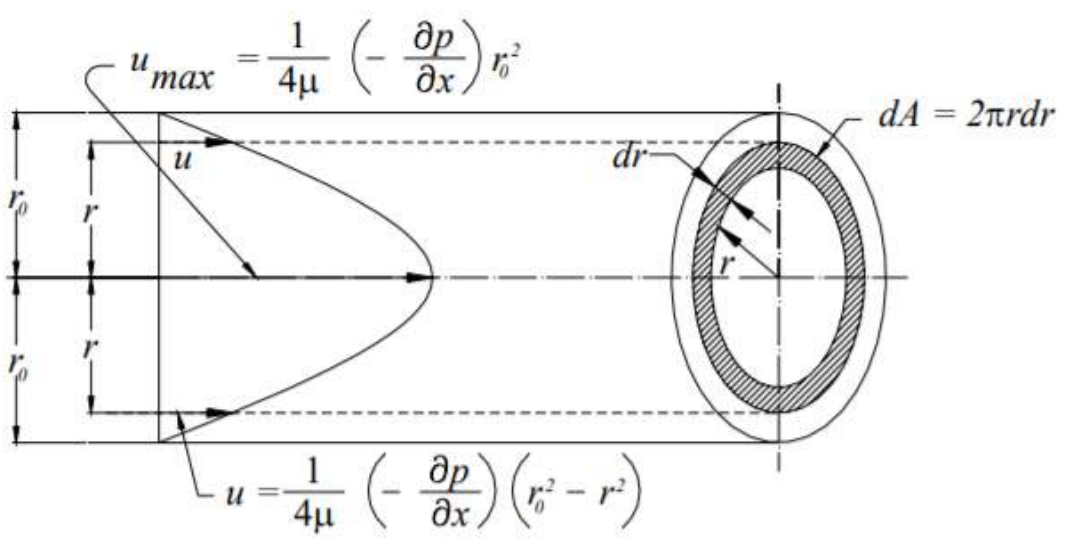

Figure 3.1: Laminar flow in a straight circular tube with radius [82].

\subsubsection{Continuous Phase}

In a droplet-based microfluidic network, a fluid called a continuous phase, with a dynamic viscosity of $\mu_{c}[\mathrm{Pas}]$, is injected into the channels by an external source, in this case a peristaltic pump that would impose a pressure gradient of $\Delta P[\mathrm{~Pa}]$ across the channel. The average velocity of the fluid through the channel is $\frac{Q_{c}}{A}$, or

$$
V=\frac{Q_{c}}{\pi r_{0}^{2}}=\frac{1}{8 \mu_{c}}\left(-\frac{\partial p}{\partial x}\right) r_{0}^{2}
$$

where, $r_{0}^{2}$ is the radius of the circular cross-section and $Q_{c}$ is the volumetric flow rate given by,

$$
Q_{c}=\frac{\pi}{8 \mu_{c}}\left(-\frac{\partial p}{\partial x}\right) r_{0}^{4}
$$

Pressure gradient $\frac{\partial p}{\partial x}$ in the direction of flow depends on $x$ only for a given case of laminar flow. A negative sign indicates a decrease in fluid pressure in the direction of flow in the horizontal tube, since the work of flow must be performed to compensate for the frictional resistance to flow. Now the pressure drop between $p_{1}$ and $p_{2}$ for any stretch of the tube between $x_{1}$ and $x_{2}, L=x_{2}-x_{1}$ is given by,

$$
\int_{p_{1}}^{p_{2}} \partial p=\frac{8 \mu_{c} V}{r_{0}^{2}} \int_{x_{1}}^{x_{2}} \partial x
$$


and since diameter $D$ equals $2 r_{0}$

$$
\Delta P=\frac{8 \mu_{c} V L}{r_{0}^{2}}=\frac{32 \mu_{c} V L}{D^{2}}
$$

where presure drop $\Delta P=p_{1}-p_{2}$. Equation (3.4) is usually referred to as the Hagen-Poiseuilli equation. To calculate the volumetric flow rate $Q_{c}$, in the microfluidic channel of the length $L$ is given by the law,

$$
R_{h}=\frac{\Delta P}{Q_{c}}
$$

Here, $R_{h}$ is the microfluidic flow resistance that quantifies the energy drop along the microfluidic channels. Just as the electrical potential drop $d V$ is proportional to intensity $A$, fluid pressure drop $d P$ is proportional to the mean flow rate $Q_{c}$ and resistance $R_{h}$ and can be defined as the proportional coefficient. Thus the microfluidic resistance $R_{h}$, across a simple cicular cross-section is given by the following equation,

$$
R_{h}=\frac{128 \mu_{c} L}{\pi D^{4}}
$$

From the above equation, we can say that factors that must affect microfluidic resistance depend on the following:

1. Cross-section area and length of the tube

2. Type of fluid flowing through the tube and

3. Inside finishing of the tube

In addition, longer channels with smaller cross-section area would have higher microfluidic resistance and vice versa. Fluids with lower dynamic viscosity would have lower resistance from the walls and can flow freely without any disruption. 


\subsubsection{Dispersed Phase}

In a droplet-based microfluidic network, a second fluid called dispersed phase and with a dynamic viscosity $\mu_{d}$, is injected into the continuous phase by an independent pump, which in this case is a peristaltic pump. The interfacial tension coefficient between the dispersed phase and the continuous phase, denoted by $\sigma$ $[\mathrm{N} / \mathrm{m}]$, determines the value of the capillary number $C_{a}$, a dimensionless parameter that describes the relative magnitude of the viscous shear stress compared to the interfacial tension, given by,

$$
C_{a}=\frac{\mu_{c} u_{c}}{\sigma}=\frac{\mu_{c} Q_{c}}{\sigma \pi r_{0}^{2}}
$$

For the dispersed phase to form droplets of regular size, the system shall work in the so-called squeezing regime [83][84], which requires

$$
C_{a} \leq C_{a}^{*} \approx 10^{-2}
$$

This limit, in turn sets an upper bound $u_{c}^{*}$ to the continuous phase speed $u_{c}$ that is,

$$
u_{c} \leq u_{c}^{*}=\frac{\sigma}{\mu_{c}} 10^{-2}
$$

In this research, it was assumed that equation (3.9) is satisfied and that the dispersed phase takes the form of regular droplets, whose length $l_{d}$ can be approximated as,

$$
l_{d}=r_{0}\left(1+\varepsilon \frac{Q_{d}}{Q_{c}}\right)
$$

where, $\varepsilon$ is the dimensionless parameter of the order of 1 that depends on the absolute value of $Q_{c}$. From mass conservation principle, it follows that the inter- 
droplet distance is given by,

$$
\delta=l_{d} \frac{Q_{c}}{Q_{d}}=r_{0}\left(\frac{Q_{c}}{Q_{d}}+\varepsilon\right)
$$

The droplet length per inter-distance can then be tuned by adjusting the volumetric flow rates $Q_{c}$ and $Q_{d}$ of the continuous and the dispersed phases respectively, if equation (3.9) holds true. When a droplet is injected into the cross-section, the friction generated with the carrier fluid and the forces produced by the inhomogeneity between the dynamic viscosity of the continuous and the dispersed phases determine a change of the hydraulic resistance of the channel [85-87]. Mathematically the variation of resistance produced by a droplet of length $l_{d}$ in a channel of length $L$ is given by [88],

$$
\begin{aligned}
\rho\left(l_{d}\right) & =R\left(\mu_{d}, l_{d}\right)-R\left(\mu_{c}, l_{d}\right) \\
& =\frac{128 \mu_{d} l_{d}}{16 \pi r_{0}^{4}}-\frac{128 \mu_{c} l_{d}}{16 \pi r_{0}^{4}} \\
& =\frac{8 l_{d}}{\pi r_{0}^{4}}\left(\mu_{d}-\mu_{c}\right)
\end{aligned}
$$

such that the overall microfluidic resistance of the channel when crossed by a droplet of length $l_{d}$ is the $R\left(\mu_{c}, L\right)+\rho\left(l_{d}\right)$, where $R\left(\mu_{c}, L\right)$ is a function of dynamic viscosity of the dispersed phase and length of the tube. Note that these relationships are only true when the droplet is large enough to fully fill the channel section, with an extremely thin stream of continuous phase between the droplet and the channel surfaces. In this case, when crossing a channel, the speed of the droplet is essentially the same as that of the continuous phase in that channel, i.e., where $Q_{c}$ is the volumetric flow rate of the continuous phase in the channel, and $\Delta P$ is the pressure gradient between the endpoints of the channel. As a result, the microfluidic resistance in the different parts of the network may change over 
time, depending on the path followed by the droplets and their size.

$$
\begin{aligned}
u_{d} & =u_{c}=\frac{Q_{c}}{\pi r_{0}^{2}} \\
& =\frac{\Delta P}{\pi r_{0}^{2}\left(R\left(\mu_{c}, L\right)+\rho\left(l_{d}\right)\right)} \\
& =\frac{\Delta P}{\pi r_{0}^{2}\left(\frac{8 \mu_{c} L}{\pi r_{0}^{4}}+\frac{8 l_{d}}{\pi r_{0}^{4}}\left(\mu_{d}-\mu_{c}\right)\right)} \\
u_{d} & =\frac{\Delta P}{\frac{8}{r_{0}^{2}}\left(\mu_{d} l_{d}+\mu_{c}\left(L-l_{d}\right)\right)}
\end{aligned}
$$

However, the speed of the droplets depends on the volumetric flow rate of the continuous phase in the different branches, which, in turn, depends on the microfluidic resistance of the channels and the position of the droplet. Thus from the above mentioned equations, diameter of the tubing ideal for flow automation and the flow rate needed to transfer the fluid from one section to another was calculated which was later used to identify and select off-the shelf components such as peristaltic pumps and tubing.

The following sections discuss the automation methodologies and instrumentation requirements that implement the results of the mathematical models mentioned in the previous section.

\subsection{Methodology for Automation of the Device}

Instruments used for planetary exploration and biochemical analysis should perform experiments with extreme precision and sensitivity so as not to contaminate the sample while providing high throughput results. On the other hand, the designed micro-life system should be compact enough to reduce the overall mass of the instrument suite. For example, the Curiosity rover (launched in March 2011) included the Sample Analysis at Mars (SAM) instrument suite containing the GC-MS (ppb reported sensitivity) and the tunable laser spectrometer (LOD $2 \mathrm{ppb}$ for methane and $2 \mathrm{ppm}$ for water). This suite weighed approximately 38 $\mathrm{kg}$ and accounted for $63 \%$ of the volume of the entire analytical laboratory $(53 \times$ 
$42 \times 31 \mathrm{~cm})$. On the other hand, the instrumentation suite described here weighs around $6 \mathrm{~kg}$ and $35 \times 31 \times 17 \mathrm{~cm}$, which makes it $\sim 6$ times lighter and five times smaller than the current technology used for extraterrestrial exploration.

The designed micro-life subsystem consists of a peristaltic micro-pump, which was the driving mechanism of the fluid from the heating cup to the filtration chamber. It also consisted of a heated cup, which is a closely packed model and resembles a bottle of a coaster. They use a ceramic heater coil and, once connected to the circuitry, the electricity flows and heats up the heating element. This element plays an essential role in the event that the sample collected is completely frozen and needs to be analysed in a liquid state. The micropump used to transfer the fluid maintained a controlled flow rate with high accuracy and repeatability. A circuit diagram schematic is shown in figure (3.2).

\subsubsection{Instrumentation Requirements}

In addition to high precision and accuracy, the pump should be smaller in size in order to reduce the overall mass of the system. The micropump chosen for the subsystem was available off the shelf. Since a low flow rate micropump is required in microfluidics (with pump flow rate in microliter per minute, $\mu \mathrm{l} / \mathrm{min}$ or sometimes nanoliter per minute, $n l / \min$ ), the pump chosen had a maximum flow rate of approximately $40-60 \mu l / m i n$. In addition, the tube chosen to transfer the fluid from one section to another should not contaminate the sample to be processed. Transparent polytetrafluoroethylene (PTFE) coated tubing, commonly referred to as Teflon tubing, was therefore chosen for this application. These tubes are highly flexible, chemically inert, thermally stable enough to be used without degradation between $-200^{\circ} \mathrm{C}$ and $260^{\circ} \mathrm{C}$.

Other useful properties include high flexiural strength, low temperatures, high electrical resistance and dielectric strength, water resistance (due to high electronegativity of fluorine) and low friction coefficient. PTFE's density is also very high, at $2200 \mathrm{~kg} / \mathrm{m}^{3}$. However, the only factor to be taken into account when 


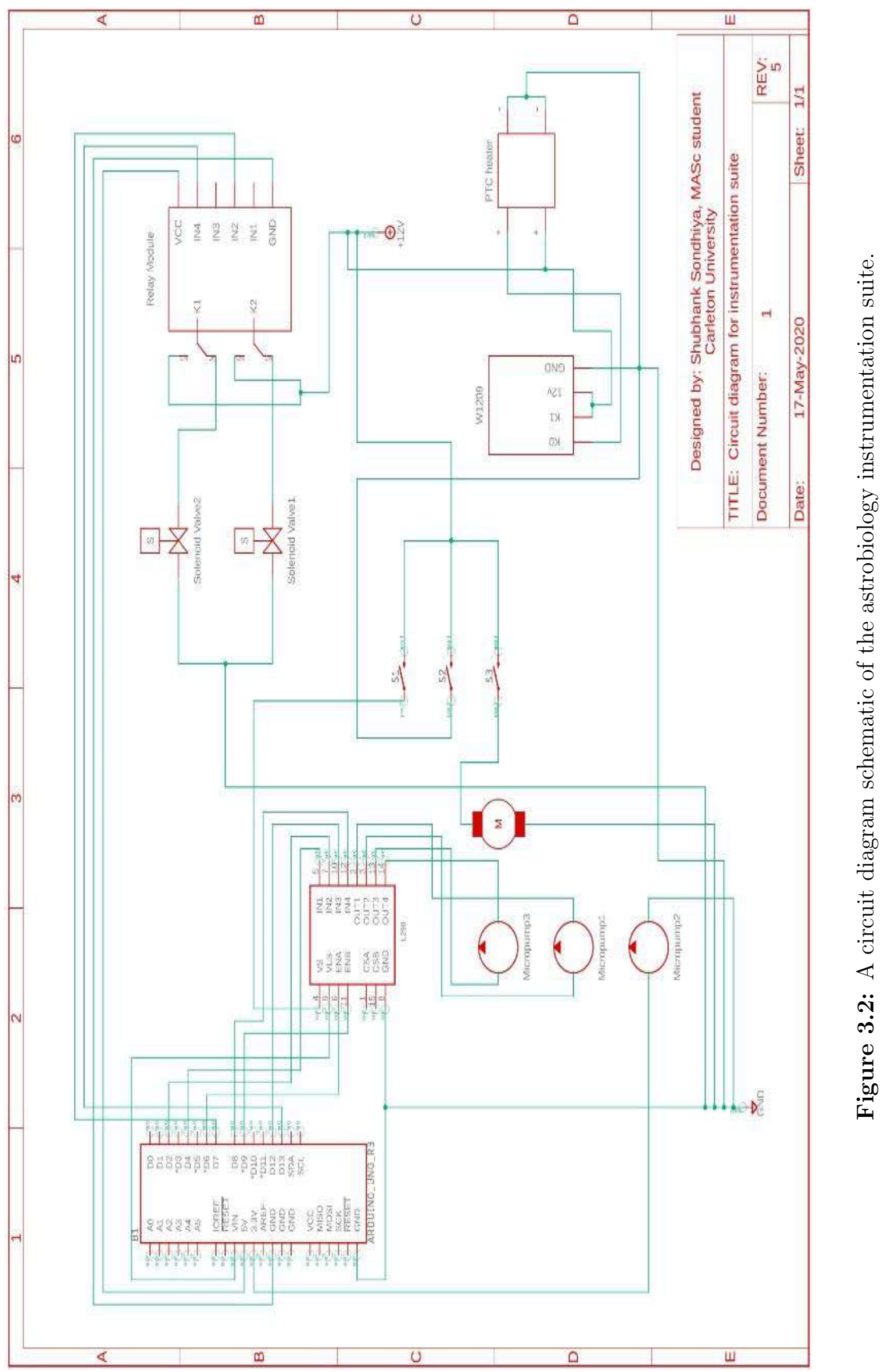


using PTFE is that it does not have a good resistance to high-energy radiation, which causes the PTFE molecule to break down.

Table 3.1: Micropump parameters and their specifications.

\begin{tabular}{|c|c|}
\hline Parameters & Specification \\
\hline Flow rate & $0.1 \mu \mathrm{l} / \mathrm{min}-40 \mu \mathrm{l} / \mathrm{min} \pm 15 \%\left(\right.$ sample at $\left.25^{\circ} \mathrm{C}\right)$ \\
\hline Tubing material & PTFE coated silicone tubes \\
\hline Pump pressure & with an inner diameter of $0.5 \mathrm{~mm}$ \\
\hline Motor control & 30 KPa or more \\
\hline Dimensions & Stepper motor controlled \\
\hline Pump head & $33 \mathrm{~mm} \times 12 \mathrm{~mm} \times 21.5 \mathrm{~mm}$ \\
\hline
\end{tabular}

Due to its high flexibility and other such advantages, it made a clear choice for this application. In order to ensure that the pump chosen meets the standards, certain key points have been taken into consideration which are as follows;

1. Compact size to reduce the overall mass of the subsystem,

2. Low to medium pressure range (30Kpa or more),

3. Stepper motor controlled to increase the accuracy when transferring the fluid, and

4. Low power consumption and a replaceable pump head, in case design needs to be modified to meet the requirements.

The RP-TX series micropump manufactured by Takasago Electric INC. [89] as shown in the figure (3.4) met the above requirements and the specifications are shown in the table (3.1). The ATmega328P Arduino Uno micro-controller was used to control the flow rate of the pump. One of the chambers in the microlife system included the AlamarBlue reagent. It is ready to use a resazurin-based 
solution that acts as an indicator of cell health by using the reduced power of living cells to quantify viability. The AlamarBlue reagent is non-toxic, a cell-permeable compound of blue colour and virtually non-fluorescent. Upon entry into the living cells, resazurin reduces to resorufin, a red compound that fluoresces strongly. Due to its wide applicability, it can be used with various cell lines, bacteria and fungi. Outstanding features include:

1. Robust and reliable performance that results in a large, highly reproducible dynamic range,

2. Highly sensitive reagent with a linear response as it detects as few as 50 cells per well,

3. Convenient add-and-read format, that is, no mixing, no washing, no cell lysis

4. Compatible with either fluorescence- or absorbance-based instrumentation, and

5. Measures viability from many diverse cell types including mammalian cells, bacteria, plant, and fungi.

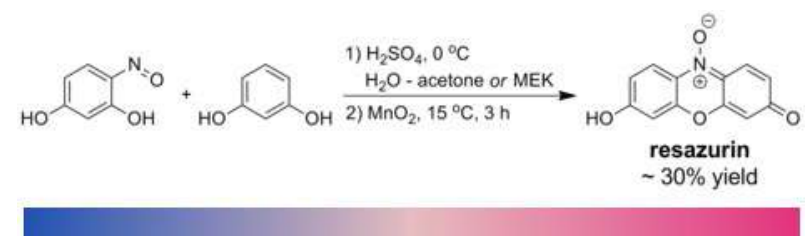

Figure 3.3: Chemical formula for alamarBlue dye.

In order to protect the reagents and sample from contamination, choosing the correct tubes was also maintained at high priority. Falcon ${ }^{\mathrm{TM}}[90]$ conical tubes were preferred as they are easy to use and of high quality to protect the samples. The BD Falcon tubes are made of high quality polypropylene. BD falcon $50 \mathrm{ml}$ conical tubes with a screw-top cap were used in this study. The state-of-the-art design and manufacturing provides high strength, wide temperature stability for experimenting in extreme environments. In addition, these tubes are biocompatible as during design resins are selected via an intense array of U.S. Pharmacopoeia 
(USP) toxicity tests to ensure the products do not contain concentrations of bioactive substances that could potentially interfere with biological experiments.

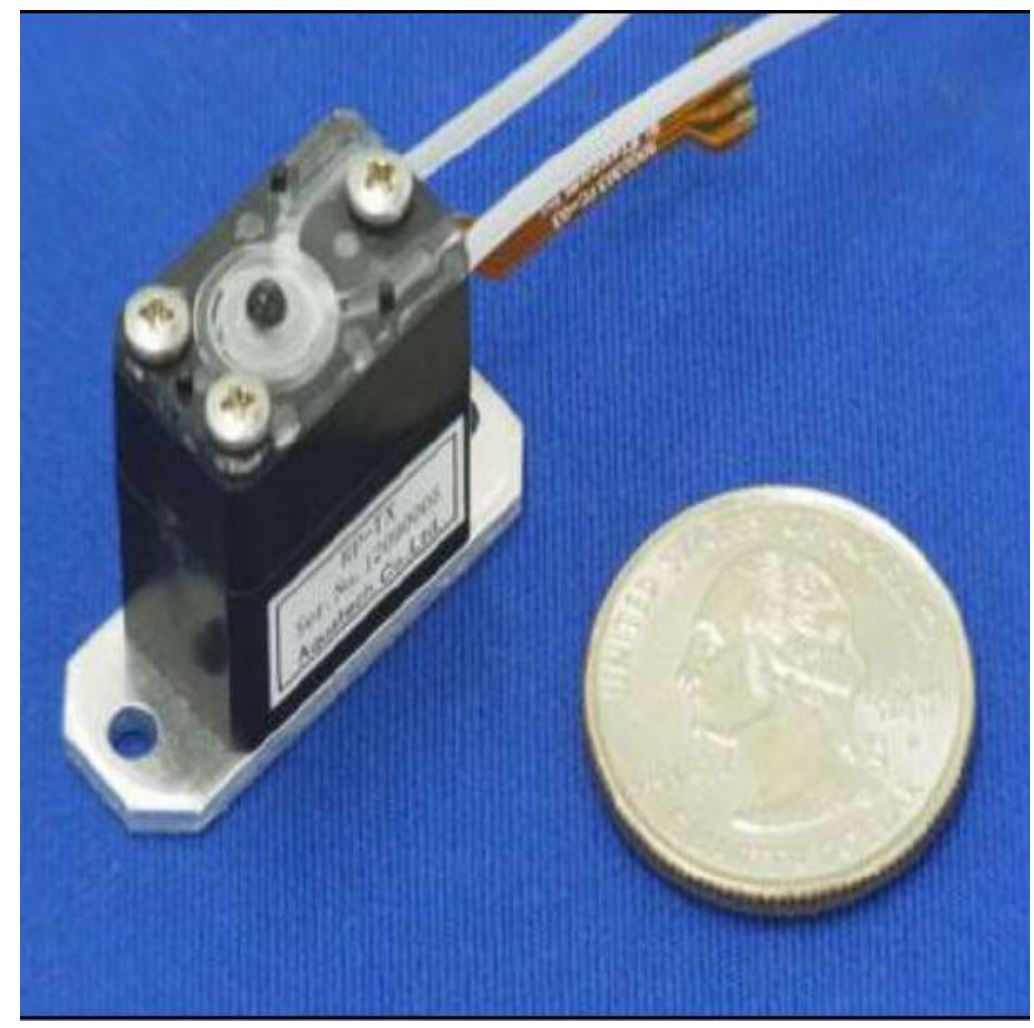

Figure 3.4: Image of an RP-TX series micropump used in the micro-life system; scale: $33 \mathrm{~mm} \times 12 \mathrm{~mm} \times 21.5 \mathrm{~mm}$ [89].

\subsection{Fluidic Manipulation}

In 1990, Manz et al. [62] introduced the concept of integrating sampling, sample treatment, seperation and detection into a single microfluidic device as Miniaturized Total Analysis Systems ( $\mu$-TAS) . Although processes such as enzymatic assays, reactors, mixers, and extractors have been successfully integrated into microfluidic devices for various applications [78][91-95], the focus here was on microchip functionalities specifically targeted for in situ automated measurements. The most commonly used polymer for microfluidic design manufacturing was Polydimethylsiloxane (PDMS) due to its low cost, ease of manufacturing, elasticity and good adherence to glass [76][96][97]. Although PDMS is a viable option for in situ analysis on Mars where microchips are supposed to analyse only aqueous 
soil extracts, its incompatibility with organic solvents limits its applications for space flights to targets such as Enceladus, Europa and Titan requiring organic solvents. Other polymeric materials that are more resistant to organic solvents have been successfully incorporated into layered microchip models, including Teflon ${ }^{\circledR}$ [98][99] and Fluorocur ${ }^{\mathrm{TM}}$ perfluoropolyether (PFPE) [100].

Another factor to be considered when selecting a material is the thermal cycling that the microfluidic device will be exposed to during space flight applications. In this regard, both the PDMS and the PFPE systems maintain functionality following repeated temperature cycle operations [98-100]. Although PDMS is the most elastic of these materials and therefore delivers the highest volume per stroke, pumping rates are the fastest for Teflon ${ }^{\circledR}$ devices due to their rapid response to applied pressure. Because the Youngs modulus of Teflon ${ }^{\circledR}$ is in the order of $10^{3}$ greater than that of PDMS ( 750 KPa) [101], Teflon enables a higher number of pumping cycles per unit that is not possible with PDMS [98]. The optimal polymetric material should therefore be chosen on the basis of planetary environment of interest and the operations required of the microdevice.

\subsection{Automation of the Microfluidic Network}

Two sub units of instrumentation suite were designed, tested and integrated. Each sub-unit is addressed separately to provide a more detailed explanation. Now, to automate the process, components such as solenoid valves, micropumps and membrane filters were used.

\subsubsection{Non-contact Solenoid Valves}

Non-contact on/off solenoid valves were chosen as suitable for contaminationsensitive applications. These valves stop the flow without contacting the process media by pressing down on the outside of the tubing and using electrical signals to automatically start and stop the flow. The valves chosen were normally closed 
unless they were actuated. The actuator was directly mounted to the body of the valve to minimise movement and reduce wear.

\subsubsection{Splitter Manifold}

The splitter manifold as shown in the figure (3.5) made of PolyEther-Ether-Ketone (PEEK) was used as a sample injection unit on 16 independent wells using a wye connector tube from Darwin microfluidics [102]. All ports are designed with 1/428 UNF thread port configuration to fit $1 / 8$ inch outer diameter PTFE tubes. Using this module significantly reduced the complexity of the system, the power consumption and the number of pumps needed to transfer the fluid from one stage to another.

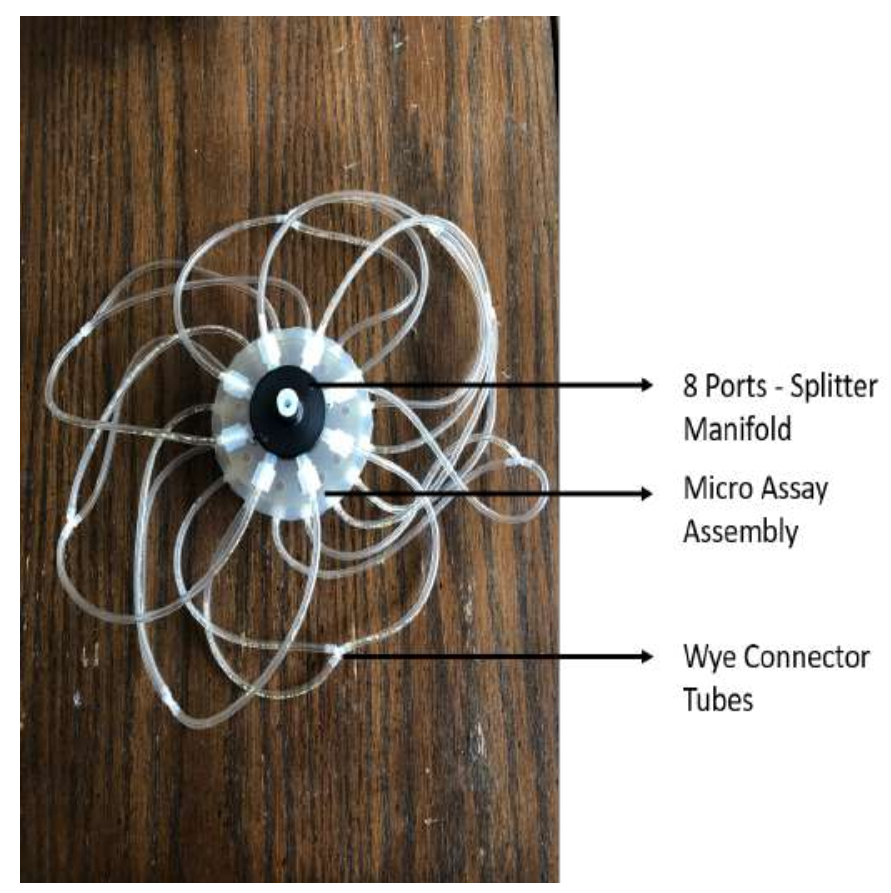

Figure 3.5: 8-Port Splitter manifold with Microassay; scale: $10 \mathrm{~mm}$ (height) $\times$ $35 \mathrm{~mm}$ (diameter).

\subsubsection{Silicone Rubber Gaskets}

To prevent leakage of a sample that could contribute to contamination, highperformance platinum-cured liquid silicone, Dragon Skin ${ }^{\mathrm{TM}} 30$ from Smooth On [103] was used to make transparent sealants that filled the gap between the two 
plates, i.e. the top and base microassay plates. The sealants produced were flexible and resistant to a temperature range of $-53^{\circ} \mathrm{C}$ to $232^{\circ} \mathrm{C}$.

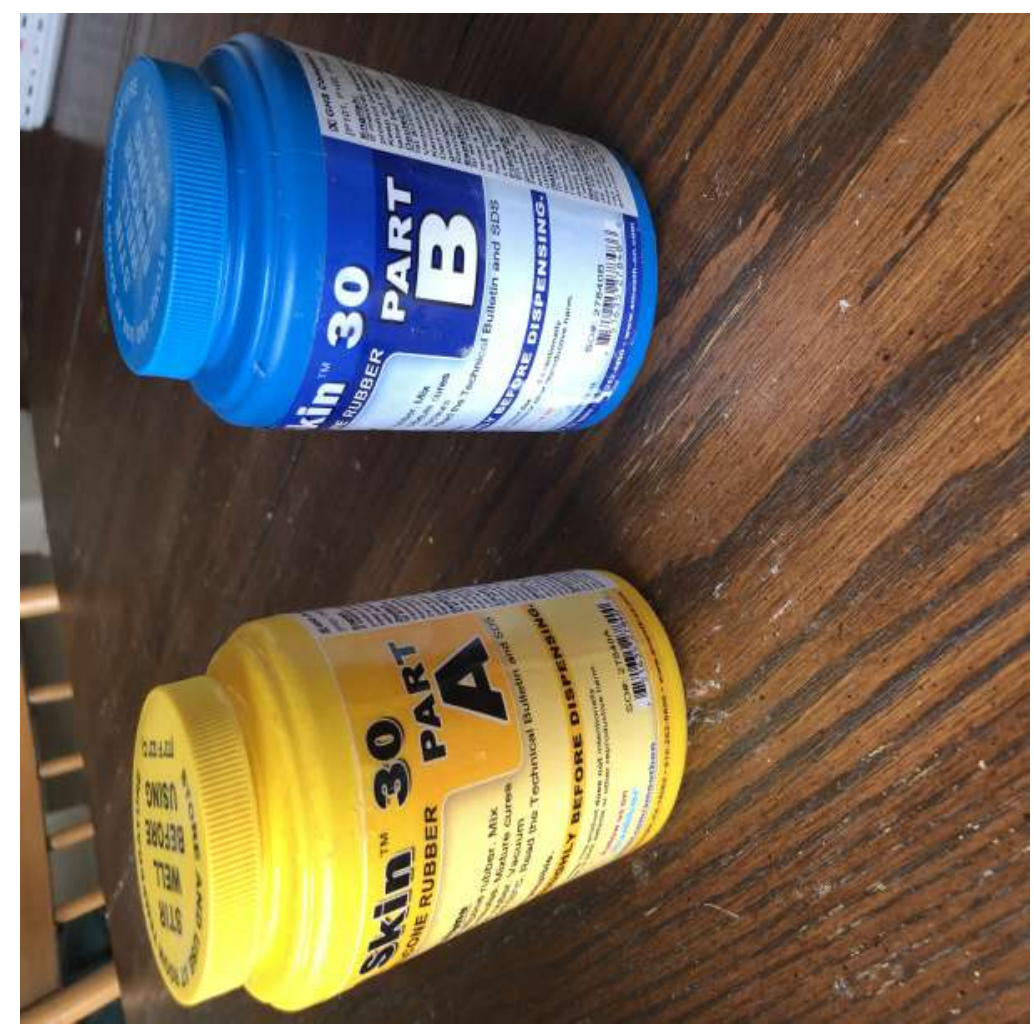

Figure 3.6: Dragon Skin ${ }^{\mathrm{TM}} 30$ high performance platinum cure liquid silicone mixtures.

\subsubsection{Temperature Controlled Chamber}

During field trials, a chamber for in-field incubation of the $\mu$-MAMA sub-unit at the desired temperature $\left(12{ }^{\circ} \mathrm{C}\right)$ was required to check for any microbial activity in the sample collected. To serve this purpose and to preserve the shelf-life of the reagents, a temperature control module was designed as shown in figure 3.7. This module consisted of a ceramic PTC heater, an NTC temperature sensor and a heat sink. The temperature control module W1209 with a thermostat sensor was used to control power and temperature inside the chamber. This module was chosen as the it offers a wide range of temperature control ranging from $-50{ }^{\circ} \mathrm{C}$ to 110 ${ }^{\circ} \mathrm{C}$. It consisted of 3 tactile switches and a built-in micro-controller that allowed different parameters to be configured, including on and off trigger temperatures. 


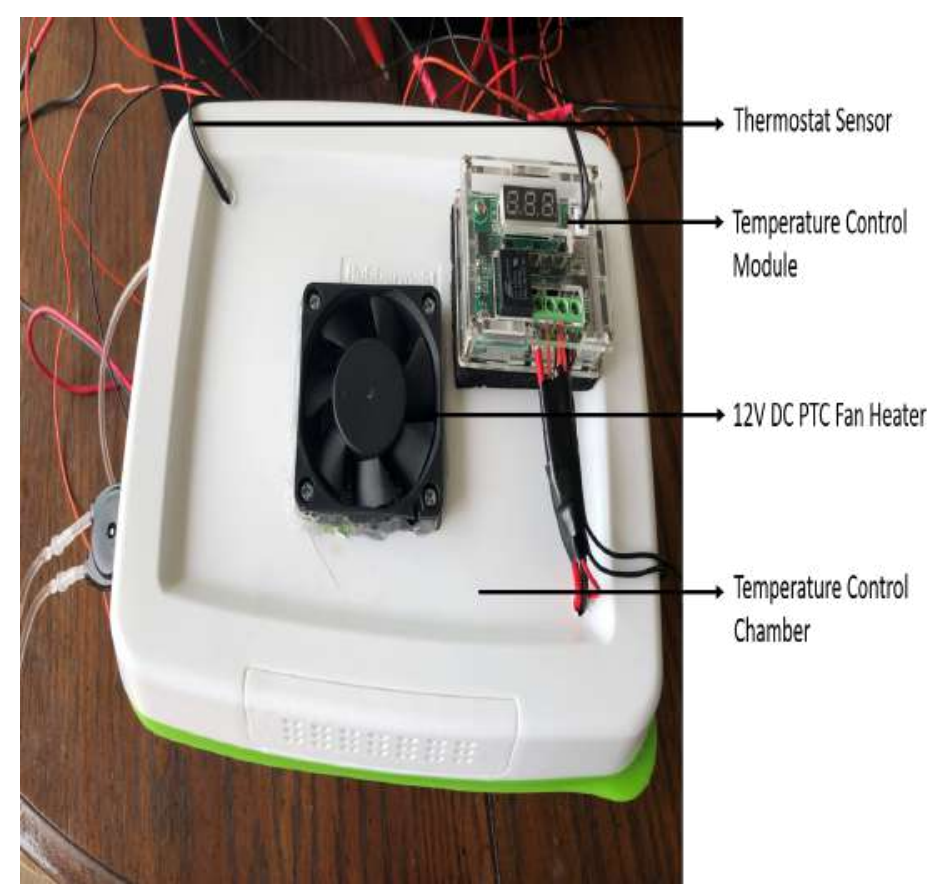

Figure 3.7: Temperature control chamber with temperature control module, a ceramic PTC heater and thermostat sensor; scale: $200 \mathrm{~mm} \times 135 \mathrm{~mm} \times 165 \mathrm{~mm}$.

The onboard relay used can be switched up to a maximum of $240 \mathrm{~V}$ AC at $5 \mathrm{~A}$ or $14 \mathrm{~V}$ DC at $10 \mathrm{~A}$. The current temperature was displayed in degrees Centigrade by means of a 3-digit seven-segment display and the current relay status by an on-board LED.

\subsection{Microbial Activity MicroAssay ( $\mu$-MAMA)}

As redox dye analyses are simple, cost-effective, and highly sensitive to determine and characterise existing microbial activity in situ, they are among the best candidates to be automated for unmistakable microbial metabolic detection.

A Micro Microbial Activity MicroAssay ( $\mu$-MAMA) was developed with its own microfluidic inoculation system capable of detecting and metabolising microbial life in the laboratory and existing microbial life from very low biomass cryoenvironments using redox dye chemistry to detect microbial activity. Redox dyes are reduced, producing a detectable change of colour if the existing microrgansisms in an environmental sample can oxidise the substrate provided (either organic or inorganic). From the experiments carried out by the McGill Research 
team in their laboratory, the BIOLOG Dye Mix G plus the BIOLOG IF-0a buffer and the AlamarBlue dye plus the BIOLOG IF-0a buffer were found to be the best redox dye/inoculation buffer combinations for the following criteria:

1. Detection limits,

2. Robustness, and

\section{Autonomy}

The redox dyes were shown to be very robust as they did not produce false positive redox reactions when exposed to a range of temperatures, pHs, salinity $(\mathrm{NaCl}$, perchlorates), on Mars analog soil. The $\mu$-MAMA design prototype incorporates 16 wells with silicone-sealing gaskets between the top plate and the bottom plate to prevent cross-contamination between wells and the evaporation of the sample. The microbial activity micro-assays are vulnerable to cross-contamination and are available in large sizes. In order to prevent contamination and reduce the size, a 3D printed structure was manufactured as shown in the figure (3.17) with each well having its own inlet and outlet and a sample volume capacity of $100 \mu$ l. During its design process, a few considerations were made:

1. The material used for manufacturing should not hinder/react with the reagents involved in the detection process. In addition, it should be transparent so that the microbial activity could be observed with a naked eye.

2. After curing, the design should be compatible with other instruments.

3. The model should be auto-clavable and bio-compatible.

In this process, three different models were tested, each of which had its own advantages and disadvantages. In order to meet the above requirements, polycarbonate and Veroclear transparent were chosen for 3D printing of the prototype. Both materials had satisfactory efficacy and this design allowed the metabolic activity of a wide range of metabolisms to be taken into account, thus increasing 
the chances of detection. To our knowledge this was the first prototype of the in situ automated detection of metabolic activity.

\subsubsection{First Design}

The design was a square-shaped model with 16 wells in a linear pattern at the same distance relative to each other in such a way that it formed an array of $4 \times 4$ wells. The current microassay and the second model were printed with PolyCarbonate (PC) using SLA 3D printer at McGill University. Four groups, each of which had 4 wells, were made and connected axially with its own inlet and outlet as shown in figure (3.8). In order to prevent reagent leaching or sample loss

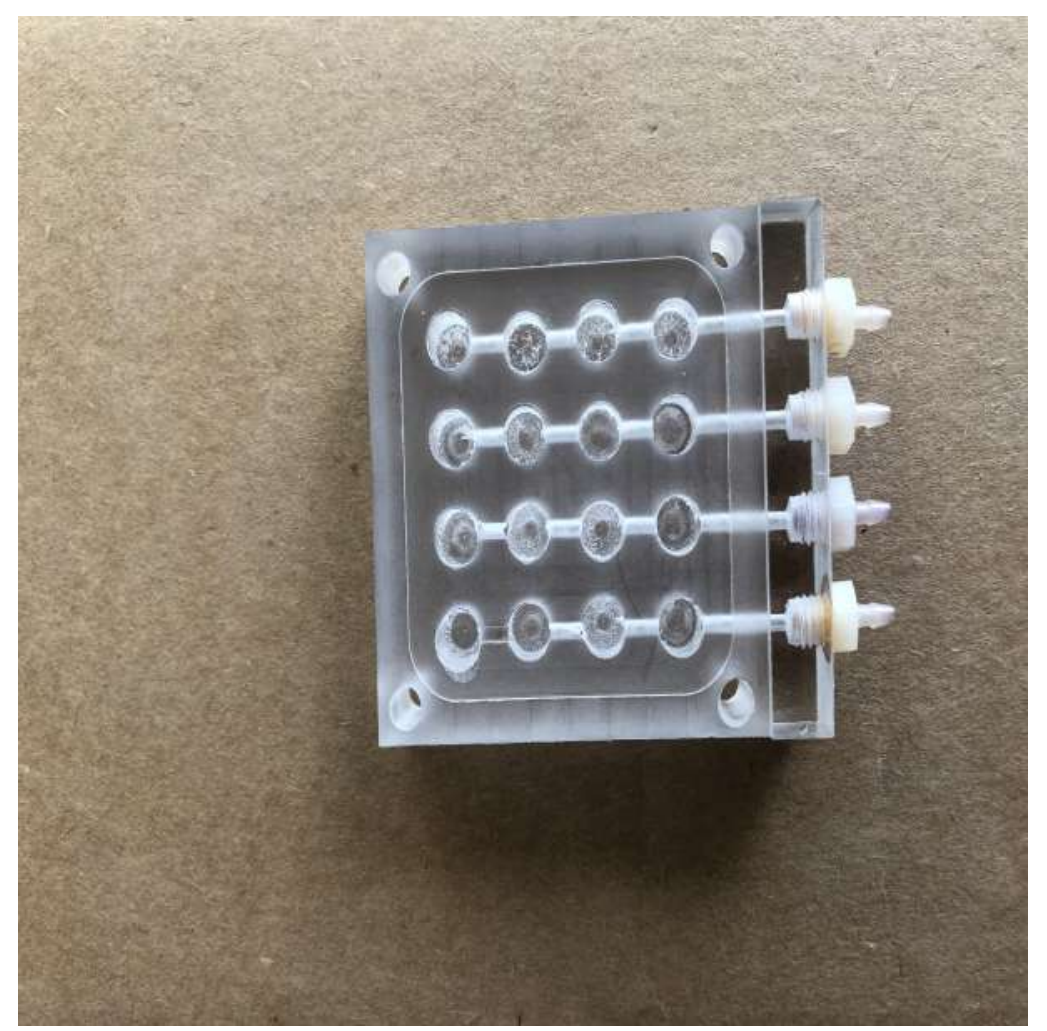

Figure 3.8: Base plate for the first $\mu$-MAMA model with tight seal barbed fittings; scale: $46 \mathrm{~mm} \times 46 \mathrm{~mm} \times 5 \mathrm{~mm}$.

and increase reliability, 10-32 UNF internal threads were made to fit the tight seal barbed fittings. These fittings had a single barb that produced a smooth clamping surface for extra-tight connections with temperatures ranging from $-40^{\circ} \mathrm{C}$ to $135^{\circ}$ C. The other end of the barbed fittings was compatible with the 1/16 inch outer 
diameter of the Teflon tube chosen for this application. The microassay was sealed using the top plate as shown in the figure (3.9) and the silicone gasket (see figure (3.10) to prevent contamination.

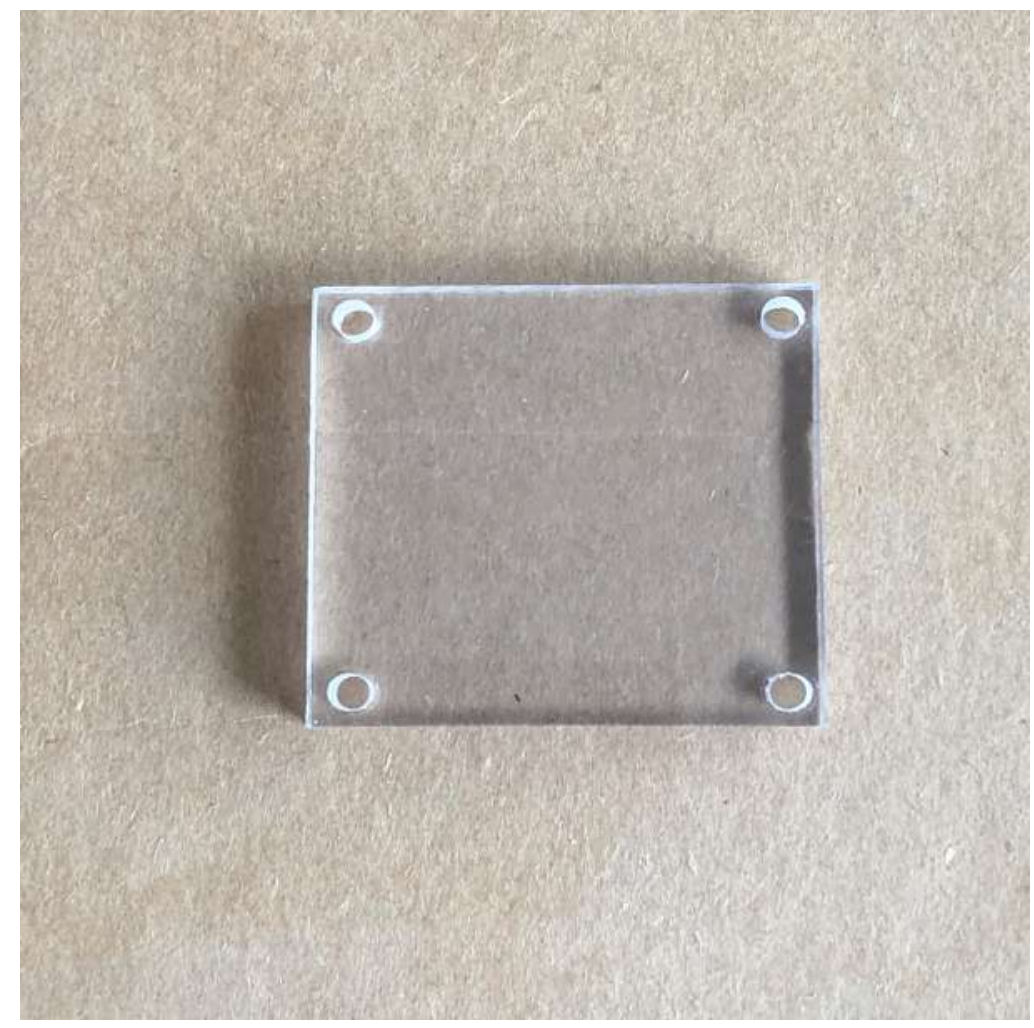

Figure 3.9: Top plate for the first $\mu$-MAMA model; scale: $56 \mathrm{~mm} \times 46 \mathrm{~mm} \times$ $10 \mathrm{~mm}$.

After field tests with the current design, it was observed that it became difficult to analyse multiple environmental sample as 4 wells were connected to the same channel, which limited the model to examine a maximum of 4 samples. However, due to its compact size, it could be adopted when limited environmental samples are to be examined for future studies. A better approach was to design a model which was capable of examining more environmental samples with minimal components, similar sample volume capacity and smaller sizes. The limitations from the current design were considered and improvements were incorporated in the following designs. 


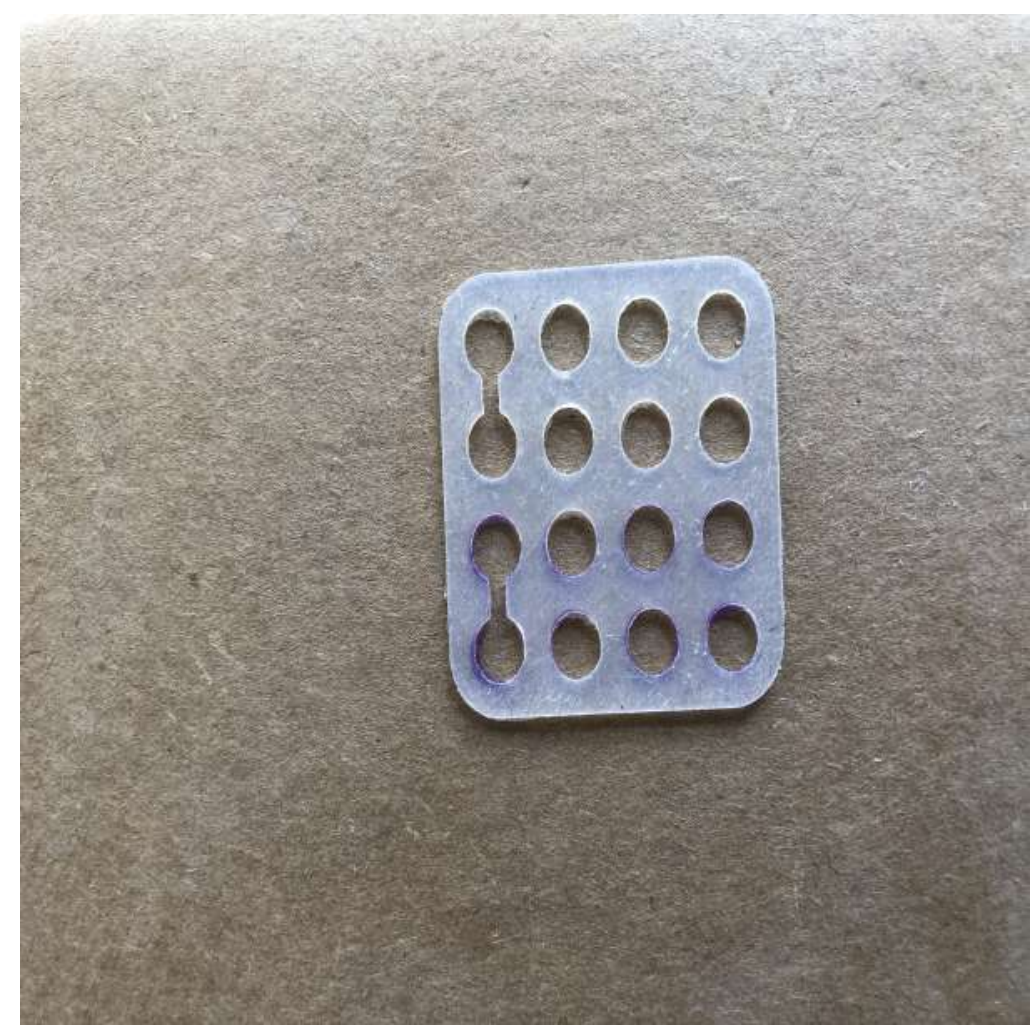

Figure 3.10: Silicone gasket for the first $\mu$-MAMA model; scale: $39 \mathrm{~mm} \times 39$ $\mathrm{mm} \times 0.5 \mathrm{~mm}$.

\subsubsection{Second Design}

The previous design was modified, taking into account the advantages of the first design, in order to meet the expectations. In this attempt, the geometry of the model was changed from square shaped to a hexadecagon shape model with each well placed in circular pattern as shown in figure (3.11) at an equal distance relative to each other. The model consisted of 16 wells with a sample volume capacity similar to that of the first model. From the figure, it can be seen that each well had its own inlet and outlet connections that made it possible to examine more environmental samples. By doing so, it made the design more robust, free of cross-contamination and leakage, reliable and easy to integrate into the instrument suite. Similar fittings and tubing were used for microfluidic connections, and same procedure was followed to seal the microassay plate. During the printing process, however, it was observed that the resin inside the channels was cured and therefore the channels were blocked as shown in figure (3.13). This could have happened 


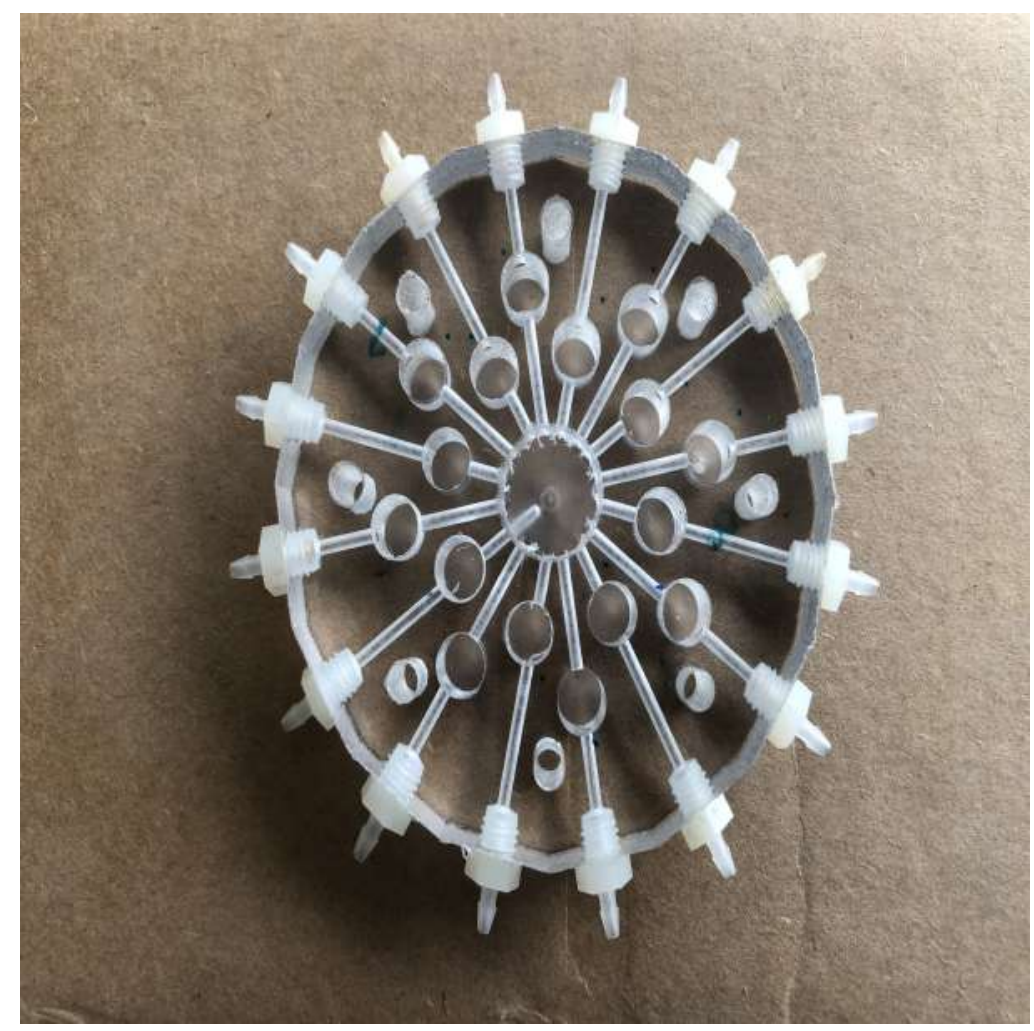

Figure 3.11: Base plate of the second $\mu$-MAMA model with tight seal barbed fittings; scale: $13 \mathrm{~mm}$ (height) $\times 68 \mathrm{~mm}$ (diameter).

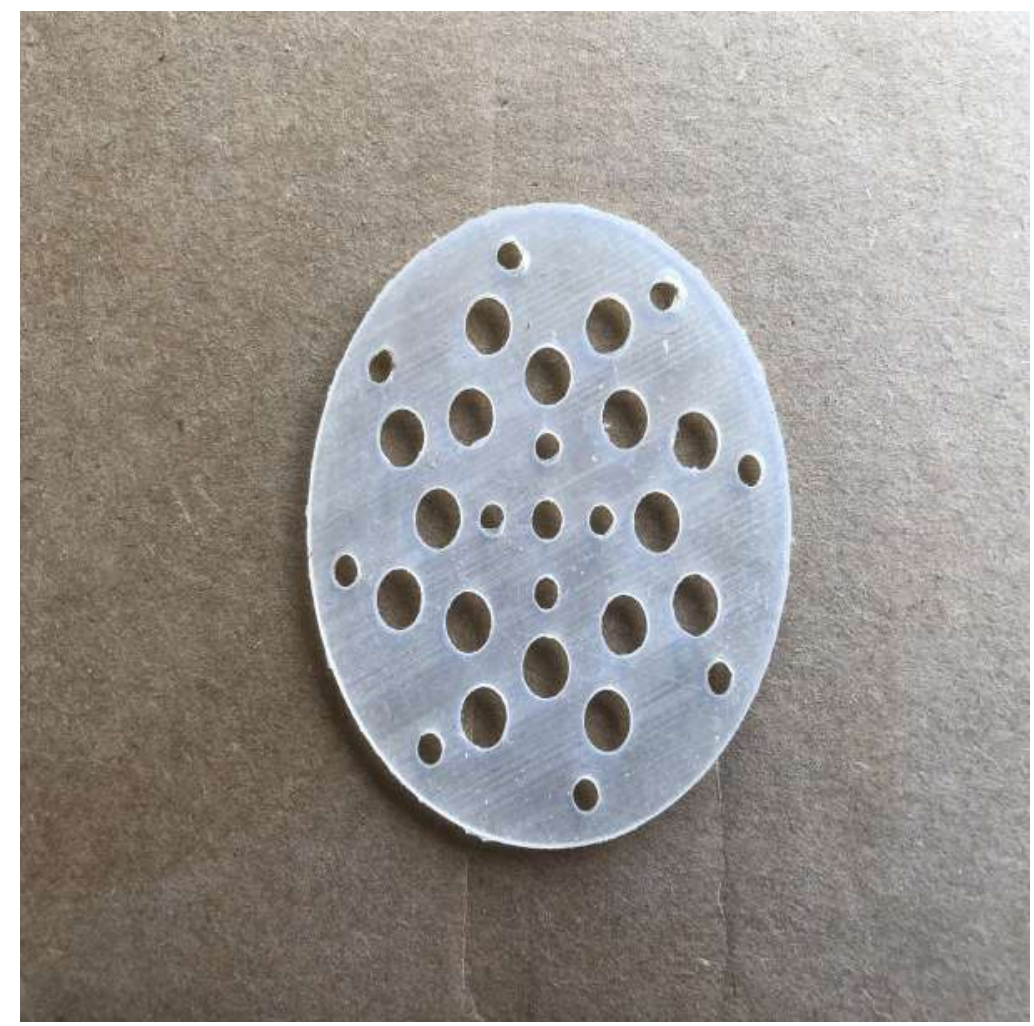

Figure 3.12: Assembly of the first $\mu$-MAMA model; scale: $1 \mathrm{~mm}$ (height) $\times 60$ $\mathrm{mm}$ (diameter). 
because of the nature of the SLA printer.

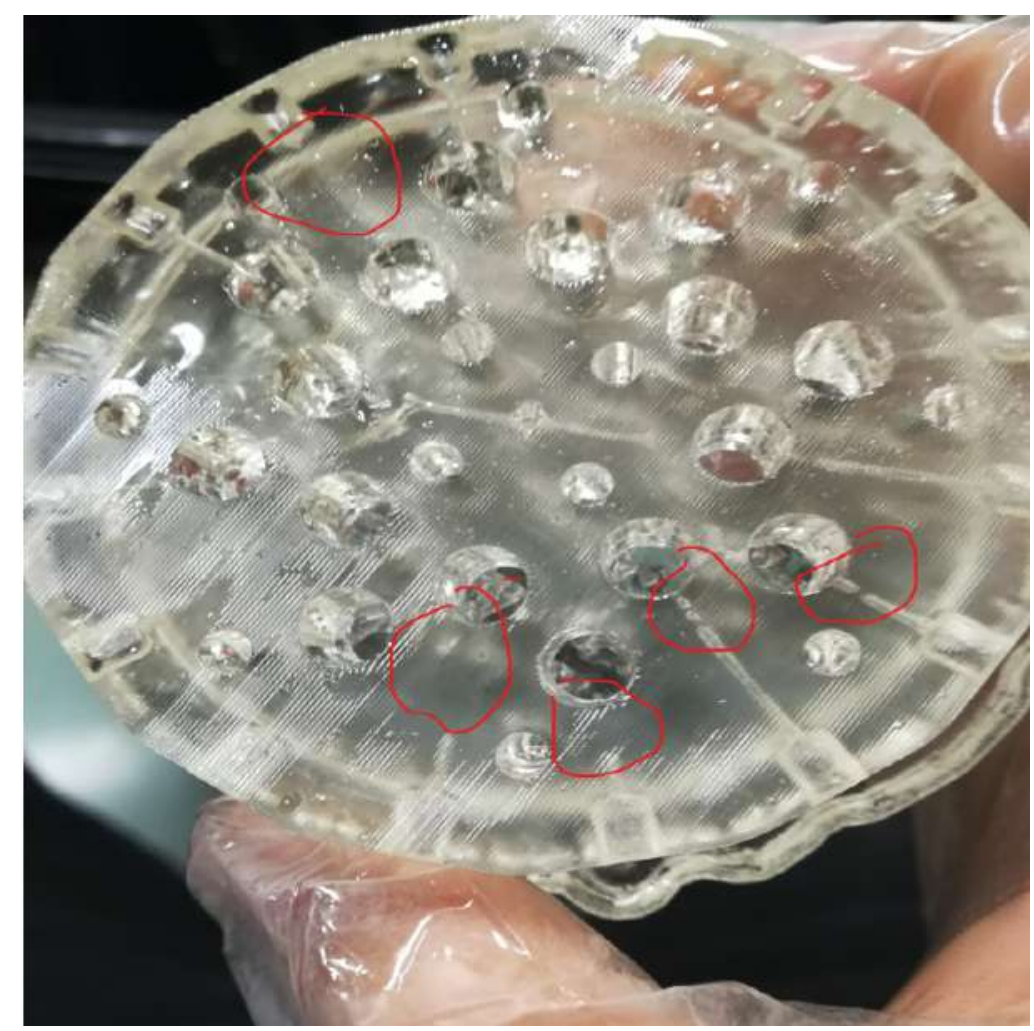

Figure 3.13: Channel blockage in the second design of $\mu$-MAMA model; scale: $13 \mathrm{~mm}$ (height) $\times 68 \mathrm{~mm}$ (diameter).

The resin accumulated inside the channel instead of flowing out of the channel. It was due to the resin viscosity that was entrapped by the capillary forces in the channel. As the current layer was printed, the resin inside the channels was cured by the UV light of subsequent layers due to the transparency of the cured resin because of which there was solid resin in the channel. The problem could be resolved if the channel were made perpendicular to the plane of gravity and this would help the resin flow out of the channel. Therefore, all the problems associated with this model were kept in mind and the following final model was designed.

\subsubsection{Final Design}

To cover up the limitation from the first two models, a final design as shown in figure (3.17) was printed with Veroclear transparent using a Polyjet printing technology. 


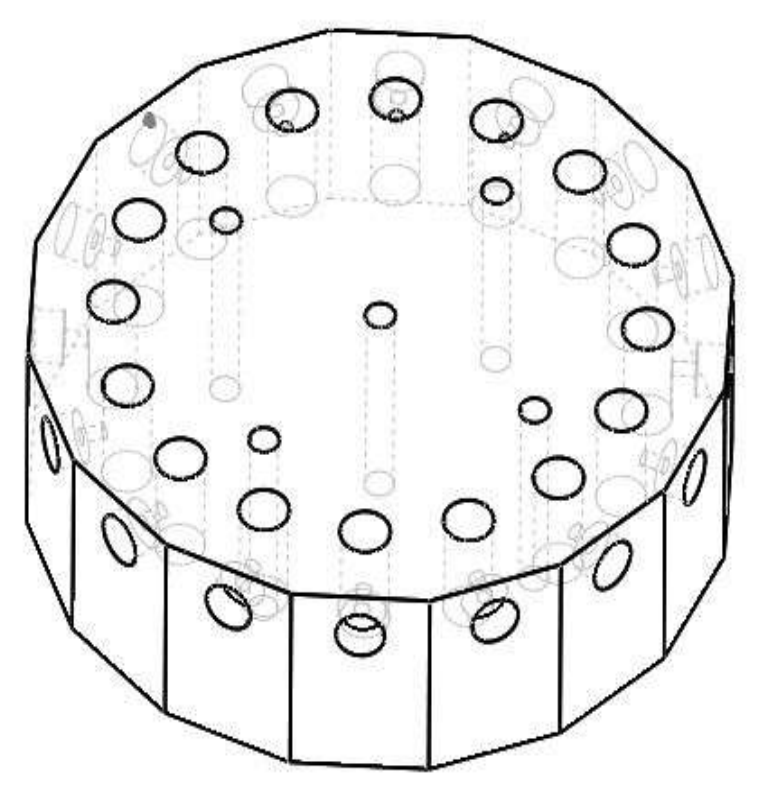

Figure 3.14: 3D design model of the final $\mu$-MAMA base plate; scale: $17 \mathrm{~mm}$ (height) $\times 68 \mathrm{~mm}$ (diameter).

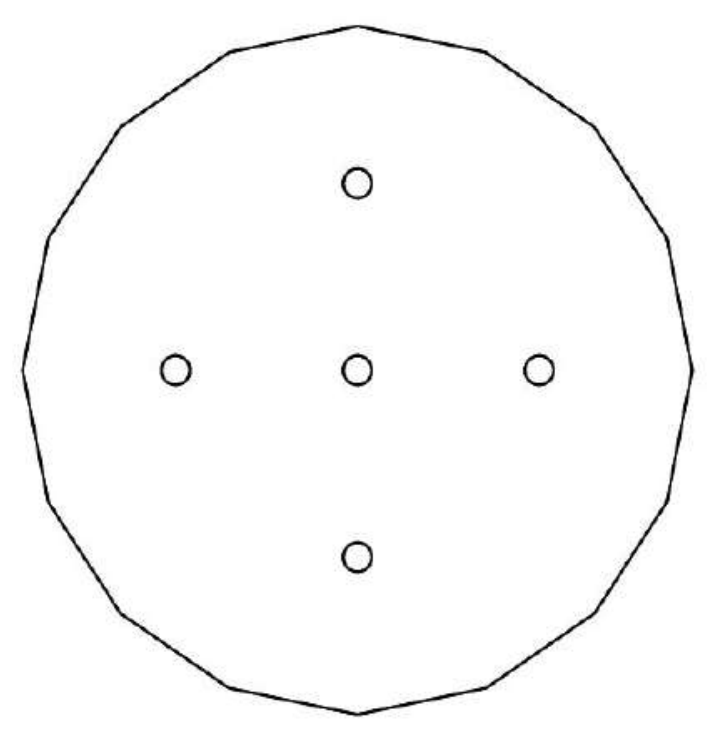

Figure 3.15: 3D design model of the final $\mu$-MAMA top plate; scale: $5 \mathrm{~mm}$ (height) $\times 68 \mathrm{~mm}$ (diameter) .

The idea of having each well with its own inlet and outlet significantly reduced the chances of cross-contamination. It was ensured that the channels were not 


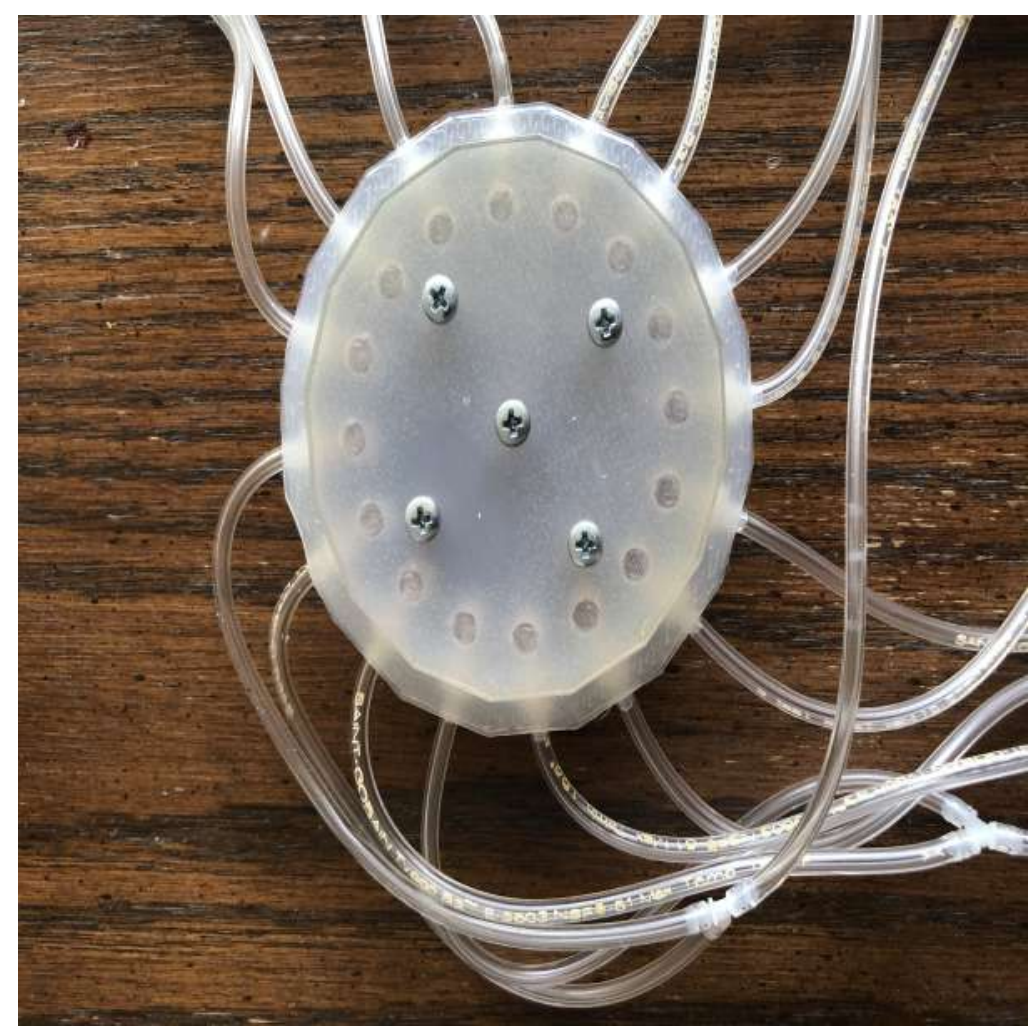

Figure 3.16: Assembly of the final $\mu$-MAMA design model; scale: scale: $24 \mathrm{~mm}$ (height) $\times 68 \mathrm{~mm}$ (diameter) .

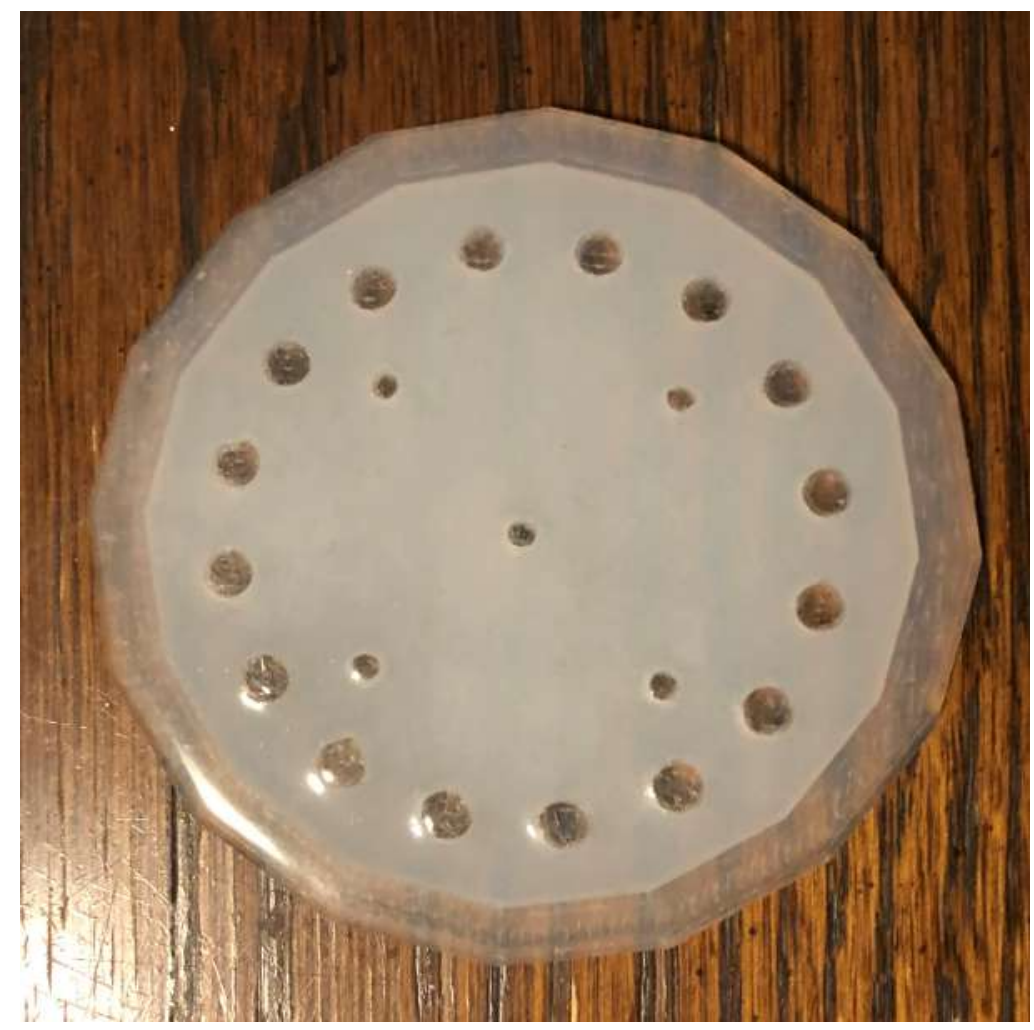

Figure 3.17: Silicone gasket for the final $\mu$-MAMA model; scale: $1 \mathrm{~mm}$ (height) $\times 68 \mathrm{~mm}$ (diameter). 


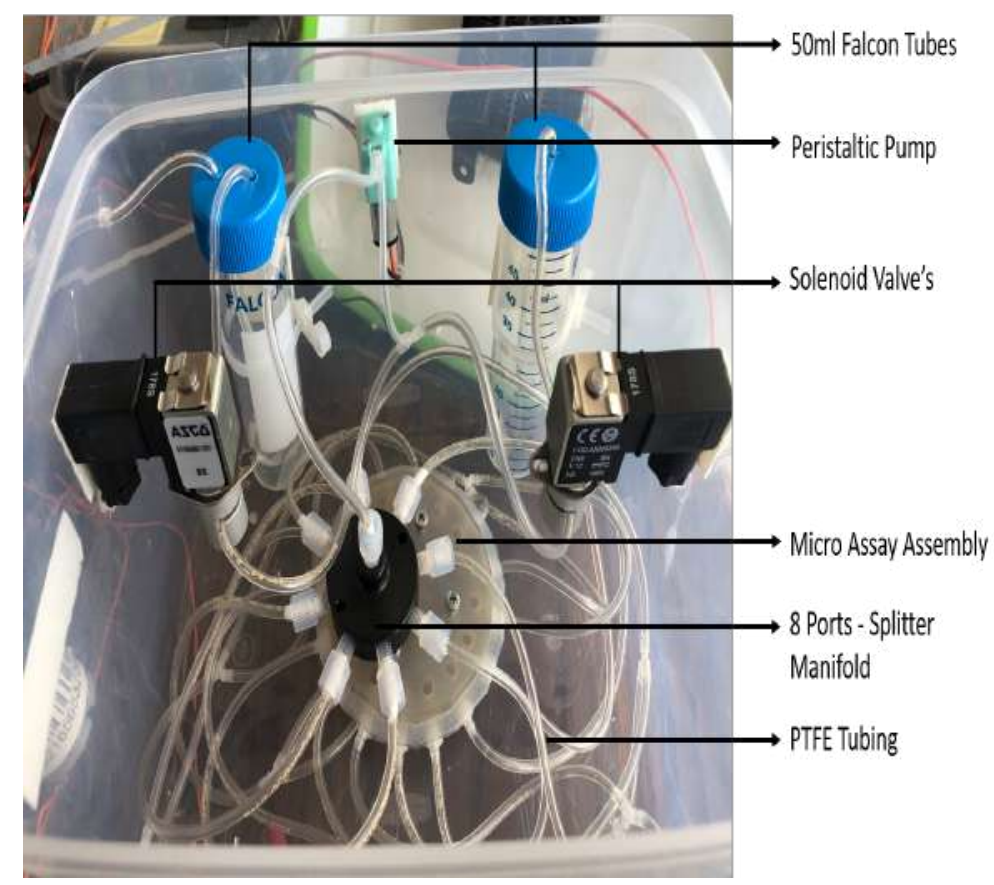

Figure 3.18: Zoomed-in view of the final $\mu$-MAMA sub-unit; scale: $200 \mathrm{~mm} \times$ $135 \mathrm{~mm} \times 165 \mathrm{~mm}$.

blocked as this could hinder the flow of fluid into the wells, resulting in a failure of the printed part. This design allowed to account for metabolic activity from a wide range of metabolisms, thus increasing the chances of detection. In addition, it was housed in a temperature-controlled chamber (see figure (3.18)) for in fieldincubation. This design was therefore finalised and incorporated for field testing.

\subsection{Nucleic Acid Extraction and Preparation}

Numerous automated and semi-automated methods are currently available for extraction of nucleic acid from low biomass environment (such as permafrost ice, subzero saline springs, sediments and endoliths) including the Claremont Biosolutions SimplePrep X1 [60], Promega Maxwell RSC [55] and milipore super FastPrep2 [104] for nanopore sequencing. From the experiments, it was observed that Claremont X1 and Promega RSC did not perform well as they left impurities and inhibitors in the extracted DNA making the downstream analysis such as sequencing and PCR difficult. Among these the best extraction method was the super FastPrep2, but only partial extraction (cell lysis) was possible and additional 
manual purification instruments were still required. In addition, these systems require relatively high volume, mass and power requirements which restrict their use outside the laboratory and ultimately as spaceborne instruments. Thus, there was a significant need for an ultra small, low energy automated DNA extraction instrument. The accepted method of amplifying and detecting DNA molecules is real-time polymerase chain reaction ( $\mathrm{qPCR}$ ), a sensitive technique suitable for microfluidic integration [105][106]. Its performance depends on previous DNA extraction from target cells, which in turn is dependent on cell wall disruption, especially when working with microorganisms that show resistivity to lyse such as Gram positive bacteria and fungi [107][108].

Generally, microfluidic integrated DNA extraction methods are bifurcated into two groups: chemical methods and physical methods. Chemical methods release cellular DNA by solubilizing membrane lipids and proteins using chaotropic agents such as guanidine thiocyanate [109][110] and biological enzymes [111] that are easy to implement as they do not require any additional equipment. However, a prior mixing step was required and this can be difficult to achieve on the chip as the flow regime is laminar. Furthermore, the chemicals can inhibit subsequent downstream reactions, thus requiring a thorough cleaning step [112]. In addition, physical methods require additional hardware components that increase the complexity of microsystem integration. Conversely they have many advantages, since they do not leave residual substances, they are faster and more efficient. Several physical lysis methods have been reported based on thermal treatment [113], sonolysis [114], electroporation [115], laser induced cell wall disruption [116] and mechanical lysis [117]. Among them, mechanical bead-based analysis was the commonly used method of working with hard-to-lyse samples due to its efficacy and reproducibility [118].

In an attempt to overcome above mentioned problems, a novel automated nucleic acid extraction system (A-NECS) was designed and manufactured on the basis of magnetic bead based extraction methodology. This method combined a 
magnetic stirrer and bead beating features to disrupt the cell wall of microorganisms that were hard to lyse. In order to minimize time consumption, the designed system worked in continuous flow performing lysis at flow rates ranging from 40 $\mu \mathrm{l} / \mathrm{min}$ to $200 \mu \mathrm{l} / \mathrm{min}$. Its ability to process large volumes of fluid prevented the use of both chemical reagents and heat that can inhibit downstream PCR reactions and/or protein analysis.

\subsubsection{Device Fabrication}

Automated nucleic acid extraction system (A-NECS) chips were manufactured using 3D polyjet printing technology for Veroclear transparent material. Polyjet printing was chosen because of its ability to produce smooth and accurate parts. It was capable of producing thin channels and complex geometries in the chip with a microscopic layer resolution of $0.014 \mathrm{~mm}$. Two chips were manufactured, one with baffle regions and the other without baffle regions as shown in figures (3.20) and (3.24). Zirconia/silica beads from Biospec and Neodymium block magnets (N35) with dimensions $19.05 \times 6.35 \times 8.37 \mathrm{~mm}$ with radial magnetisation were housed in a lysis chamber of $\sim 2385 \mu \mathrm{l}$ and sealed with pressure sensitive adhesive, silicone sealing gaskets (see section 3.4.3) and top plate. The lyse chamber connected the sample inlet and outlet via $1600 \mu \mathrm{m}$ channels. The channels were made smaller to reduce the fluidic resistance and at the same time worked as a bead weir, as the smallest beads were larger than $1600 \mu \mathrm{m}$ in diameter. 10-32 UNF threading was made for the fluidic connections to the sample inlet and outlet.

\subsubsection{First Microfluidic Chip with Baffle}

The first chip design consisted of two reservoirs, a lysing chamber, baffle, 1032 UNF thread microfluidic connections and four through holes at the corners as depicted in the figure (3.22). Holes were drilled to help bond the top plate, silicone gasket and bottom plate together with nut and bolt. The two plates, together with the silicone, were pressed together to form a permanent bond. The micro- 


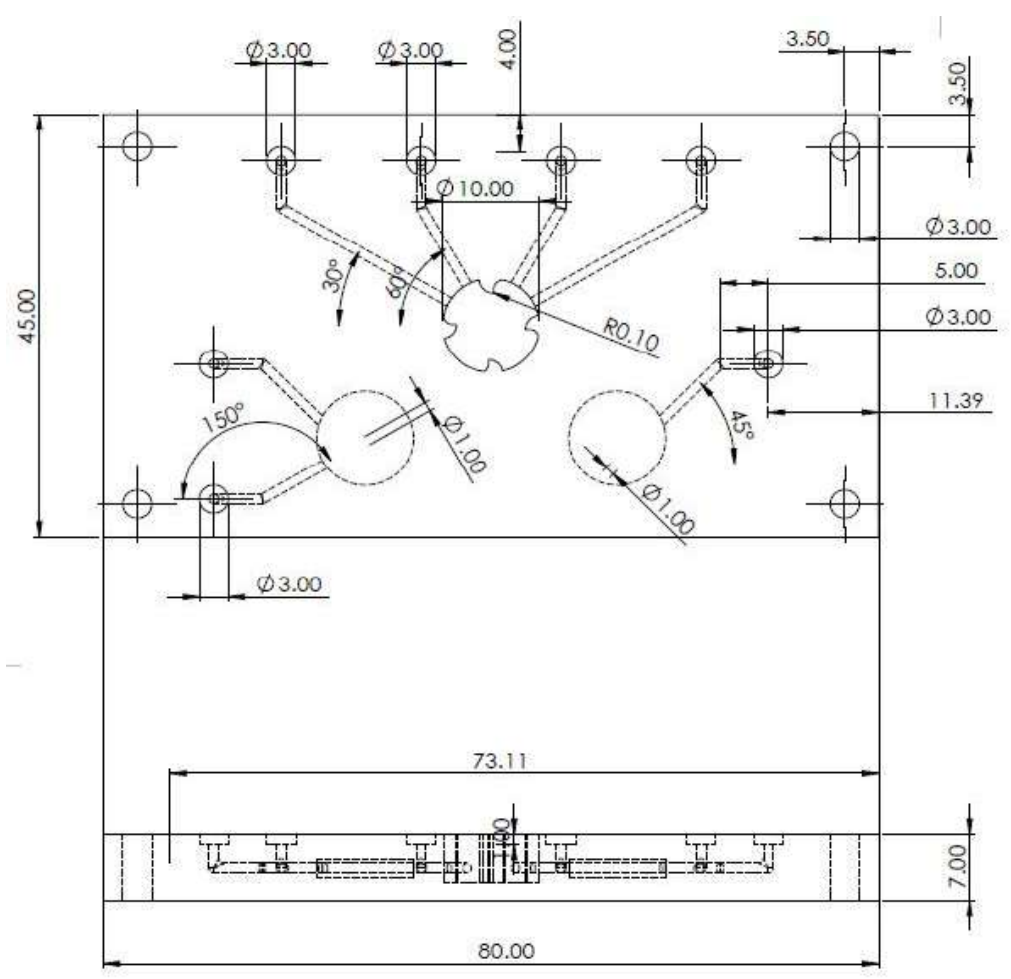

Figure 3.19: Top and side views of the first microfluidic chip with baffle dimensions; all dimensions are in $\mathrm{mm}$.

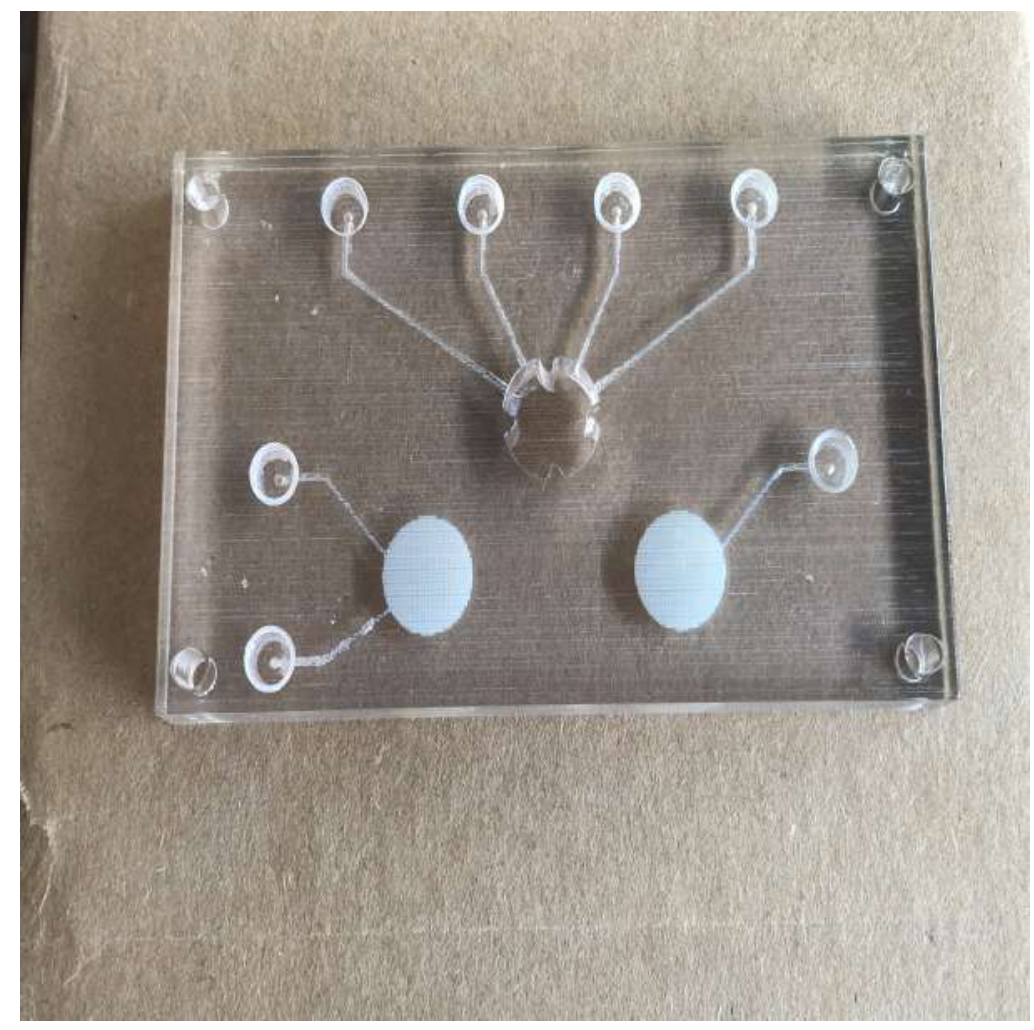

Figure 3.20: 3D printed bottom part of the $1^{\text {st }}$ microfluidic chip with baffle; all dimensions are in $\mathrm{mm}$. 


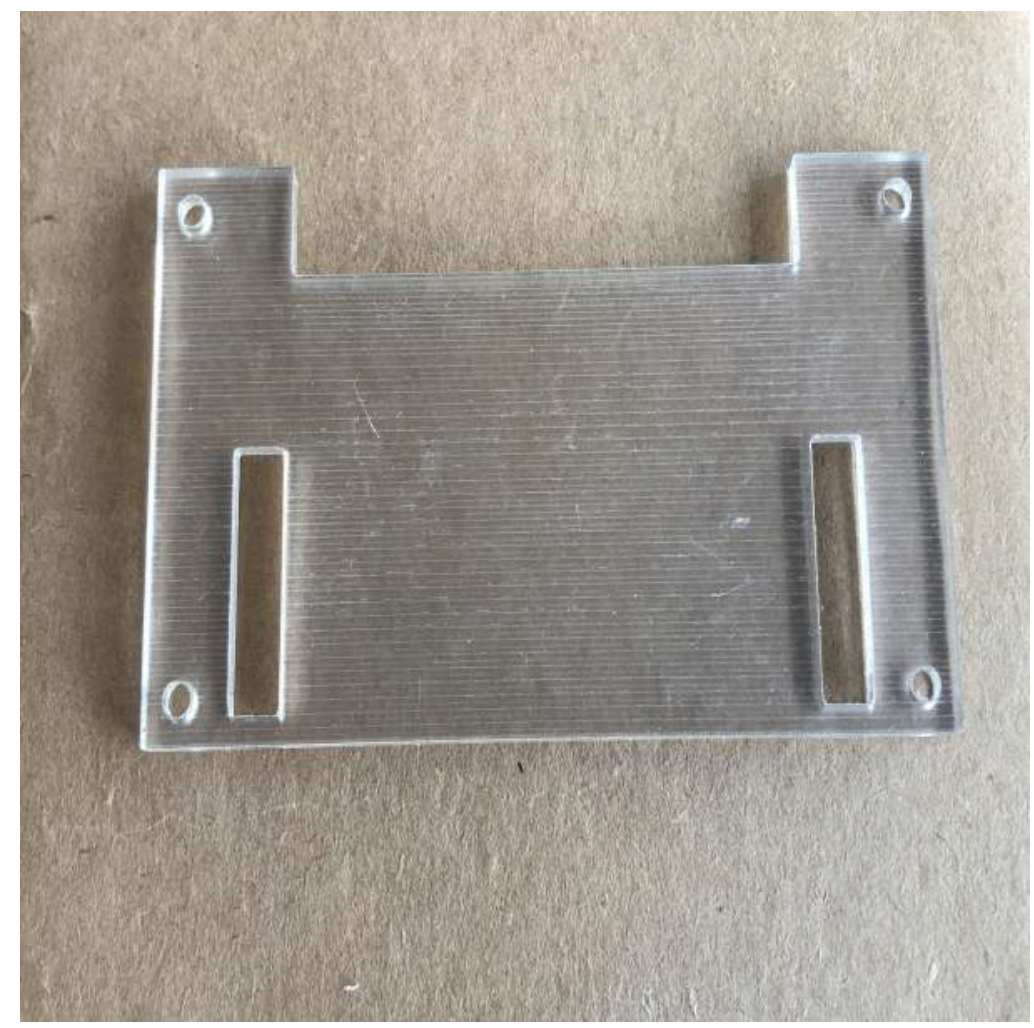

Figure 3.21: 3D printed top part of the $1^{\text {st }}$ microfluidic chip with baffle; scale: $80 \mathrm{~mm} \times 45 \mathrm{~mm} \times 3 \mathrm{~mm}$.

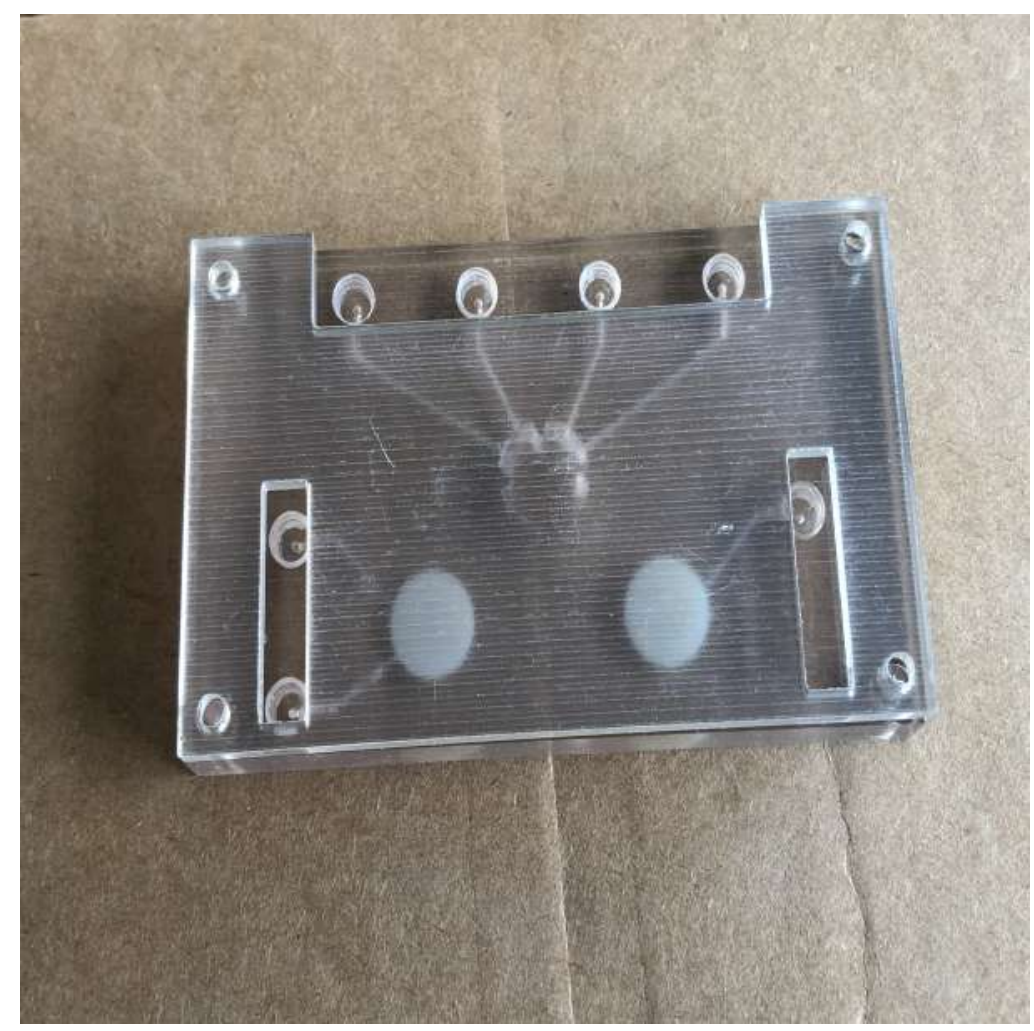

Figure 3.22: Assembly of the $1^{\text {st }}$ microfluidic chip with baffle; scale: $80 \mathrm{~mm} \times 45$ $\mathrm{mm} \times 10 \mathrm{~mm}$. 
fabrication process produced four $1 \mathrm{~mm}$ diameter channels that were connected to the lysing chamber. The left reservoir, which was an additional reservoir, was designed with a diameter of $10 \mathrm{~mm}$ and a depth of $2 \mathrm{~mm}$ to hold larger volumes of fluid $(\leq 1 \mathrm{ml})$ when needed. On the other hand, the right reservoir was used to hold the sample or reagent temporarily when it was transferred from one of the tubes connected to the system to the lysing chamber. Finally, the purpose of introducing a baffle in the lysing chamber was to enhance the mixing process and to help extract the DNA from the sample by disrupting the cell wall. However, after experimenting with the current design model, it was noted that the introduction of a baffle did not have a significant effect on the mixing process and instead reduced efficiency because it hindered the rotation of the magnetic stirrer, which resulted in a reduced magnetic rotational speed.

\subsubsection{Final Microfluidic Chip without Baffle}
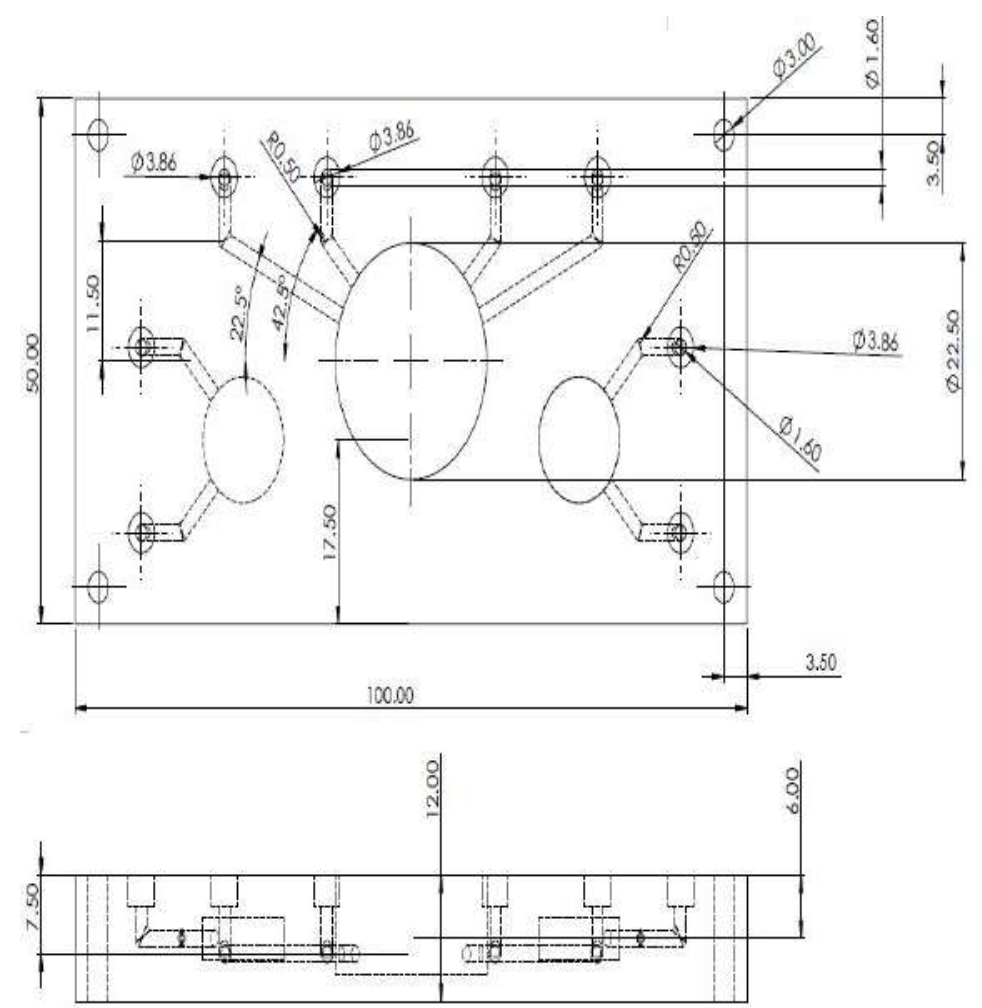

Figure 3.23: Top and side views of the final microfluidic chip with baffle dimensions; all dimension are in $\mathrm{mm}$. 
The observations made on the first chip were considered and the necessary modifications to the design of the first chip were made in order to produce better results. In this design, the baffles which did not have a significant effect on the mixing were removed and a larger magnet of $19.05 \times 6.35 \times 8.37 \mathrm{~mm}$ with radial magnetisation was accommodated in the lysing chamber. In order to accommodate a larger magnet, the size of the chamber was increased by $22.5 \mathrm{~mm}$ in diameter and $9.5 \mathrm{~mm}$ in height. A more detailed dimensional model is shown in the figure (3.23). In addition, an additional inlet/outlet for the reservoir on the right of the lysing chamber was introduced to prevent all cases of cross-contamination and the formation of bubbles.

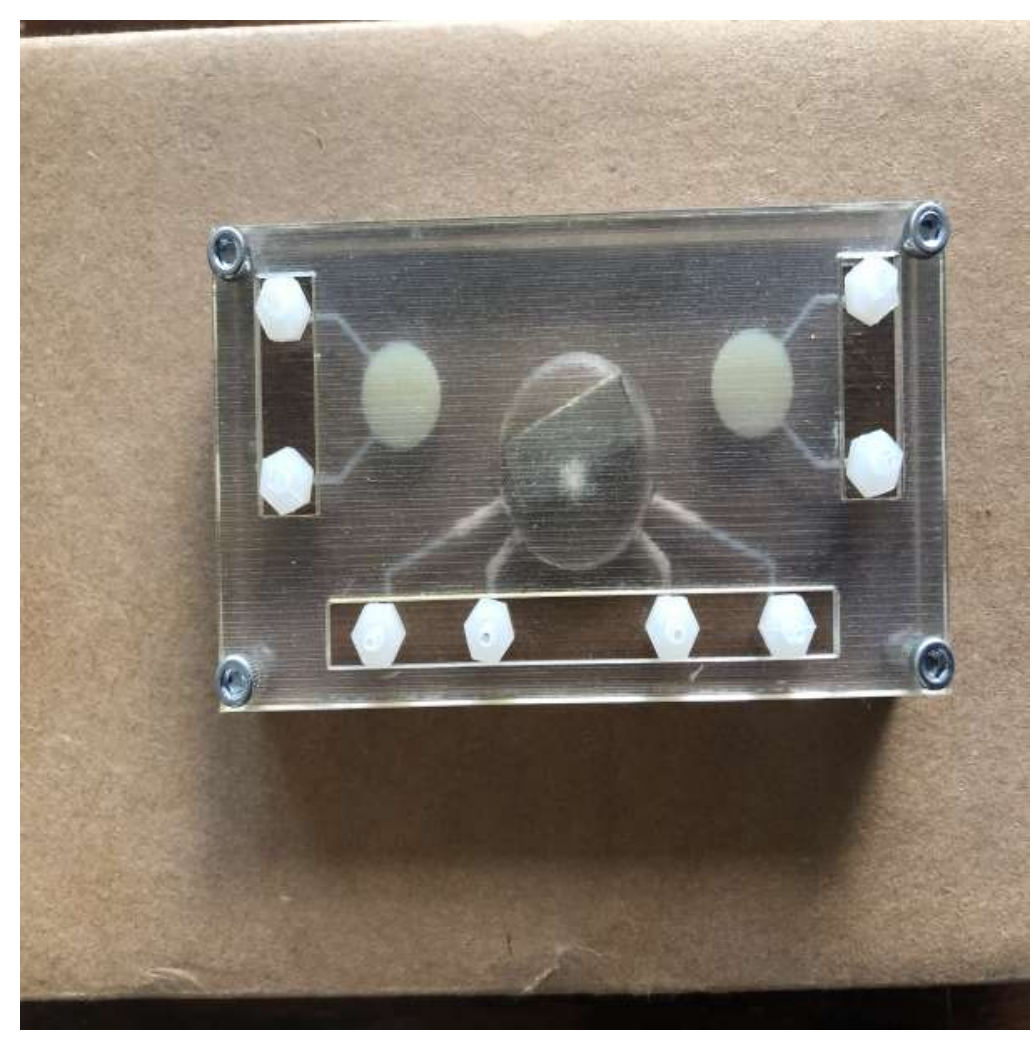

Figure 3.24: Assembly of the second microfluidic chip without baffle; scale: 100 $\mathrm{mm} \times 50 \mathrm{~mm} \times 15 \mathrm{~mm}$.

\subsection{Summary}

The methodology used to develop an automated microfluidic device was described in this chapter, which contributes to the first and third objectives of this thesis. 
A detailed explanation for the proof-of-concept prototype was provided for the different stages of the design and its deployment. Before moving to the next design model, the operating limits of each designed microassay were observed and kept in mind, and the material best suited for microfluidic applications was discussed. It was concluded that both, the $\mu$-MAMA and the A-NECS models used were capable of predicting microbial activity and nucleic acid extraction from cryoenvironment samples. Finally, A-NECS chip fabrication was discussed that helped develop an understanding about the sample size that can be treated and the ideal rotational speed of the magnet stirrer.

Computational fluid modelling of the lysing chamber is described in the next chapter, followed by the model simulation results. 


\section{Chapter 4}

\section{CFD Modeling and Simulations}

The $\mu$-MAMA and A-NECS design processes were discussed in the previous chapter and a mathematical model associated with the microfluidic flow was established. This chapter focuses on the computational dynamics of fluid flow modelling in a lysing chamber. A brief description of the problem and the type of method used is discussed in the section 4.1. The importance of two-compartment representation and the turbulent kinetic energy dissipation rate, $\varepsilon$ follows. Section 4.3 details the geometry followed by the grid sensitivity analysis in the section 4.4. The control equations used for the calculation of turbulent kinetic energy and kinetic energy dissipation rates are discussed in section 4.5. Section 4.6 documents the advantages of using the Multiple Reference Method (MRF) over the other methods available. Finally, section 4.7 discusses the case study used to validate and compare the results of the current simulation.

A number of CFD simulations of turbulent flow in the stirred chamber were performed to study flow behaviour, circular patterns, vortic structures, etc and this analysis helped to add theoretical depth to the design of the instrument. Among the various turbulent models studied, the transport turbulence model of average Navier-Stokes equations was the basis for most CFD simulations. Two turbulence models, namely $k-\varepsilon$ realisable and $k-\omega$ SST, are examined at different angular velocities in order to understand the turbulent properties, the volume density 
distribution of the dissipated energy and the effect of the agitation rate.

\subsection{Problem Description}

In all previous works, CFD simulations consisted of a propeller instead of a magnetic stirrer, making it an interesting area to explore. The distribution of the particle size of the droplets is one of the most important parameters for the liquidliquid suspension system. Population balance was used to predict the size of the particle distribution of droplets in different zones. Some parameters of the population balance model, such as the turbulent dissipation rate, were derived from the CFD simulations of the turbulent flow field. The lysing chamber was divided into two zones, namely a magnetic zone with a high local energy dissipation rate, and a relatively large circulation zone with a low energy dissipation rate with specific turbulent properties. Population balance equations have been resolved for each zone and PSD for the entire chamber was obtained. The objective of the two zone model was to provide turbulence parameters for the different zones to be used by the population balance model for accurate PSD prediction. To do so, the multiple reference frame (MRF) method was used for simulation purposes.

\subsection{Two Compartment Representation}

The compartment model has proved to be a successful tool in the biochemical simulation study. Compartment models of non-ideal mixing flows in agitated chambers are based on the non-homogeneous characteristics of turbulent flow. Although different parameters of turbulent flow could be used, the most characteristic feature of turbulent flow, which determines many mixing processes, is the turbulent kinetic energy dissipation rate, $\varepsilon$. The rate of energy dissipation affects micro-mixing and the rate of heat and mass transfer, which is crucial in the mixing chambers. Large localised energy dissipation rates could cause cellular damage and reduce chamber output. 
Considering the discrepancies in the existing experimental data on the amount of energy dissipated in each zone, the simplest possible representation of a nonhomogeneous turbulent stirred chamber is to consider only two homogeneous compartment: a small region around the magnet and a large circulation zone far from the magnet zone.

\subsubsection{Determination of Two Compartment Parameters}

The two compartment parameters discussed by Alexopoulos et al. [119] were used to compare the validation results of this study. In order to determine these parameters, the results of the CFD simulations were post-processed to extract the volume density distribution of the energy dissipation rate within the lysing chamber, which provided a concise representation of the non-homogeneity of $\varepsilon$. The volume density distribution over a discrete range of dissipation rates was obtained by summing up all the grid cells that had the energy dissipation rate within the chosen section, taking into account the respective grid-cell volume:

$$
n_{i}=\sum_{j=1}^{N_{t o t}} \frac{V_{j}\left(H\left(\varepsilon_{j}-\varepsilon_{i-1}\right)-H\left(\varepsilon_{j}-\varepsilon_{i}\right)\right)}{V_{t o t}}
$$

where $V_{j}$ is the volume of the $j^{t h}$ cell, $N_{\text {tot }}$ is the total number of grid cells, and $H(x)$ is the Heaviside function. Since the number of sections that represented the energy dissipation rate was smaller than the total number of grid cells, the volume density distribution $n_{i}$ was much more efficient than simple energy dissipation rate values.

Another effective way to predict zone boundaries was through the use of cumulative energy dissipation rates. Cumulative energy dissipation is the total energy dissipated between $\varepsilon_{\min }$ and $\varepsilon_{i}$ which can be defined [119] over the discrete range of dissipation rates:

$$
\left.E_{i}=\sum\right]_{j=1}^{i} \varepsilon_{j} n_{j}
$$


where $\varepsilon_{j}$ is the energy dissipation rate and $n_{j}$ is the volume density distribution corresponding to that section.

\subsection{Geometry of the Model}

CFD simulations were conducted in a $9.5 \mathrm{~mm}$ high, $22.5 \mathrm{~mm}$ diameter cylindrical chamber filled with water and dye reagent at a ratio of 9:1 up to a height of 9 mm, equipped with a magnetic stirrer of $19.05 \mathrm{~mm}$ in length $\times 6.35 \mathrm{~mm}$ in width $\times 8.37 \mathrm{~mm}$ in height as shown in figure (4.1).
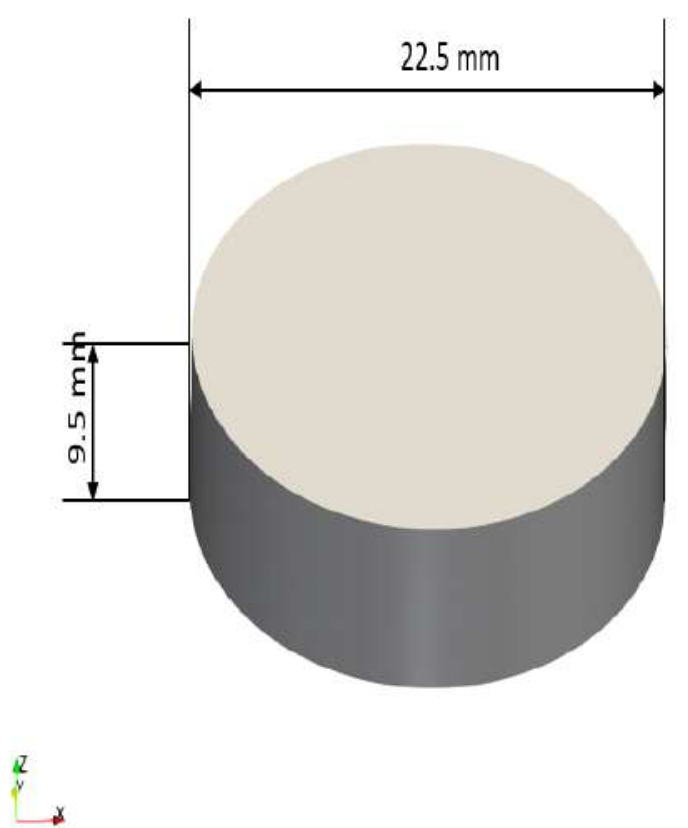

Figure 4.1: Model geometry for CFD simulations.

\subsection{Grid Generation}

Due to the Multiple Reference Method (MRF) for simulation, the volume of the chamber was divided into two cylindrical zones. The inner zone comprised a magnetic stirrer and the outer zone included the remaining chamber. An unstructured grid with tetrahedral elements consisting of approximately 968,596 elements and 
an element size of $1 e^{-5} \mathrm{~mm}$ was used in this study as shown in figure (4.2). The grid was refined near the magnetic zone to capture the turbulent properties. Three grids with different element sizes as shown in the table (4.1) were used to establish mesh independence. From the figure (4.3) it can be seen that there were negligible differences between the predictions of the last two mesh sizes.

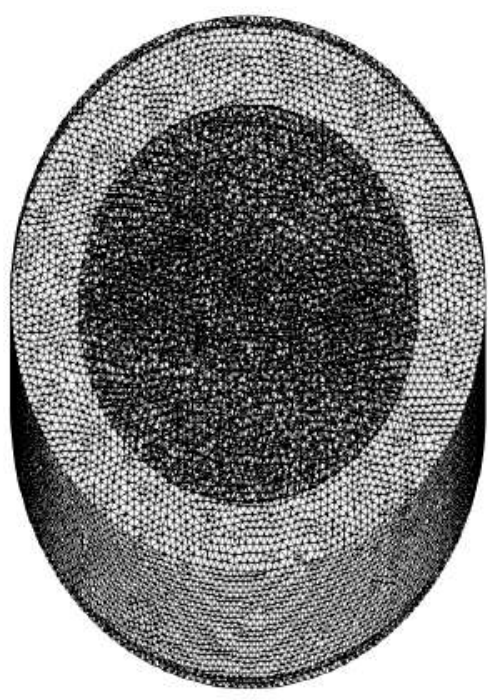

Figure 4.2: Computational domain discretization into a unstructured tetrahedral mesh.

Table 4.1: Grid independent study for different element sizes.

\begin{tabular}{|c|c|c|}
\hline Type of Mesh & $\begin{array}{c}\text { Element size } \\
(\mathbf{m m})\end{array}$ & $\begin{array}{c}\text { Y-velocity } \\
\text { at } \mathbf{x}=\mathbf{9 . 7} \mathbf{~ m m}\end{array}$ \\
\hline Coarse & $1 e^{-3}$ & $1.22045 \mathrm{~m} / \mathrm{sec}$ \\
\hline Medium & $1 e^{-4}$ & $1 \mathrm{~m} / \mathrm{sec}$ \\
\hline Fine & $1 e^{-5}$ & $1 \mathrm{~m} / \mathrm{sec}$ \\
\hline
\end{tabular}




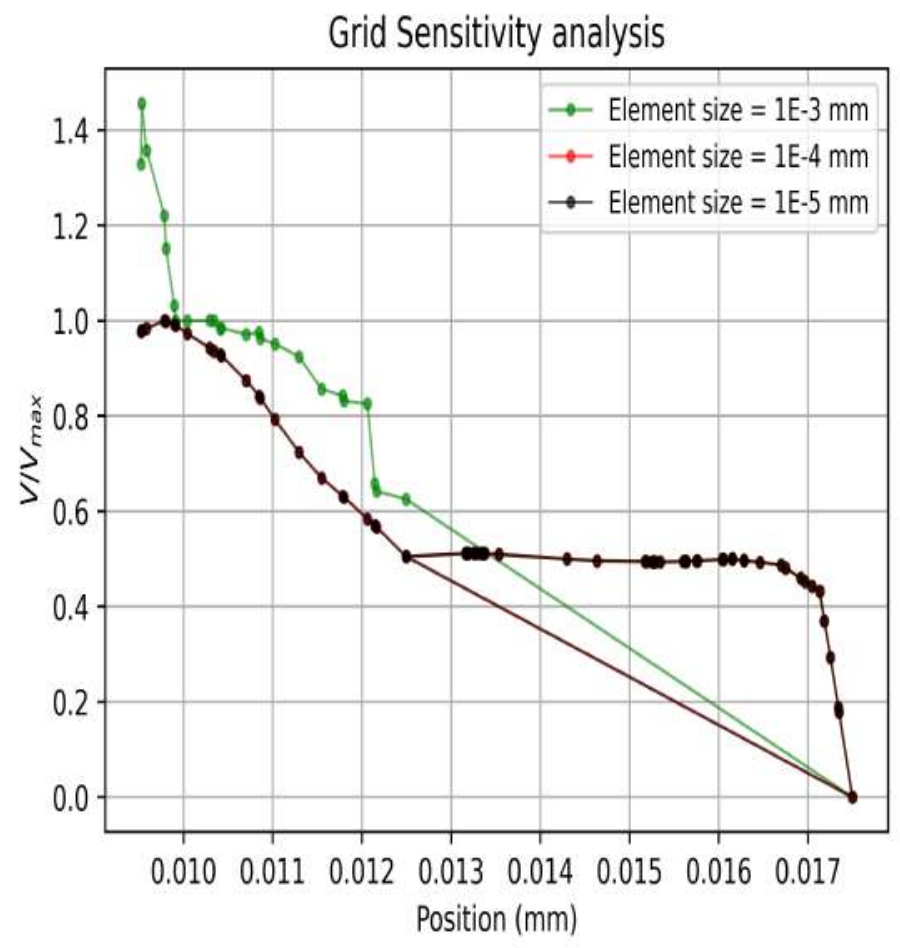

Figure 4.3: Y-velocity distribution along the magnet zone $\mathrm{x}$ axis.

\subsection{Governing Equations}

Governing equation for the incompressible fluid flow are:

$$
\frac{\partial \bar{u}_{j}}{\partial x_{j}}=0
$$

The above equation is know as the continuity equation. The momentum equation for this case is:

$$
-\frac{\partial}{\partial x_{j}}\left(\rho \overline{u_{j}} \overline{u_{i}}\right)-\frac{\partial}{\partial x_{j}}\left(\rho \overline{u_{j}^{\prime} u_{i}^{\prime}}\right)+\mu \nabla^{2} \overline{u_{i}}-\frac{\partial \bar{P}}{\partial x_{i}}+\rho g_{i}=0
$$

In the equation (4.4) $\rho \overline{u_{j}^{\prime} u_{i}^{\prime}}$ is the stress of Reynolds that can be modelled by semi-empirical relationships. In this work, two equations $k-\varepsilon$ model and $k-\omega$ SST model were used. The governing equations for turbulent kinetic energy and 
kinetic energy dissipation [120] are as follows:

$$
\begin{gathered}
\rho \bar{u}_{j} \frac{\partial k}{\partial x_{j}}=\tau_{i j}^{(t)} \frac{\partial \bar{u}_{i}}{\partial x_{j}}-\rho \varepsilon+\frac{\partial}{\partial x_{j}}\left[\left(\mu+\frac{\mu^{(t)}}{\sigma_{k}}\right) \frac{k}{\partial x_{j}}\right] \\
\rho \overline{u_{j}} \frac{\partial \varepsilon}{\partial x_{j}}=C_{E 1} \frac{\varepsilon}{k} \tau_{i j}^{(t)} \frac{\partial \bar{u}_{i}}{\partial x_{j}}-C_{E 2} \rho \frac{\varepsilon^{2}}{k}+\frac{\partial}{\partial x_{j}}\left[\left(\mu+\frac{\mu^{(t)}}{\sigma_{\varepsilon}}\right) \frac{\partial \varepsilon}{\partial x_{j}}\right]
\end{gathered}
$$

where $C_{E 1}=1.44 ; C_{E 2}=1.92 ; C_{\mu}=0.09 ; \sigma_{k}=1$ and $\sigma_{\varepsilon}=1.3$

A no-slip boundary condition was used for liquid in contact with a solid surface. Zero shear stress was used on the free surface. The velocity gradients were set to be the same at the interface between the inner and outer zones.

\subsection{MRF Method for CFD Simulations}

Modeling problems involving both stationary (e.g. external lysing chamber) and moving (e.g. magnet) zones may be addressed in three ways, the Multiple Reference Method (MRF), the Mixing Plane Method and the Sliding Mesh Method. Among those, the MRF method was the simplest, and this approach is appropriate when the flow at the boundary between the inner and outer zones is almost uniform. Because the baffle was not considered during simulations, the use of the MRF method was an ideal choice.

Ansys CFX [121] software was used to perform calculations. The first order upwind discretion scheme was used for the convection term of momentum, turbulent kinetic energy, and energy dissipation rate equations. Relative velocity formulation and two different turbulence models were used to simulate a chamber containing a mix of water and dye. To use the MRF approach, a rotating coordinate system was adopted for the inner zone called fluid 1; the rotating rate was set equal to the magnet agitation rate, and a non-moving coordinate frame was defined for outer zone, including baffles called fluid 2. Angular velocity of magnet was set to zero in relation to the rotating coordinate system. Initial guesses for 
velocity, pressure and turbulence parameters were specified to start calculations. Water density values, $\rho_{w}$ and dye density values, $\rho_{d}$ were taken as 998.2 and 1250 $\mathrm{kg} / \mathrm{m}^{3}$. Dynamic water viscosity, $\mu_{w}$ and dye, $\mu_{d}$ were used as $0.001003 \mathrm{~kg} / \mathrm{m}-\mathrm{s}$. At the first step of the calculation, the angular velocity of the rotating reference frame was set as $15 \%$ of the case under investigation. After 2000 time steps, the results were saved and used as an initial estimate. This procedure helped to avoid any divergence in the solution. Convergence was achieved when residuals on continuity, velocity, kinetic energy and energy dissipation rates were all lower than $10^{-6}$.

\subsection{Model Validation}

To validate the model calculations, results were compared with the work done by Alexopoulos et al. [119]. The domain used in his work is significantly larger than the domain used in this research. Figures (4.4), (4.5), (4.6), and (4.7), show the velocity vectors and contours of turbulent kinetic energy dissipation rate obtained from Alexopoulos et al [119]. Figures (4.8), (4.9), (4.10) and (4.11) show the contours of velocity, total pressure, turbulent kinetic energy contours and velocity vector plot obtained from the $k-\varepsilon$ realisable model used in current research.

The results of this research were comparable to the work of Alexopoulos et al. [119]. The density of the turbulent kinetic energy dissipation rate of the contours around the magnetic tip was observed to be very large relative to the other points. This showed that the gradient of the energy dissipation rate near the magnetic tip was large. In addition, the magnitude of the velocity around the magnet tip was much higher than the rest of the fluid.

Shear Stress Transport (SST) turbulence model, which is a two equation eddyviscosity model, was chosen because it switches between $k-\omega$ and $k-\varepsilon$ based on the flow behaviour [122]. The wall boundary state of the $\mathrm{k}$ equation in the $k-\omega$ models is viewed in the same way as when improved wall treatments are used in 


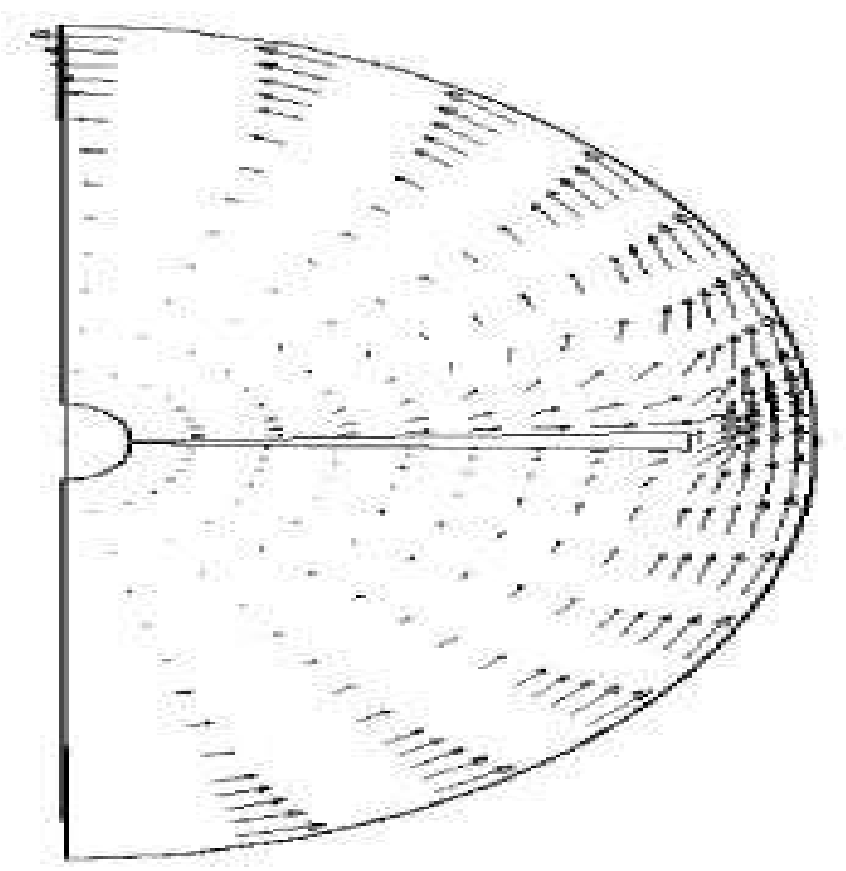

Figure 4.4: Velocity vectors plot in the XZ plane from Alexopoulos et al. [119].

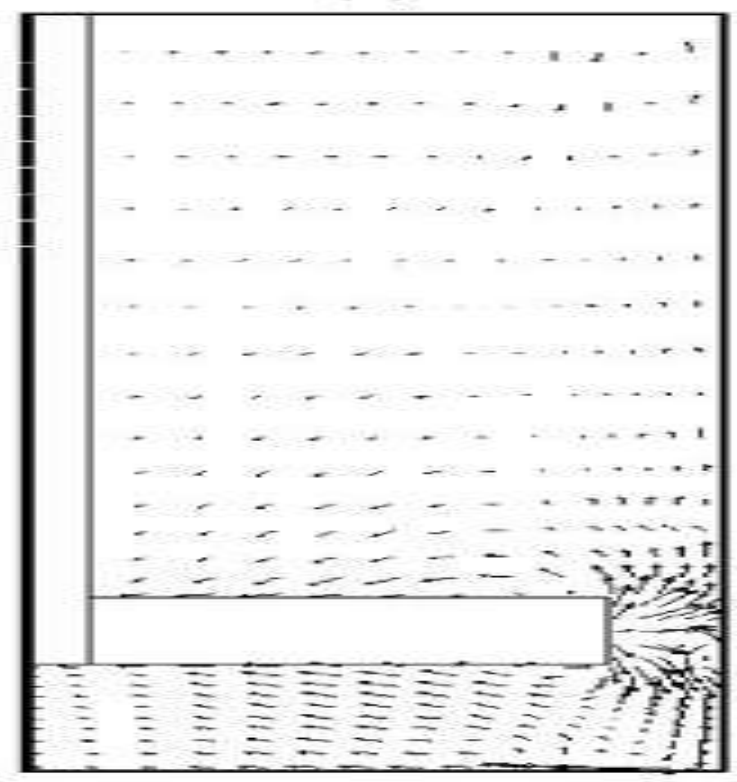

Figure 4.5: Velocity vectors plot in the XY plane from Alexopoulos et al. [119]. 


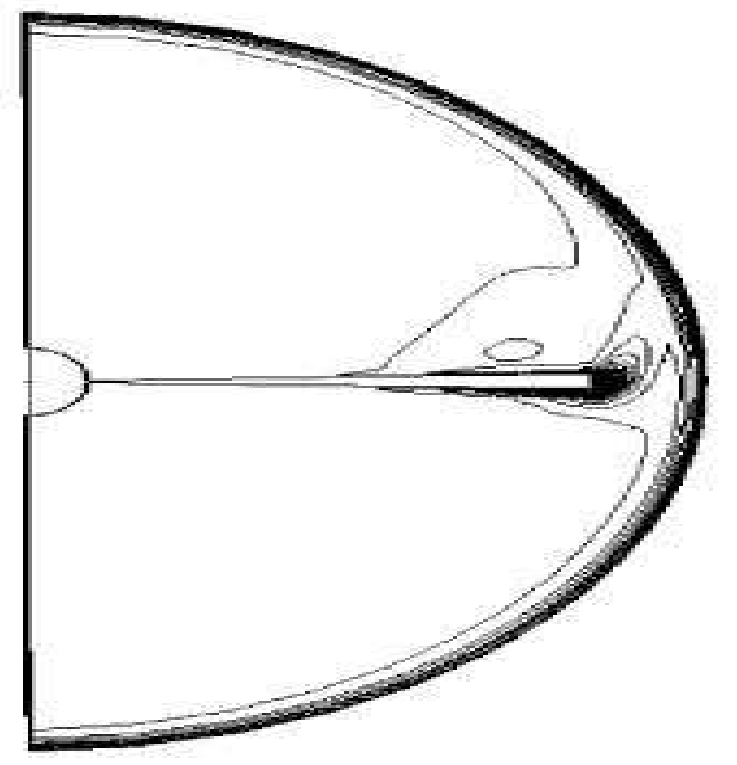

Figure 4.6: Energy dissipation rate contour in the XZ plane from Alexopoulos et al. [119].

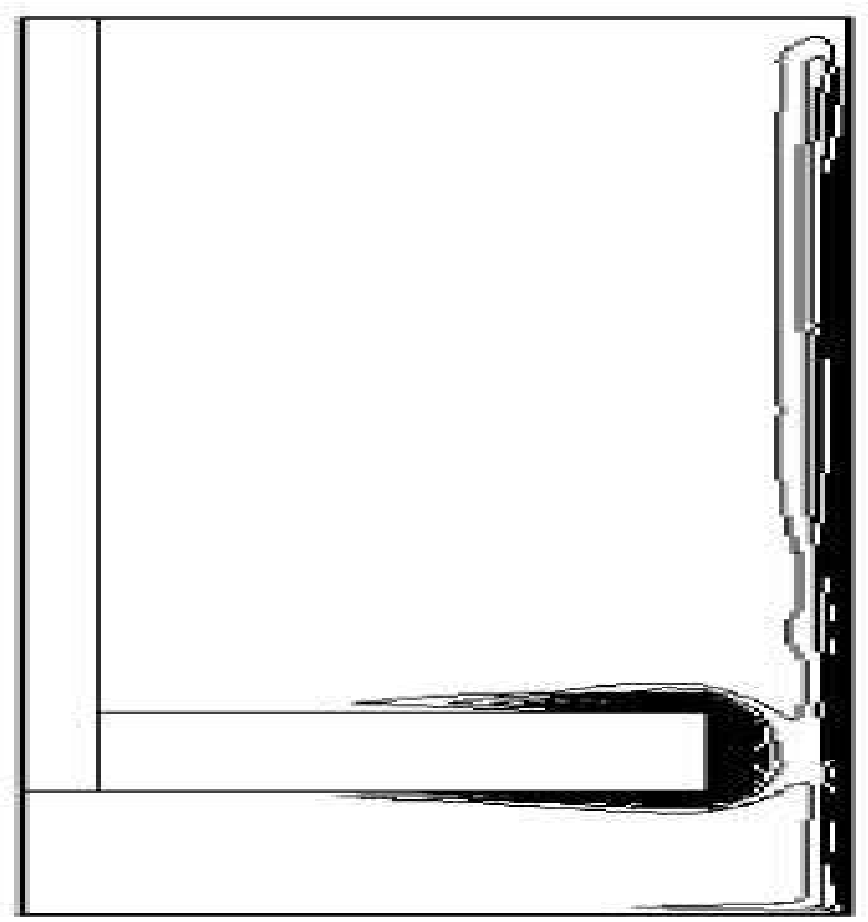

Figure 4.7: Energy dissipation rate contour in the XY plane from Alexopoulos et al. [119]. 


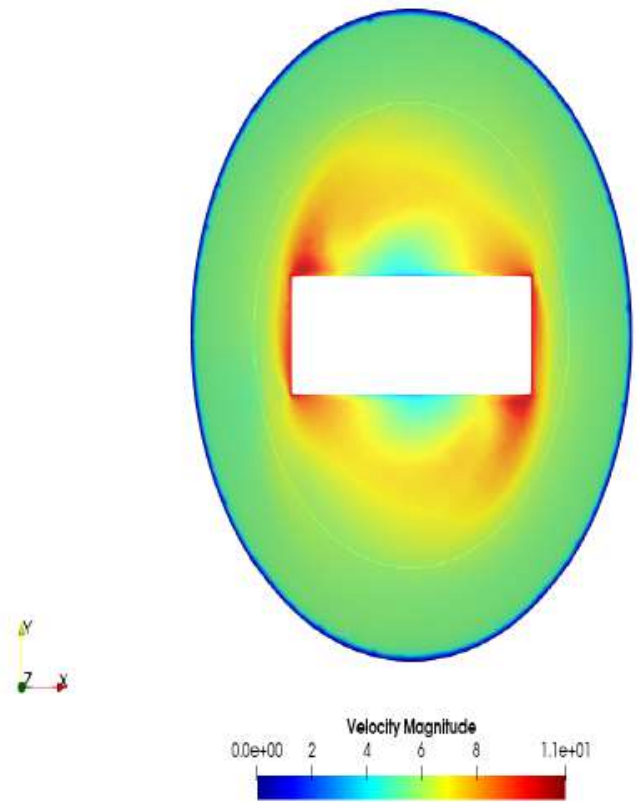

Figure 4.8: Velocity magnitude contour plot obtained from $k-\varepsilon$ realisable model in the XZ plane at $\mathrm{z}=4.5 \mathrm{~mm}$.

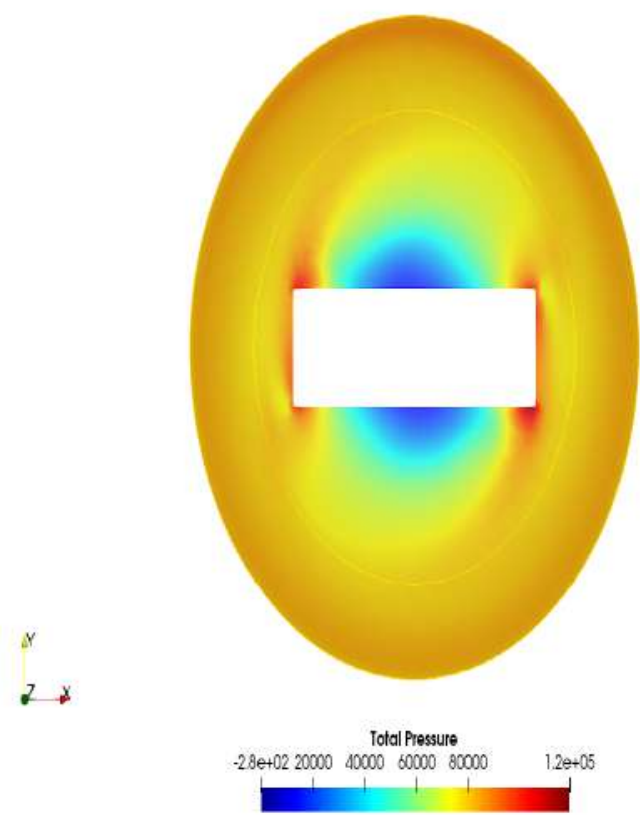

Figure 4.9: Total pressure contour plot obtained from $k-\varepsilon$ realisable model in the $\mathrm{XZ}$ plane at $\mathrm{z}=4.5 \mathrm{~mm}$. 


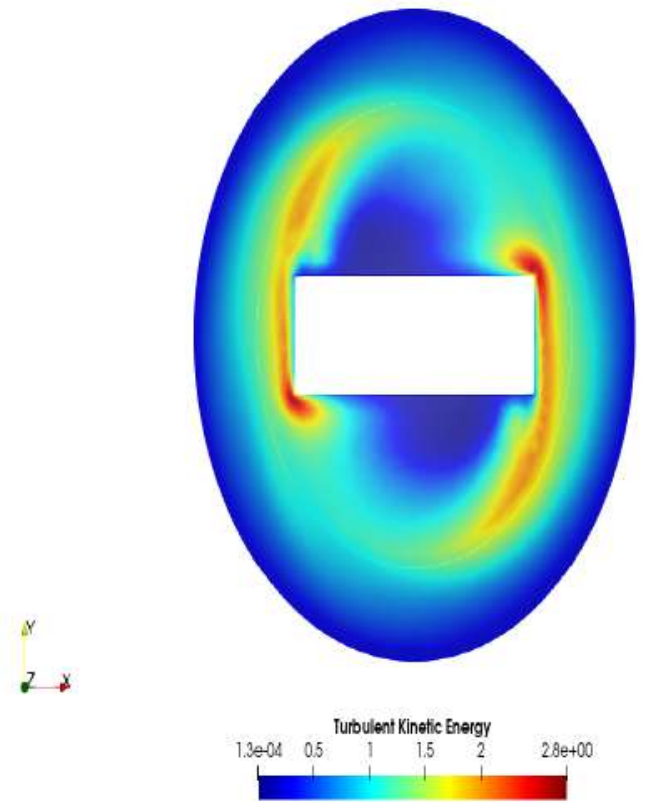

Figure 4.10: Turbulent kinetic energy contour plot obtained from $k-\varepsilon$ realisable model in the XZ plane at $\mathrm{z}=4.5 \mathrm{~mm}$.

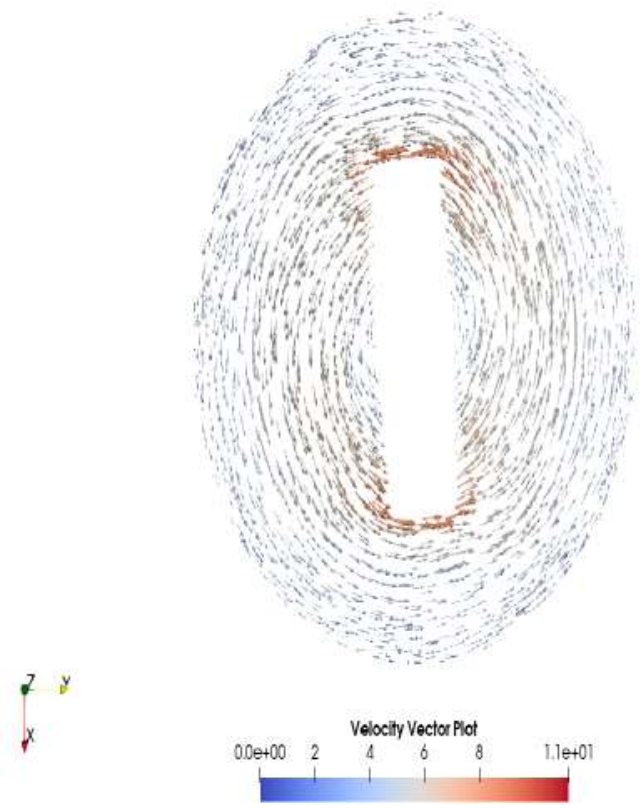

Figure 4.11: Velocity vector plot obtained from $k-\varepsilon$ realisable model in the XZ plane at $\mathrm{z}=4.5 \mathrm{~mm}$. 
the $k-\varepsilon$ models. This means that all boundary conditions for wall function mesh refer to the approach of the wall function, while the fine mesh of the appropriate Reynolds number boundary conditions is applied. Figures (4.12), (4.13) and (4.14) show the contours of velocity, total pressure and turbulent kinetic energy contours of the $k-\omega$ SST model used in the current research.

From the contour plots of velocity and pressure, it was observed that the $k-\omega$ SST model accurately predicted results near the walls of the magnetic zone, but over-predicted or under-predicted results at locations far from the boundary layer. On the other hand, the $k-\varepsilon$ model sometimes overestimated results near the boundary layer.

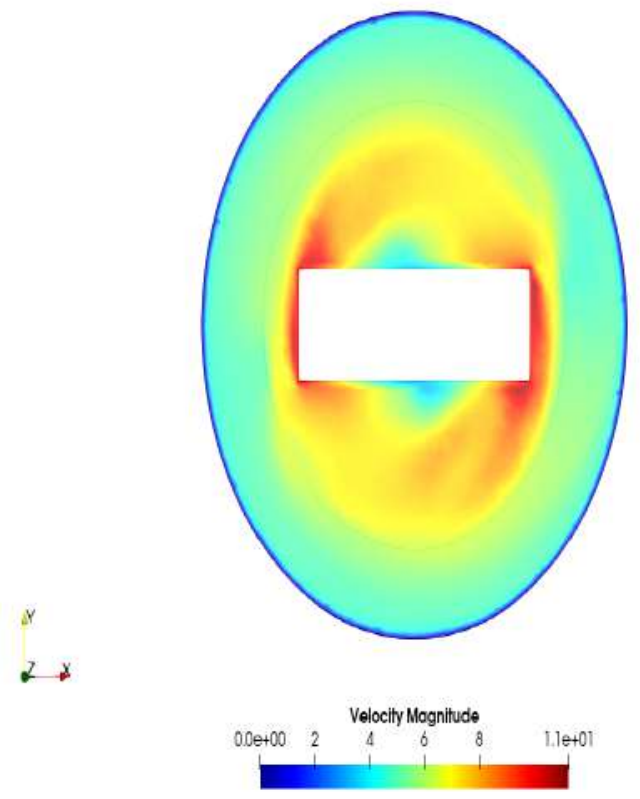

Figure 4.12: Velocity magnitude contour obtained from $k-\omega$ SST model in the $\mathrm{XZ}$ plane at $\mathrm{z}=4.5 \mathrm{~mm}$.

Two vortical roll ups were observed near the magnet in the circulation zone at a higher rotational speed as shown in the figure (4.15). These regions were found to have a maximum amount of turbulent kinetic energy dissipation. Phase 2 Volume of Fluid (VOF) contours for the both the turbulence models are depicted in figures (4.16) and (4.17). More enhanced fluid mixing was observed when the 


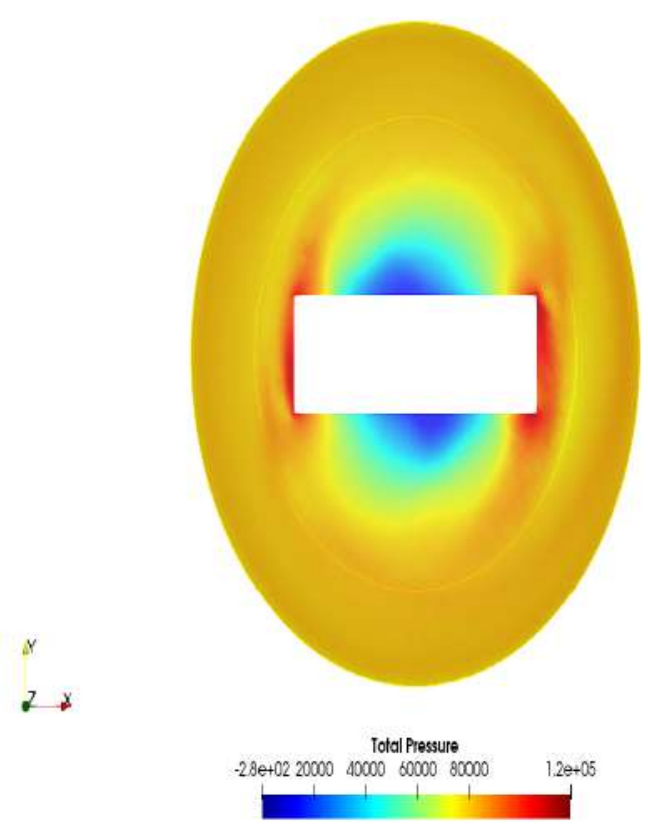

Figure 4.13: Total pressure contour obtained from $k-\omega$ SST model in the XZ plane at $\mathrm{z}=4.5 \mathrm{~mm}$.

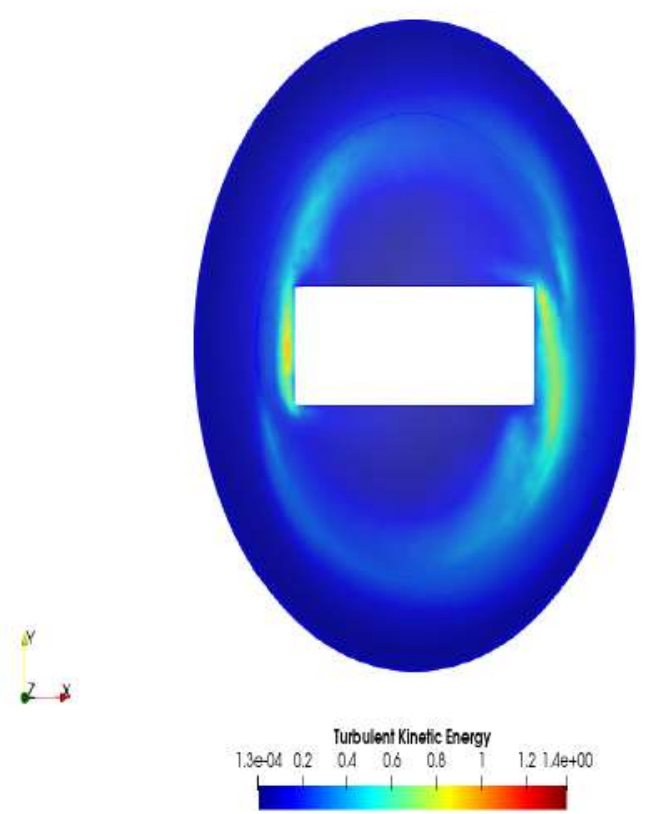

Figure 4.14: Turbulent kinetic energy contour obtained from $k-\omega$ SST model in the XZ plane at $\mathrm{z}=4.5 \mathrm{~mm}$. 


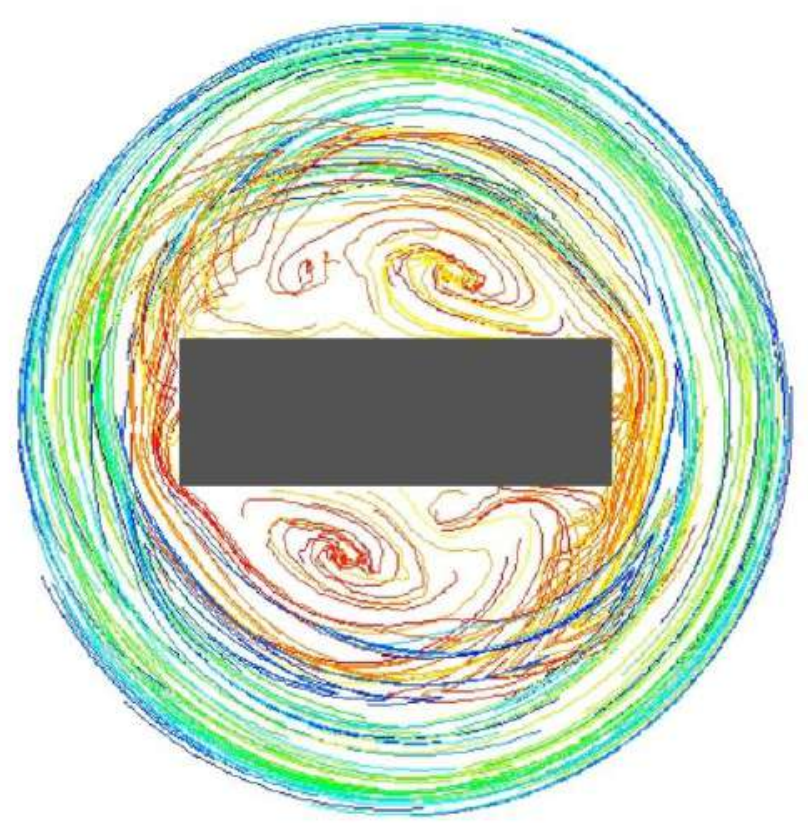

Figure 4.15: Streamline plot obtained from $k-\omega$ SST model in the XZ plane at $\mathrm{z}=4.5 \mathrm{~mm}$.

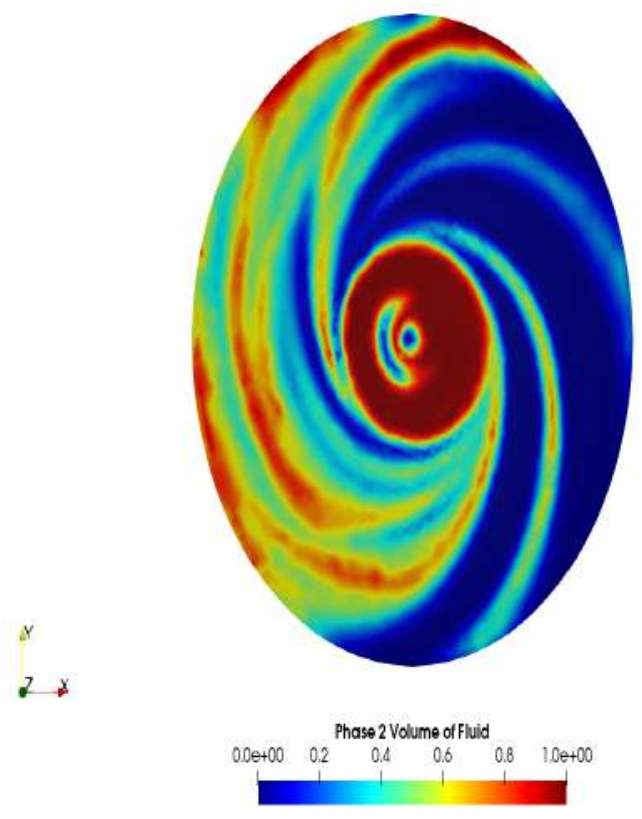

Figure 4.16: Phase 2 volume of fluid contour obtained from $k-\omega$ SST model. 


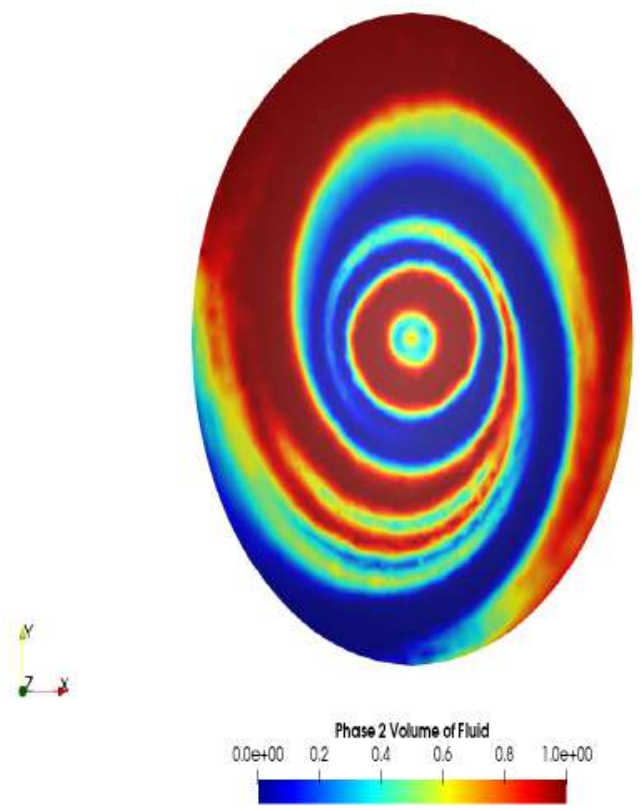

Figure 4.17: Phase 2 volume of fluid contour obtained from $k-\varepsilon$ realisable model.

$k-\varepsilon$ model was used compared to the $k-\omega$ model. The former model overpredicted the results close to the boundary layer, but it was in good agreement to predict results far from the boundary zone of the magnets.

\subsection{Summary}

The Computational Fluid Modeling of the fluid inside the lysing chamber was discussed in this chapter. Two different Reynolds Averaged Navier Stokes (RANS) turbulence models were compared with each other and the obtained results were validated with the case study described in this chapter. Another contribution through this chapter was the development and numerical analysis of the lysing chamber in order to understand the fluid-structure interaction and to help in the process of design optimization of the microfluidic chip.

The experimental validation of the automated $\mu$-MAMA and the A-NECS are presented in the following chapter. The results of the CFD modeling are also described. 


\section{Chapter 5}

\section{Results and Discussion}

In order to test whether the $\mu$-MAMA and A-NECS systems will be able to perform the desired task, a series of experimental tests were carried out and key points were documented that could possibly lead to the failure of instrumentation suite and are discussed here in conjunction with the experimental results in section 5.1. Finally, section 5.2 details the result obtained from the CFD simulations.

\subsection{Experimental Results}

The environmental sample to be tested was collected from sites considered to be a significant analogous astrobiology site for possible habitats currently present on Mars and the Europa and Enceladus cold moons. Samples were collected by the McGill Research Team during a field trip to the McGill Arctic Research Station (MARS) in July 2019 from different locations using an astrobiological drill designed by a student of the Carleton Space Exploration Research Group under the guidance of Professor Ellery. Before the field test, a series of experimentation was performed by the McGill research team in their laboratory to identify the dyes to be used for the microbial activity detection. After the experiments, two different dyes namely, Alamar dye Reagent and Biolog dye were chosen to for analysis as they were able to detect activity at a level as low as 100 cells/well. All experiments were performed in situ with an integrated automated prototype 
developed as a part of this research. A summary of the experiments carried out are shown in table (5.1) and an assembly of the instrumentation suite is shown in figure (5.2).

Table 5.1: Summary of the tests performed for the $\mu$-MAMA unit.

\begin{tabular}{|c|c|c|c|c|}
\hline $\begin{array}{c}\text { Sample Collection } \\
\text { Site }\end{array}$ & Test Case & Redox Dye & $\begin{array}{c}\text { Desired Temperature } \\
\left({ }^{\circ} \mathrm{C}\right)\end{array}$ & Microbial Activity \\
\hline \multirow{2}{*}{ Cryoconite Hole } & Case 1 & Alamar dye & \multirow{2}{*}{$12{ }^{\circ} \mathrm{C}$} & Positive \\
\cline { 2 - 3 } & Case 2 & Biolog dye & & \multirow{2}{*}{ Positive } \\
\hline \multirow{2}{*}{ Gypsum Hill Spring } & Case 1 & Alamar dye & \multirow{2}{*}{$12{ }^{\circ} \mathrm{C}$} & \multirow{2}{*}{ Negative } \\
\cline { 2 - 3 } & Case 2 & Biolog dye & & \\
\hline \multirow{2}{*}{ Lost Hammer Spring } & Case 1 & Alamar dye & \multirow{2}{*}{$12{ }^{\circ} \mathrm{C}$} & \\
\cline { 2 - 3 } & Case 2 & Biolog dye & &
\end{tabular}

Two cores and one dilution of the microbial mat from the springs of Gypsum Hill were extracted using the drill and are as follows:

1. One sediment core from the Lost Hammer Spring, and

2. One glacial ice core from Cryoconite Hole.

\subsection{1 $\mu$-MAMA Device Analysis}

\subsubsection{Experimental Setup}

The $\mu$-MAMA sub-unit comprised two peristaltic pumps, two solenoid valves, one membrane filter, two Falcon tubes, one temperature control chamber, one splitter manifold, PTFE tubing and the micro assay. One of the peristaltic pumps with a flow rate of $40 \mu l$ was mounted outside the temperature control chamber. The purpose of this pump was to transfer the fluid from the sample collected in the heating pot to one of the Falcon tubes placed inside the chamber. In this research, $50 \mathrm{ml}$ Falcon tubes were used to store the dye reagent and temporarily store the environmental sample to be tested. Another peristaltic pump was used to transfer 
the fluid temporarily stored in one of the Falcon tubes to the microassay and also to transfer the Alamar blue dye reagent in a sequence of steps. Before the sample was transferred to the microassay the sample was filtered through a 0.20 $\mu m$ membrane filter as shown in figure (5.1) to remove contaminants.

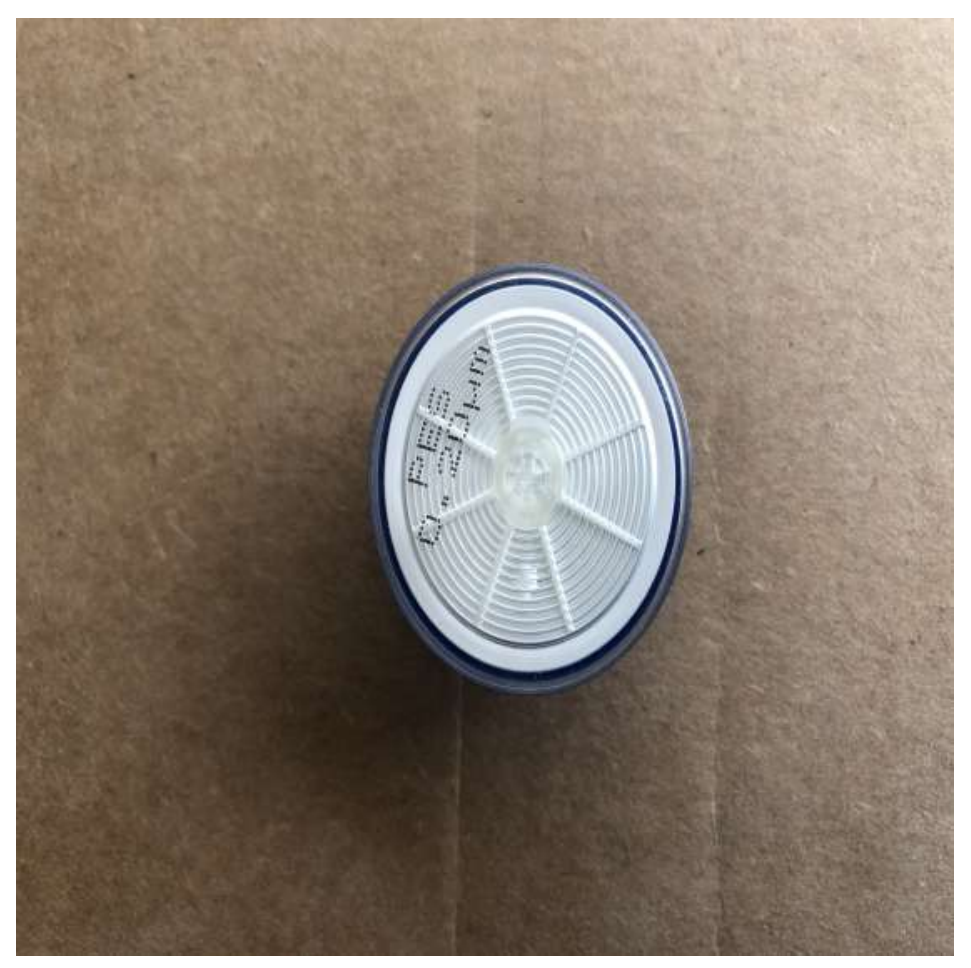

Figure 5.1: $0.20 \mu \mathrm{m}$ membrane filter used for filtration stage; scale: $30 \mathrm{~mm}$ (height) $\times 30 \mathrm{~mm}$ (diameter) .

\subsubsection{Results: Glacial Ice and Cryoconite Hole}

First Test Case: The sub-unit was placed in a temperature controlled chamber and the desired temperature was set to $12^{\circ} \mathrm{C}$. Prior to the field tests, the $\mu$-MAMA was sealed using a silicone rubber sealant and a blank run was performed. Figure (5.3) shows the algorithm used for automating the process. The experiment was performed in the following sequence. In the first place, the environmental sample was collected by automated drill and then the sample was passed through the heating cup, which consisted of a filtration stage. The main objective behind the use of a melting pot was to ensure that the sample that entered into the microfluidic system was in a liquid state for biochemical analysis. A peristaltic 


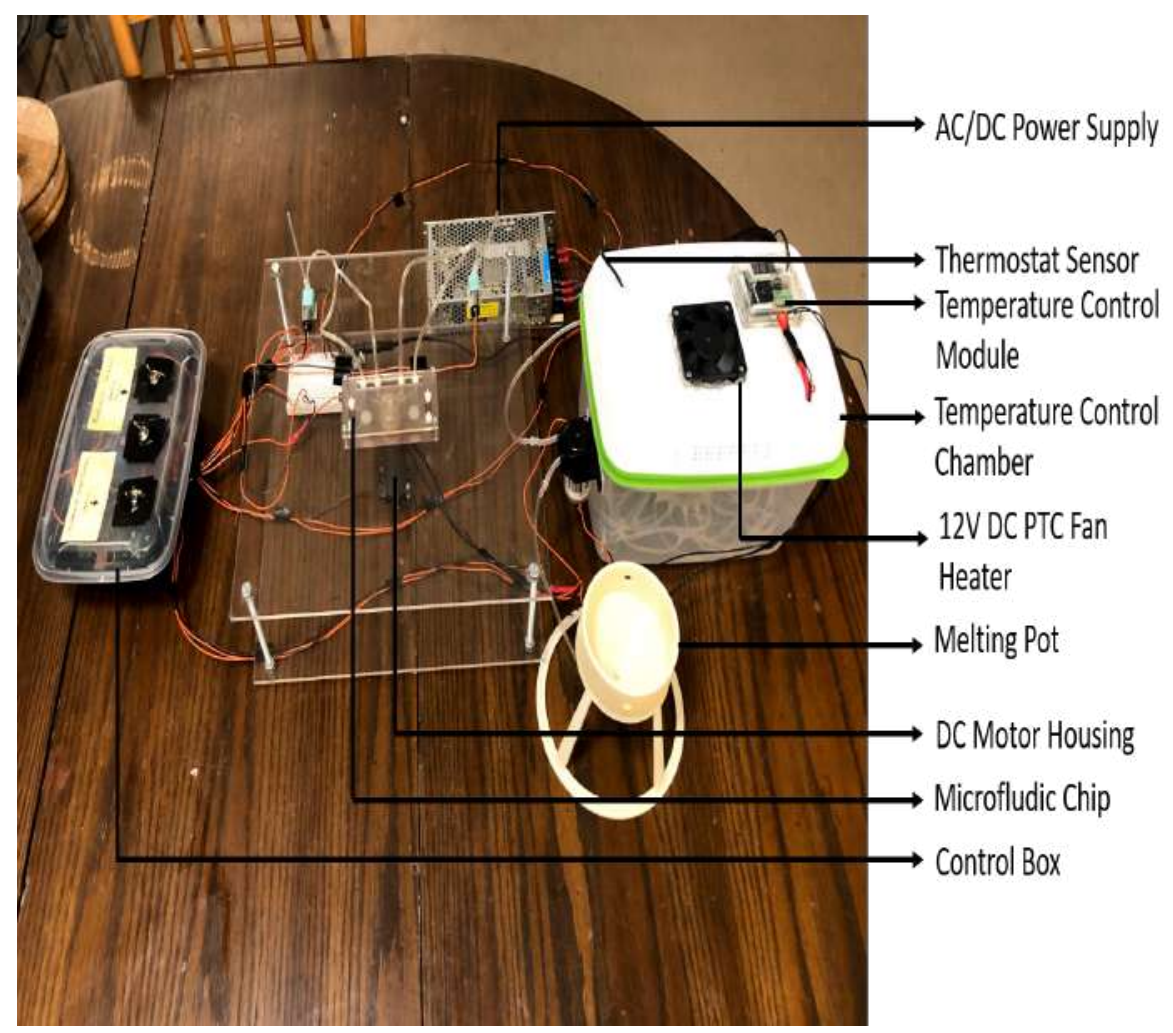

Figure 5.2: Assembly of the instrumentation suite; scale: $35 \mathrm{~cm} \times 31 \mathrm{~cm} \times 17$ $\mathrm{cm}$.

pump was used to transfer the environmental sample to the falcon tubes. After the sample was temporarily stored in one of the Falcon tubes, Solenoid Valve1 (SV1) and MicroPump2 (MP2) were turned on. With the help of micropump2, $5 \mathrm{ml}$ of the stored sample was moved to the microassay through the splitter manifold. Once the sample reached the wells of the assay, the Solenoid Valve (SV1) and Micropump2 (MP2) were switched off as indicated in the flowchart. After SV1 and MP2 were switched off, the MP2 and SV2 were switched on so that $10 \mu \mathrm{l}$ of dye reagent stored in the other Falcon tube was transferred to the $\mu$-MAMA via the circuitry. Each well of the microassay was filled with $90 \mu l$ of environmental sample and $10 \mu l$ of dye reagent. Finally, all the instruments were turned off and the solution was left to react for 8-10 hours and microassay was constantly monitored for microbial activity.

Upon completion of the reaction time, microbial activity was detected as the dye present in some wells changed its colour from blue to red, and in the remaining wells from blue to translucent, indicating the presence of microbial organisms in 


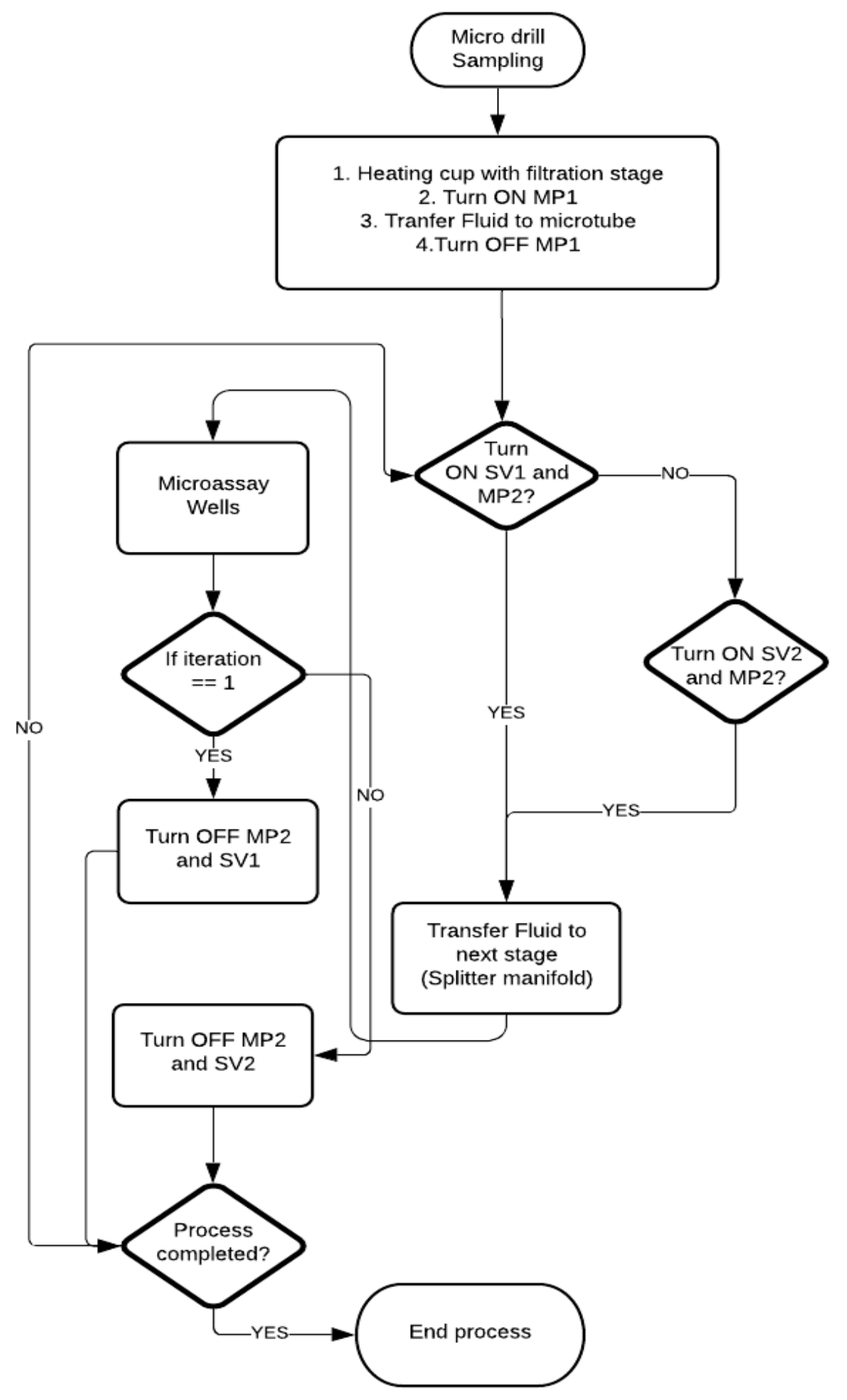

Figure 5.3: A flowchart of the simulation algorithm. 
the sample.

Second Test Case: Before the test was carried out for the second case of the sample, the old microassay was replaced with a new microassay and a rinse step was performed to clean the tubes and remove the contaminants. After completion of the cleansing procedure, the desired temperature was set to $25^{\circ} \mathrm{C}$ and a similar procedure was followed as discussed in the first case. This time, instead of using Alamar blue dye reagent, Biolog EcoPlate reagent was used to check for microbial activity. Following the completion of the reaction, microbial activity was detected in the sample as the microorganisms present in the sample oxidised the provided substrate, resulting in a change in colour of the dye.

\subsubsection{Results: Gypsum Hill Springs}

Two similar test cases were conducted with samples collected from Gypsum Hill Springs for the detection of microbial activity. After confirming the presence of microbial activity in the sample collected from Cryoconite Hole, the microassay was replaced by a new microassay and the rinse step was carried out. Following completion of the rinse step, similar procedures were followed, as discussed in the first and second Cryoconite Hole Test Cases, for two different dyes under their respective inoculation conditions. Similar behaviour was observed as in the previous cases which demonstrated the presence of the micro-organisms in the sample.

\subsubsection{Results: Lost Hammer Springs}

After the activity was successfully detected in the first two samples, two additional tests were performed with the sample collected from Lost Hammer Spring. However, no activity was observed in the sample this time. This could have happened for the following reasons:

1. There were fewer cells in the sample, possibly less than the redox dye detection limit chosen. 
2. Perhaps the microorganism present in the sample has not yet been discovered and therefore the chosen dye was not ideal in this case.

\subsubsection{A-NECS Device Analysis}

\subsubsection{Experimental Set up}

In order to test the Automated Nucleic Acid Extraction System (A-NECS), a permanent Neodymium Plated block magnet with a length of $19.05 \mathrm{~mm} \times 6.35$ $\mathrm{mm}$ in width $\times 8.37 \mathrm{~mm}$ in height with radial magnetisation was placed inside the lysing chamber. The base microfluidic chip was bonded to the top plate and a silicone seal was placed between the two plates to ensure that there was no sample loss during operation and to prevent contamination. The block magnet was now coupled to a disc magnet of $19.5 \mathrm{~mm}$ in diameter and $6.35 \mathrm{~mm}$ in thickness. For the separation between the rotating disc magnet and the bottom of the chip, two clear and UV-resistant cast acrylic sheets with a thickness of $3.175 \mathrm{~mm}$ were used. The distance between the bottom of the chip and the rotating magnet was kept at $5 \mathrm{~mm}$ and the disc magnet was mounted on the magnet housing as shown in figure (5.4). The magnet housing was then coupled to a $2 \mathrm{~mm}$ diameter DC motor shaft. The specification of the DC motor used is shown in the table (5.2). A motor housing was designed to house the DC motor as depicted in figure (5.5). It consisted of $6 \times \mathrm{M} 2$ through holes at the top positioned at an angle of $60^{\circ}$ in a circular pattern to screw the motor in the housing. The motor housing was mounted on the bottom sheet of acrylic with $6 \times$ M3 nuts and bolts. Finally, the microfluidic chip was connected to the peristaltic pump to transfer the fluid inside the lysing chamber via 10-32 UNF single barb tight seal fittings. Another peristaltic pump was connected to the circuitry to transport the fluid to the reservoirs on the microfluidic chip. The flow rate of the micropump was computer controlled to adjust the flow rate as required.

While the sample was flowing through the system, the block magnet was rotating due to the magnetic field rotation caused by the disc magnet coupled to 


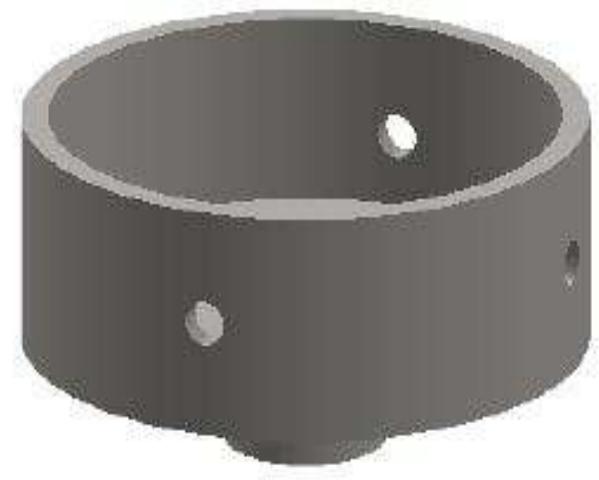

Figure 5.4: 3D model of the magnet housing; scale: $9.85 \mathrm{~mm}$ (height) $\times 21.10$ $\mathrm{mm}$ (diameter).

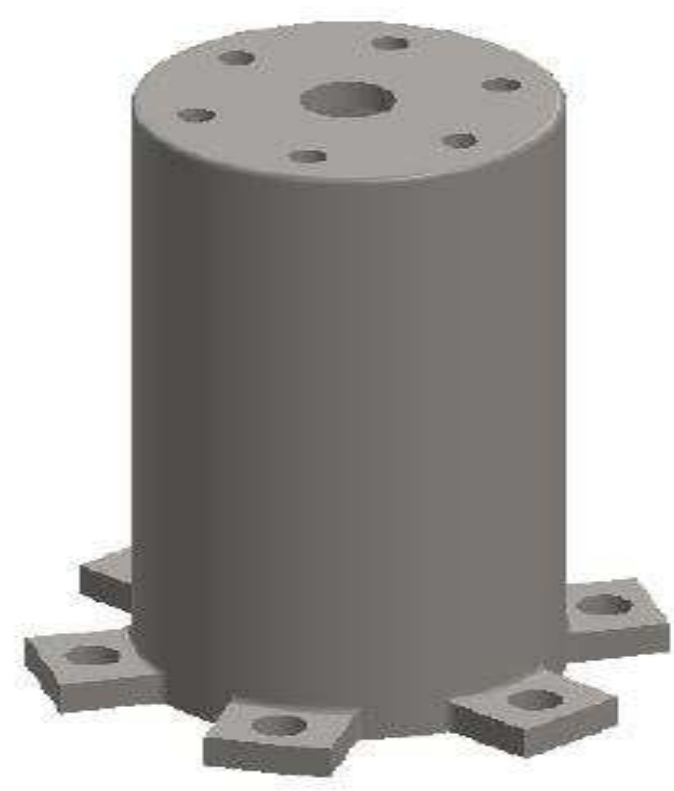

Figure 5.5: 3D model of the DC motor housing; scale: $78 \mathrm{~mm}$ (height) $\times 23.50$ $\mathrm{mm}$ (diameter). 
Table 5.2: Brushed DC motor attributes.

\begin{tabular}{|c|c|}
\hline Type & Description \\
\hline Rated Voltage & $12 \mathrm{~V} \mathrm{DC}$ \\
\hline RPM & $10000 \mathrm{rpm}$ \\
\hline Shape/Dimensions & Round $-22 \mathrm{~mm}$ diameter \\
\hline Shaft diameter & $2 \mathrm{~mm}$ \\
\hline Operating Temperature & $-30^{\circ} \mathrm{C} \mathrm{to} 85^{\circ} \mathrm{C}$ \\
\hline
\end{tabular}

the DC motor shaft. As a result, an adjustable speed lysing chamber capable of disrupting the cell walls in continous flow was achieved. The lysate product was recovered using a peristaltic pump and stored in a tube. Two microfluidic chips were tested with this set up and are discussed below.

\subsubsection{Results: Microfluidic Chip with Baffle}

In order to check that the introduction of a baffle in the lysing chamber would have a direct impact on the yield of the lysate or not, a microfluidic baffle chip (see figure (3.22)) was tested under similar conditions as described in section 5.1.2.1. Due to the lack of availability of the environmental sample collected by the McGill research team, the model was tested on a sample collected from Hogs Back Lake in Ottawa. From the experimental results, it was observed that the magnet was not able to rotate freely due to baffles, which resulted in poor mixing and therefore decreased the chances to recover high yield lysate. The following test was performed with a microfluidic chip without baffle to mitigate the current problem.

\subsubsection{Results: Microfluidic Chip without Baffle}

The microfluidic setup of A-NECS was replaced with the new microfluidic chip without baffle and tested under similar conditions. From the experimental results 


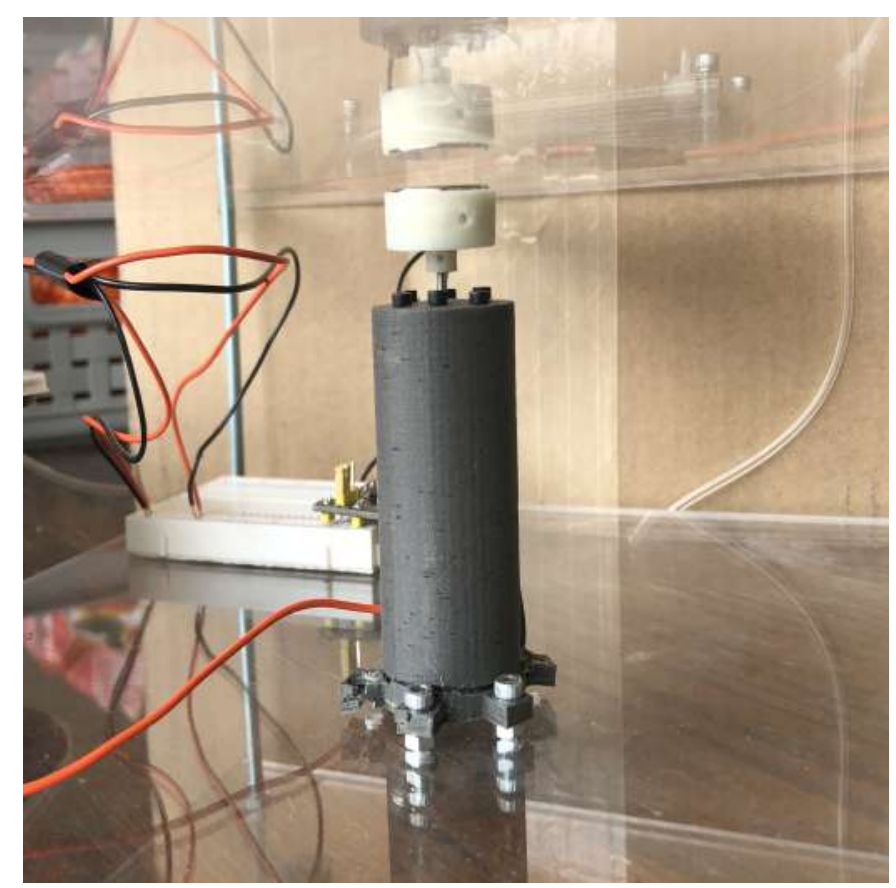

Figure 5.6: Assembly of the disc magnet coupled with DC motor shaft; scale: $83 \mathrm{~mm}$ (height) $\times 23.50 \mathrm{~mm}$ (diameter).

it was observed that the block magnet was able to rotate freely when a high voltage pulse was provided to the circuit. This improved the mixing process and at the same time increased the chances of extracting a lysate with higher yield.

\subsection{Computational Fluid Dynamics Results}

Simulations were performed in six different runs and the specification of each run is given in the table (5.3). Figures (5.7), (5.8) and (5.9) show the cumulative energy dissipation curves for run 1, 2 and 3 at fluid 1 and fluid 2 for the magnet zone. Zone boundaries were determined on the basis of the turbulent kinetic energy dissipation rate gradient. The magnetic zone started from the tip of the magnet to the point where the energy dissipation gradient began to decrease. The remaining fluid was in the circulation area.

A clear break was observed at the slope of each of these curves and this break point is known as the energy dissipation cut-off rate of $\varepsilon_{c u t}$ which identified the boundary of the compartments. The first compartment was a small area around the magnet stirrer, characterised by a large, turbulent dissipation rate of kinetic 
energy. The second compartment was a very large circulation region where the turbulent flow field was almost homogeneous and the turbulent kinetic energy dissipation rate was very small.

Vortical rolls up were also observed in these zones as shown in figure (4.15). Since the test was simulated at a very high speed, no significant changes in the turbulent kinetic energy of the two regions were observed.

Table 5.3: Summary of the different run specifications.

\begin{tabular}{|c|c|c|c|c|}
\hline Run Number & $\begin{array}{c}\text { Agitation Rate } \\
\qquad(\mathrm{rpm})\end{array}$ & $\begin{array}{c}\text { Turbulence } \\
\text { Model }\end{array}$ & $\begin{array}{c}\text { Density } \rho_{w} \\
\left(k g / m^{3}\right)\end{array}$ & $\begin{array}{c}\text { Density } \rho_{d} \\
\left(\mathrm{~kg} / \mathrm{m}^{3}\right)\end{array}$ \\
\hline Run 1 & 6000 & \multirow{3}{*}{$k-\varepsilon$ realisable } & 998.2 & 1250 \\
\hline Run 2 & 8000 & & 998.2 & 1250 \\
\hline Run 3 & 10000 & & 998.2 & 1250 \\
\hline Run 4 & 6000 & \multirow{3}{*}{$k-\omega \mathrm{SST}$} & 998.2 & 1250 \\
\hline Run 5 & 8000 & & 998.2 & 1250 \\
\hline Run 6 & 10000 & & 998.2 & 1250 \\
\hline
\end{tabular}

From the plots it was observed that rate of energy dissipation increased with the rate of agitation in both zones with a smaller slope in the circulation zone. The results shows that, the energy dissipation rate increased almost 4 times in the magnet region by increasing the agitation rate from 6000 to $10000 \mathrm{rpm}$.

The effect of agitation rate was plotted for three different runs of the $k-\varepsilon$ realisable model, and it was observed that the increase in the agitation rate led to an increase in the turbulent kinetic energy everywhere in the lysing chamber. By increasing the agitator speed from 6000 to $10000 \mathrm{rpm}$, the turbulent kinetic energy increased almost 3 times in the magnetic zone and the expected increase of 2 times in the circulation zone was observed. Figures (5.10), (5.11) and (5.12) show the effect of agitation rate on turbulent kinetic energy, turbulent viscosity and energy dissipation rate. From the figure (5.11), it can be clearly seen that 


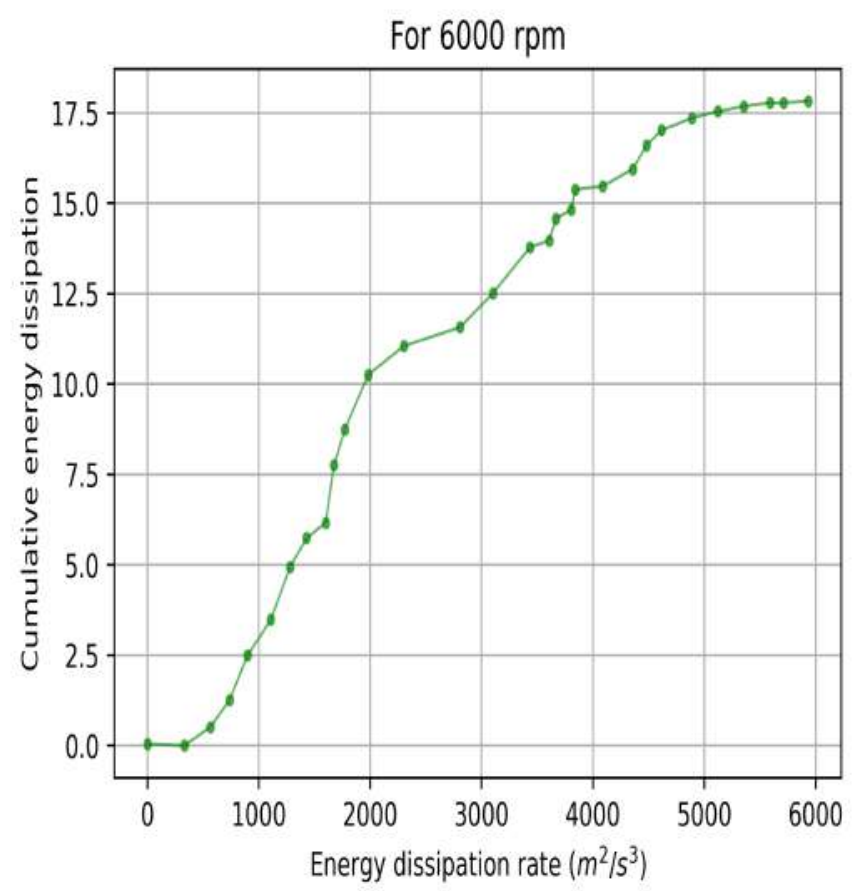

Figure 5.7: Cumulative energy dissipation for run 1 at the magnet zone.

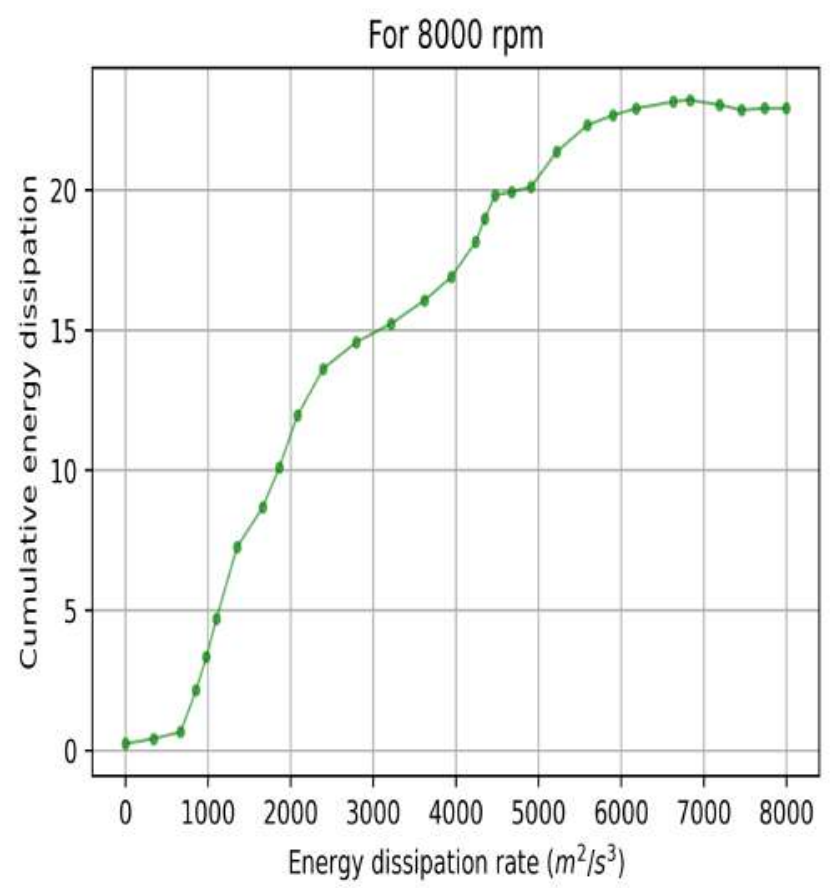

Figure 5.8: Cumulative energy dissipation for run 2 at the magnet zone. 
there has been an increase in turbulent viscosity in both zones with agitation rates. However, its was observed to be higher in the circulation zone. In circulation zone, the formation of eddies was the main contributor to the scalar transfer. In the circulation zone, therefore, the turbulent viscosity was higher than magnet zone.

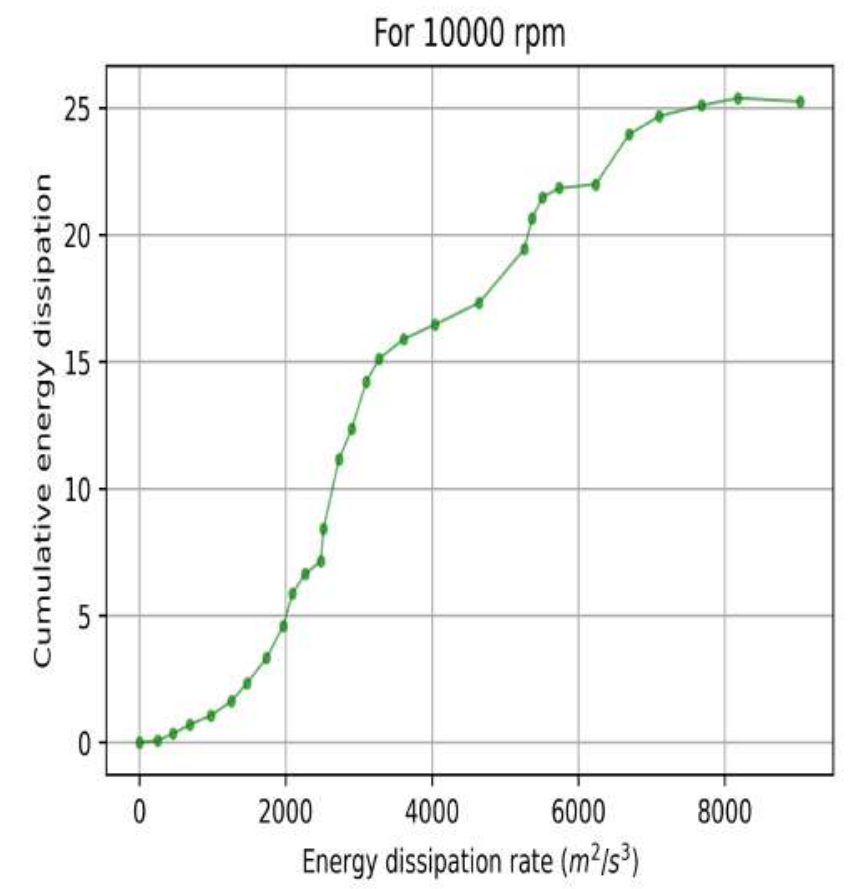

Figure 5.9: Cumulative energy dissipation for run 3 at the magnet zone.

Figure (5.13) shows the effect of agitation rate on the volume density distribution of energy dissipation. It was seen that the increase in the agitation rate lead to a shift in the peak of the volume density distribution towards higher values. With an increase in agitation rate from 6000 to $10000 \mathrm{rpm}$, the volume density distribution peak shifted from $2258.96 \mathrm{~m}^{2} / \mathrm{s}^{3}$ to $7360.57 \mathrm{~m}^{2} / \mathrm{s}^{3}$, that is, about the third order of magnitude. As discussed above, increasing the rate of agitation lead to 4-fold increase in energy dissipation rate, while turbulent kinetic energy increased by 3 fold. The length scale of eddies therefore decreased by 0.5 . This was calculated from the formula used by [123], which stated that length scale of eddies is related to turbulent properties through the correlation:

$$
L \propto \frac{(k)^{\frac{2}{3}}}{\varepsilon}
$$




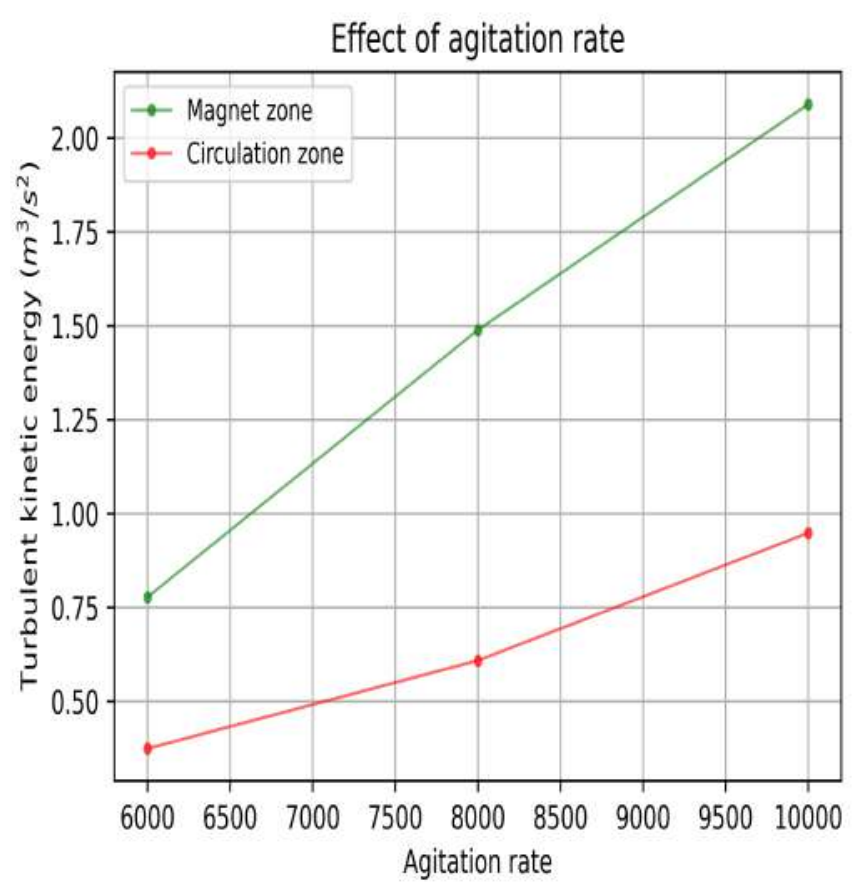

Figure 5.10: Effect of agitation rate on the turbulent kinetic energy for $k-\varepsilon$ realisable model.

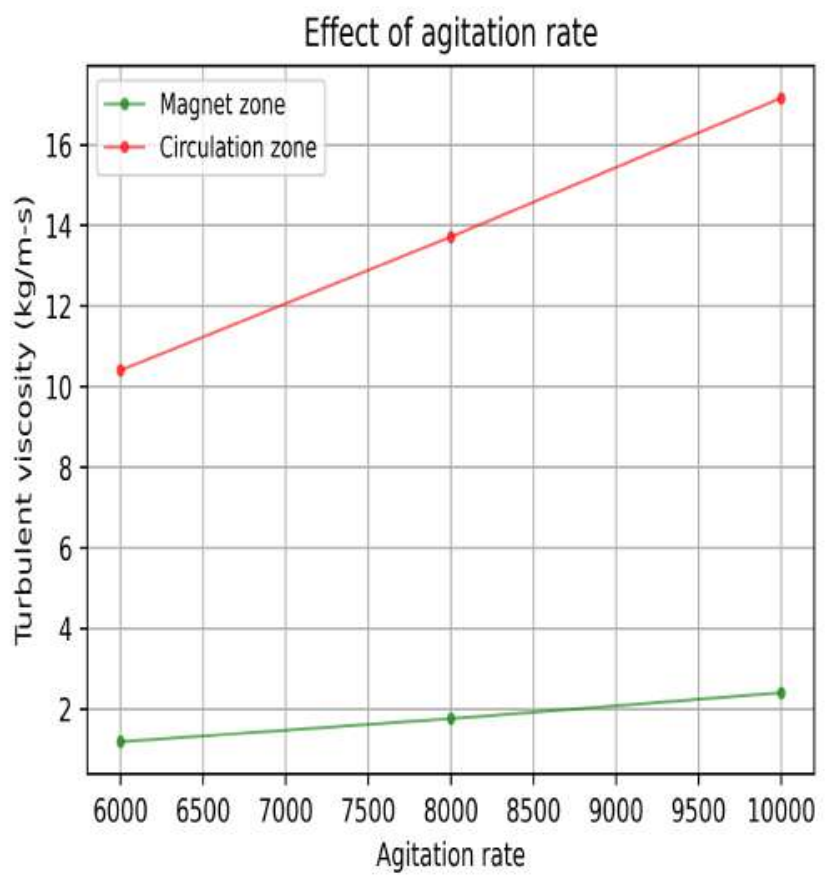

Figure 5.11: Effect of agitation rate on the turbulent viscosity for $k-\varepsilon$ realisable model. 


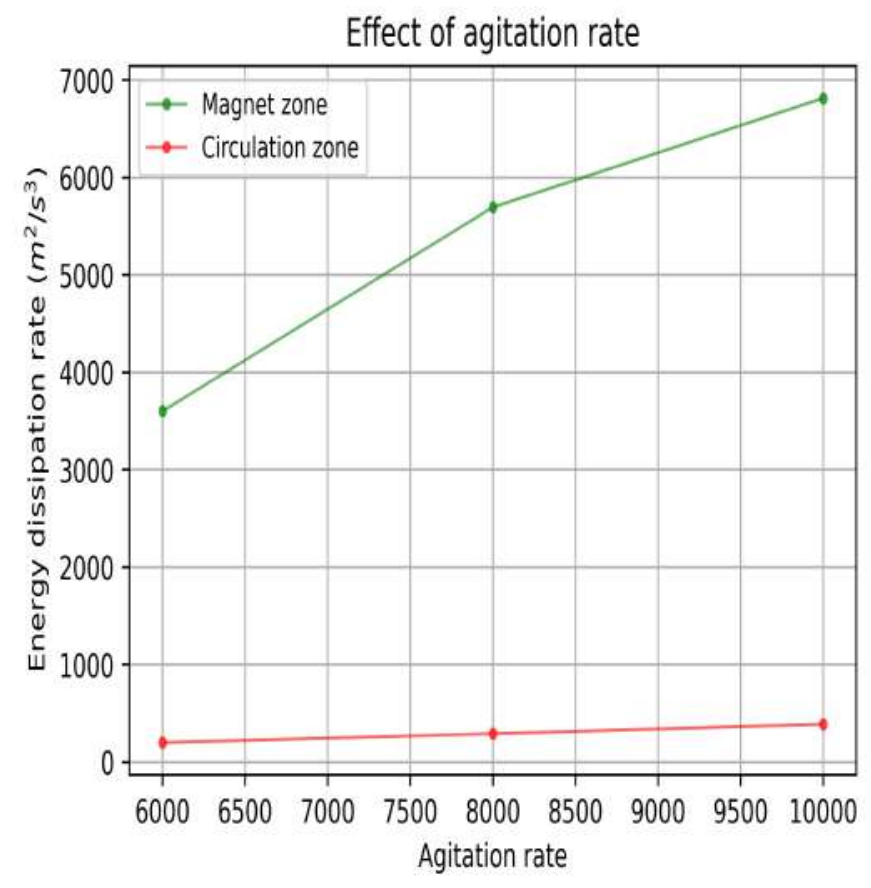

Figure 5.12: Effect of agitation rate on the energy dissipation rates for $k-\varepsilon$ realisable model.

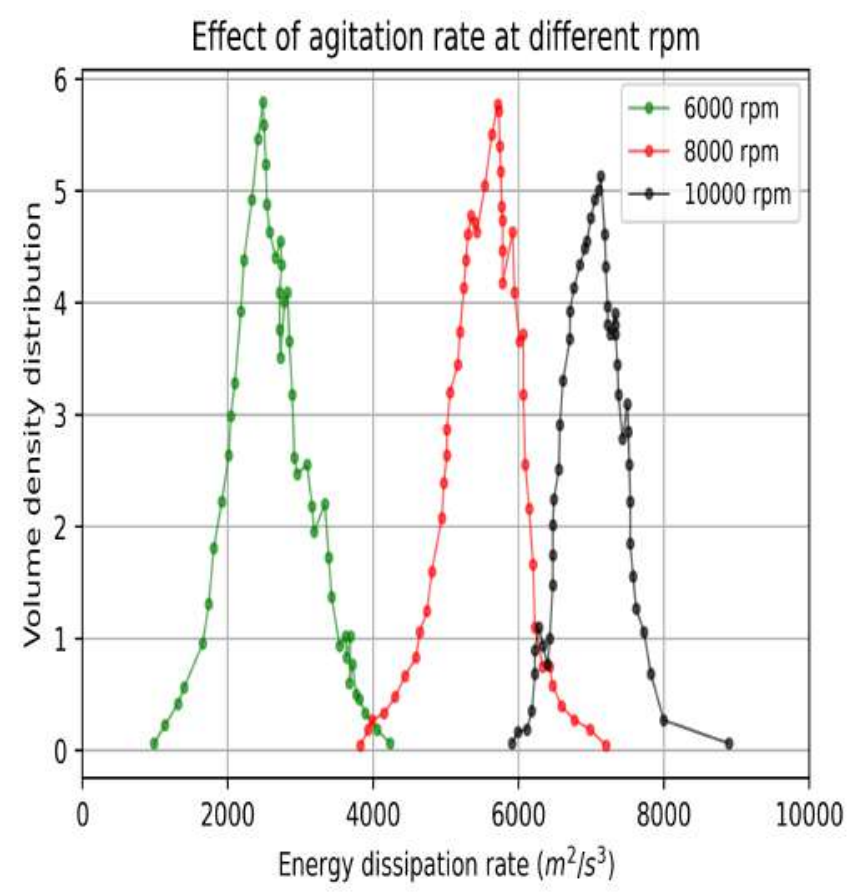

Figure 5.13: Plot of volume density distribution of the energy dissipation rates for different agitation rates of $k-\varepsilon$ realisable model.

As a result, an increase in the agitation rate resulted in a decrease in length scale of eddies, as expected from the calculations. It is important to note that 
smaller values of the length scale eddies are associated with higher values of turbulent intensity and energy dissipation. Henceforth, as the agitation rate increased, the values of energy dissipation rate shifted to higher values as described above. Finally, Alexopoulous et al. [119] stated that the average distribution varies proportionally to the third power of the agitation rate, which was also seen in this research. This was an indication that the $k-\varepsilon$ realisable model produced better results compared to the $k-\omega$ SST model as the former produced the averaged energy dissipation rate values that were qualitatively valid. 


\section{Chapter 6}

\section{Conclusion and Future Work}

\subsection{Conclusion}

The main objectives of this thesis were to:-

1. Develop an automated end-to-end analysis system capable of performing tests in an astrobiological environment without any human intervention.

2. A mathematical model to predict flow patterns through the channels.

3. Numerical study of the lysing chamber in order to optimize the design of the microfluidic chip.

4. Perform a series of experimental test to validate the developed prototype.

The key contributions of this thesis were:-

1. Development of the novel $\mu$-MAMA prototype with:-

- an advanced sealing system to eliminate cross contamination

- 16 fully independent wells with each well having its own inlet and outlet to test multiple samples in a single assay

2. Development of the lysing chamber, a completely novel system for the automated extraction of nucleic acids from environmental samples that can be fed to MinION for detection 
3. Numerical analysis of the lysing chamber in the microfluidic chip to optimize the design model and increase the recovery of lysate produced.

4. A portion of this research has been presented and published in the proceedings of the IAC 2019 Conference [23]

The first objective of this thesis, the development of an end-to-end analysis system, was accomplished by designing a control algorithm capable of performing all operations in an automated manner with precision. The model was validated with a series of experimental tests that resulted in an advanced $\mu$-MAMA and A-NECS platform capable of detecting microbial activity in the environmental sample and recovering high yield lysate for nucleic acid detection.

The second objective was to establish a mathematical model to predict the flow patterns through the channels. This was achieved by a means of a detailed review of the existing literature available and by comparison to the current model. The literature review provided the best foundation to develop the 3D model with a minimum error and to choose the appropriate instrumentation for the integration of the life detection system. A range of experimental tests were carried out to ensure that the values obtained from modelling were in close relation to the experimental findings. The instrumentation used to integrate the system was found to be an improvement over the pre-existing systems and hence have a potential to be integrated into future astrobiology space missions. On the other hand, preexisting systems remained high in mass, high in operational cost, large in size, and high in energy requirements. Such instruments were completely unsuitable for missions to locations such as Mars, Europa or Enceladus.

The third objective was to perform CFD simulations of the lysing chamber in the microfluidic chip and to highlight regions of maximum energy dissipation, turbulent kinetic energy, turbulent kinetic viscosity, effect of agitation rate and also to understand the fluid-structure interaction. The results showed that the small region around the magnetic stirrer had the maximum turbulent kinetic energy dissipation. Vortical rolls up near the magnetic zone were observed and the effect 
of agitation rate on the length scale of the eddies formation near the magnetic zone was studied. From the calculations, it was found that length of eddies decreased by 0.5 as the agitation rate increased. However, turbulent kinetic energy was found to be higher in both identified zones. Finally, the volume density distribution of the energy dissipation rate was concluded to be an important parameter, as it presented a better representation of the dissipated energy rate values in the selected regions over a simple list of the discrete energy dissipation rate values.

\subsection{Future Work}

The platforms and methods illustrated here are highly portable and offer the possibility to investigate microbial ecology in real-time, complex and remote environments. Future planetary exploration is not likely not rely on a single instrument, rather a set of instruments will have to be used in conjunction to confirm whether extant life existed on such bodies. While Nanopore MinION sequencing technology has significant potential as an unambiguous biosignatures detection platform for future planetary exploration missions, a number of hurdles remain. Further work needs to be done for optimising these tools for a flight mission, such as;

- Perform multiple end-to-end experimental tests to improve the robustness of both $\mu$-MAMA and A-NECS systems.

- Further miniaturisation of the instrumentation suite so that it could be integrated into the Kapvik Rover designed by the Carleton space exploration research group or into small mission concepts from micro-penetrator to nanorovers.

- A more detailed CFD analysis considering parameters such as different magnet size, amount of fluid in the lysing chamber and viscosity effect.

- Conduct field tests with the cryo-environment samples on the A-NECS system to improve the recovery of the lysate produced. 


\subsection{Applications}

The instruments adopted for this research are fully portable and have applications in industry for field detection and identification of micro-organisms in remote extreme environments, as well as for healthcare settings. In addition to its use in extreme cryo-environments such as Europa, Enceladus and Mars, this instrumentation system may be useful for rapid clinical diagnosis, biomonitoring, food safety and environmental analysis, including, for example, monitoring, detection and identification of pathogens during a pandemic or outbreak in remote locations. 


\section{References}

[1] M. C. Malin and K. S. Edgett, "Evidence for recent groundwater seepage and surface runoff on mars," Science, vol. 288, no. 5475, pp. 2330-2335, 2000.

[2] S. W. Squyres and A. H. Knoll, "Sedimentary rocks at meridiani planum: Origin, diagenesis, and implications for life on mars," Earth and Planetary Science Letters, vol. 240, no. 1, pp. 1-10, 2005.

[3] J.-P. Bibring, Y. Langevin, J. F. Mustard, F. Poulet, R. Arvidson, A. Gendrin, B. Gondet, N. Mangold, P. Pinet, F. Forget, et al., "Global mineralogical and aqueous mars history derived from omega/mars express data," science, vol. 312, no. 5772, pp. 400-404, 2006.

[4] L. Hays, L. Archenbach, J. Bailey, R. Barnes, J. Barros, C. Bertka, P. Boston, E. Boyd, M. Cable, I. Chen, et al., "Nasa astrobiology strategy," 2015.

[5] M. P. Golombek, "A message from warmer times," Science, vol. 283, no. 5407 , pp. $1470-1471,1999$.

[6] L. Ojha, M. Wilhelm, S. Murchie, A. McEwen, J. Wray, J. Hanley, M. Massé, and M. Chojnacki, "Spectral evidence for hydrated salts in recurring slope lineae on mars, nat," 2015.

[7] J. S. Kargel, J. Z. Kaye, J. W. Head III, G. M. Marion, R. Sassen, J. K. Crowley, O. P. Ballesteros, S. A. Grant, and D. L. Hogenboom, "Europa's 
crust and ocean: origin, composition, and the prospects for life," Icarus, vol. 148 , no. 1 , pp. 226-265, 2000.

[8] H. Melosh, A. Ekholm, A. Showman, and R. Lorenz, "The temperature of europa's subsurface water ocean," Icarus, vol. 168, no. 2, pp. 498-502, 2004.

[9] J. unter Waite Jr, "Cassini ion and neutral mass spectrometer: Enceladus plume," science, vol. 1121290, no. 1419, p. 311, 2006.

[10] C. P. McKay, C. C. Porco, T. Altheide, W. L. Davis, and T. A. Kral, "The possible origin and persistence of life on enceladus and detection of biomarkers in the plume," Astrobiology, vol. 8, no. 5, pp. 909-919, 2008.

[11] L. Roth, K. D. Retherford, J. Saur, D. F. Strobel, P. D. Feldman, M. A. McGrath, and F. Nimmo, "Orbital apocenter is not a sufficient condition for hst/stis detection of europa's water vapor aurora," Proceedings of the National Academy of Sciences, vol. 111, no. 48, pp. E5123-E5132, 2014.

[12] J. Goordial, G. Lamarche-Gagnon, C.-Y. Lay, and L. Whyte, "Left out in the cold: life in cryoenvironments," in Polyextremophiles, pp. 335-363, Springer, 2013.

[13] M. Eisenstein, "An ace in the hole for dna sequencing," 2017.

[14] J. Clarke, H.-C. Wu, L. Jayasinghe, A. Patel, S. Reid, and H. Bayley, "Continuous base identification for single-molecule nanopore dna sequencing," Nature nanotechnology, vol. 4, no. 4, p. 265, 2009.

[15] S. Majd, E. C. Yusko, Y. N. Billeh, M. X. Macrae, J. Yang, and M. Mayer, "Applications of biological pores in nanomedicine, sensing, and nanoelectronics," Current Opinion in Biotechnology, vol. 21, no. 4, pp. 439-476, 2010.

[16] B. M. Venkatesan and R. Bashir, "Nanopore sensors for nucleic acid analysis," Nature nanotechnology, vol. 6, no. 10, p. 615, 2011. 
[17] G. V. Levin, "The viking labeled release experiment and life on mars," in Instruments, Methods, and Missions for the Investigation of Extraterrestrial Microorganisms, vol. 3111, pp. 146-161, International Society for Optics and Photonics, 1997.

[18] A. F. Davila, M. Skidmore, A. G. Fairén, C. Cockell, and D. Schulze-Makuch, "New priorities in the robotic exploration of mars: the case for in situ search for extant life," Astrobiology, vol. 10, no. 7, pp. 705-710, 2010.

[19] Moderator:, D. Schulze-Makuch, Participants:, J. D. Rummel, S. A. Benner, G. Levin, V. Parro, and S. Kounaves, "Nearly forty years after viking: are we ready for a new life-detection mission?," Astrobiology, vol. 15, no. 6, pp. 413-419, 2015.

[20] M. D. Brasier, O. R. Green, and N. McLoughlin, "Characterization and critical testing of potential microfossils from the early earth: the apex 'microfossil debate'and its lessons for mars sample return," International Journal of Astrobiology, vol. 3, no. 2, pp. 139-150, 2004.

[21] A. Edwards, A. R. Debbonaire, S. M. Nicholls, S. M. Rassner, B. Sattler, J. M. Cook, T. Davy, A. Soares, L. A. Mur, and A. J. Hodson, "In-field metagenome and 16s rrna gene amplicon nanopore sequencing robustly characterize glacier microbiota," BioRxiv, p. 073965, 2019.

[22] S. S. Johnson, E. Zaikova, D. S. Goerlitz, Y. Bai, and S. W. Tighe, "Realtime dna sequencing in the antarctic dry valleys using the oxford nanopore sequencer," Journal of Biomolecular Techniques: JBT, vol. 28, no. 1, p. 2, 2017.

[23] S. Sondhiya and A. Ellery, "Direct astrobiological sampling of enceladus' subsurface vents for the micro life instrument suite," vol. Technical Programme, p. 74, IAC Publications, October 2019. 
[24] G. Taylor, "Konstantinova iv, sonnenfeld g, and jennings r," Changes in the immune system during and after space flight. Adv Space Biol Med, vol. 6, pp. 1-32, 1997.

[25] J. Wilson, C. Ott, K. H. Zu Bentrup, R. Ramamurthy, L. Quick, S. Porwollik, P. Cheng, M. McClelland, G. Tsaprailis, T. Radabaugh, et al., "Space flight alters bacterial gene expression and virulence and reveals a role for global regulator hfq," Proceedings of the National Academy of Sciences, vol. 104, no. 41, pp. 16299-16304, 2007.

[26] I. Cho and M. J. Blaser, "The human microbiome: at the interface of health and disease," Nature Reviews Genetics, vol. 13, no. 4, pp. 260-270, 2012.

[27] J. M. Kinross, A. C. von Roon, E. Holmes, A. Darzi, and J. K. Nicholson, "The human gut microbiome: implications for future health care," Current gastroenterology reports, vol. 10, no. 4, pp. 396-403, 2008.

[28] G. Church, D. W. Deamer, D. Branton, R. Baldarelli, and J. Kasianowicz, "Characterization of individual polymer molecules based on monomerinterface interactions," Aug. 18 1998. US Patent 5,795,782.

[29] J. J. Kasianowicz, E. Brandin, D. Branton, and D. W. Deamer, "Characterization of individual polynucleotide molecules using a membrane channel," Proceedings of the National Academy of Sciences, vol. 93, no. 24, pp. 1377013773, 1996.

[30] M. Morey, A. Fernández-Marmiesse, D. Castineiras, J. M. Fraga, M. L. Couce, and J. A. Cocho, "A glimpse into past, present, and future dna sequencing," Molecular genetics and metabolism, vol. 110, no. 1-2, pp. 3-24, 2013.

[31] Y. Feng, Y. Zhang, C. Ying, D. Wang, and C. Du, "Nanopore-based fourthgeneration dna sequencing technology," Genomics, proteomics $\mathscr{6}$ bioinformatics, vol. 13, no. 1, pp. 4-16, 2015. 
[32] D. T. Chiu, A. J. deMello, D. Di Carlo, P. S. Doyle, C. Hansen, R. M. Maceiczyk, and R. C. Wootton, "Small but perfectly formed? successes, challenges, and opportunities for microfluidics in the chemical and biological sciences," Chem, vol. 2, no. 2, pp. 201-223, 2017.

[33] E. L. Van Dijk, H. Auger, Y. Jaszczyszyn, and C. Thermes, "Ten years of next-generation sequencing technology," Trends in genetics, vol. 30, no. 9, pp. 418-426, 2014.

[34] A. S. Mikheyev and M. M. Tin, "A first look at the oxford nanopore minion sequencer," Molecular ecology resources, vol. 14, no. 6, pp. 1097-1102, 2014.

[35] C. L. Ip, M. Loose, J. R. Tyson, M. de Cesare, B. L. Brown, M. Jain, R. M. Leggett, D. A. Eccles, V. Zalunin, J. M. Urban, et al., "Minion analysis and reference consortium: Phase 1 data release and analysis," F1000Research, vol. 4, 2015 .

[36] E. C. Hayden, "Pint-sized dna sequencer impresses first users," Nature, vol. 521, no. 7550, pp. 15-16, 2015.

[37] E. Willerslev, A. J. Hansen, R. Rønn, T. B. Brand, I. Barnes, C. Wiuf, D. Gilichinsky, D. Mitchell, and A. Cooper, "Long-term persistence of bacterial dna," Current Biology, vol. 14, no. 1, pp. R9-R10, 2004.

[38] F. Haque, J. Li, H.-C. Wu, X.-J. Liang, and P. Guo, "Solid-state and biological nanopore for real-time sensing of single chemical and sequencing of dna," Nano today, vol. 8, no. 1, pp. 56-74, 2013.

[39] L. Song, M. R. Hobaugh, C. Shustak, S. Cheley, H. Bayley, and J. E. Gouaux, "Structure of staphylococcal $\alpha$-hemolysin, a heptameric transmembrane pore," Science, vol. 274, no. 5294, pp. 1859-1865, 1996.

[40] M. Van den Hout, A. R. Hall, M. Y. Wu, H. W. Zandbergen, C. Dekker, and N. H. Dekker, "Controlling nanopore size, shape and stability," Nanotechnology, vol. 21, no. 11, p. 115304, 2010. 
[41] C. A. Merchant, K. Healy, M. Wanunu, V. Ray, N. Peterman, J. Bartel, M. D. Fischbein, K. Venta, Z. Luo, A. C. Johnson, et al., "Dna translocation through graphene nanopores," Nano letters, vol. 10, no. 8, pp. 2915-2921, 2010 .

[42] M. Wanunu, S. Bhattacharya, Y. Xie, Y. Tor, A. Aksimentiev, and M. Drndic, "Nanopore analysis of individual rna/antibiotic complexes," Acs Nano, vol. 5, no. 12, pp. 9345-9353, 2011.

[43] W. Guo, H. Xia, L. Cao, F. Xia, S. Wang, G. Zhang, Y. Song, Y. Wang, L. Jiang, and D. Zhu, "Integrating ionic gate and rectifier within one solidstate nanopore via modification with dual-responsive copolymer brushes," Advanced Functional Materials, vol. 20, no. 20, pp. 3561-3567, 2010.

[44] A. R. Hall, A. Scott, D. Rotem, K. K. Mehta, H. Bayley, and C. Dekker, "Hybrid pore formation by directed insertion of $\alpha$-haemolysin into solid-state nanopores," Nature nanotechnology, vol. 5, no. 12, p. 874, 2010.

[45] N. Kasties, E. Jandciu, A. Jones, M. Wink, and R. FitzRoy, An introduction to molecular biotechnology. John Wiley \& Sons, 2006.

[46] K. Doyle, "The source of discovery: protocols and applications guide," Madison: PROMEGA, 1996.

[47] L. Buckingham, Molecular diagnostics: fundamentals, methods and clinical applications. FA Davis, 2019.

[48] G. Brooks, Biotechnology in Healthcare: An Introduction to Biopharmaceuticals. The Pharmaceutical Press, 1998.

[49] K. Kojima and S. Ozawa, "Method for isolating and purifying nucleic acids," June 14 2005. US Patent 6,905,825. 
[50] K.-H. Esser, W. H. Marx, and T. Lisowsky, "Nucleic acid-free matrix: regeneration of dna binding columns," BioTechniques, vol. 39, no. 2, pp. 270-271, 2005.

[51] D. T. Gjerde, L. Hoang, and D. Hornby, "Rna extraction and analysis," RNA Purification and Analysis, 2009.

[52] S. Berensmeier, "Magnetic particles for the separation and purification of nucleic acids," Applied microbiology and biotechnology, vol. 73, no. 3, pp. 495$504,2006$.

[53] R. D. Nargessi, "Magnetic isolation and purification of nucleic acids," Feb. 15 2005. US Patent 6,855,499.

[54] B.-N. Oy, Quickpick ${ }^{T M}$ Plasmid DNA. 2003. Turku, Finland.

[55] P. Corporation, Personal Automation ${ }^{T M}$ for Workflow Optimization in the Clinical Lab. 2008. Promega, San Luis Obispo, California, USA.

[56] A. Analytik Jena, InnuPure C12 Extraction System. 2007. Analytik Jena AG, Jena, Germany.

[57] J. Boyd, "Robotic laboratory automation," Science, vol. 295, no. 5554, pp. 517-518, 2002.

[58] J. Loeffler, K. D. Schmidt, H. Hebart, and H. Einsele, "Automated nucleic acid extraction," in Encyclopedia of Medical Genomics and Proteomics, 2 Volume Set (Print), pp. 93-96, CRC Press, 2004.

[59] S. Haeberle, S. Pausch, R. Burger, S. Lutz, F. von Stetten, R. Zengerle, and J. Ducrée, "Automation of nucleic acid extraction by a coriolis-force actuated droplet router," in Proc. of the 11th International Conference on Miniaturized Systems for Chemistry and Life Sciences, pp. 7-11, 2007.

[60] A. Mojarro, J. Hachey, J. Tani, A. Smith, S. Bhattaru, A. Pontefract, R. Doebler, M. Brown, and G. Ruvkun, "Setg: nucleic acid extraction and 
sequencing for in situ life detection on mars," in 3rd International Workshop on Instrumentation for Planetary Mission, vol. 1980, 2016.

[61] P. J. Kenis, R. F. Ismagilov, and G. M. Whitesides, "Microfabrication inside capillaries using multiphase laminar flow patterning," Science, vol. 285, no. 5424, pp. 83-85, 1999.

[62] A. Manz, N. Graber, and H. á. Widmer, "Miniaturized total chemical analysis systems: a novel concept for chemical sensing," Sensors and actuators B: Chemical, vol. 1, no. 1-6, pp. 244-248, 1990.

[63] D. J. Harrison, K. Fluri, K. Seiler, Z. Fan, C. S. Effenhauser, and A. Manz, "Micromachining a miniaturized capillary electrophoresis-based chemical analysis system on a chip," Science, vol. 261, no. 5123, pp. 895-897, 1993.

[64] N. A. Cellar, S. T. Burns, J.-C. Meiners, H. Chen, and R. T. Kennedy, "Microfluidic chip for low-flow push-pull perfusion sampling in vivo with online analysis of amino acids," Analytical chemistry, vol. 77, no. 21, pp. 70677073, 2005.

[65] C. T. Culbertson, S. C. Jacobson, and J. M. Ramsey, "Microchip devices for high-efficiency separations," Analytical Chemistry, vol. 72, no. 23, pp. 5814$5819,2000$.

[66] N. Gottschlich, C. T. Culbertson, T. E. McKnight, S. C. Jacobson, and J. M. Ramsey, "Integrated microchip-device for the digestion, separation and postcolumn labeling of proteins and peptides," Journal of Chromatography B: Biomedical Sciences and Applications, vol. 745, no. 1, pp. 243-249, 2000.

[67] S. C. Jacobson, L. B. Koutny, R. Hergenroeder, A. W. Moore, and J. M. Ramsey, "Microchip capillary electrophoresis with an integrated postcolumn reactor," Analytical Chemistry, vol. 66, no. 20, pp. 3472-3476, 1994. 
[68] S. K. Sia and G. M. Whitesides, "Microfluidic devices fabricated in poly (dimethylsiloxane) for biological studies," Electrophoresis, vol. 24, no. 21, pp. 3563-3576, 2003.

[69] N. A. Cellar and R. T. Kennedy, "A capillary-pdms hybrid chip for separations-based sensing of neurotransmitters in vivo," Lab on a Chip, vol. 6, no. 9, pp. 1205-1212, 2006.

[70] C. A. Emrich, H. Tian, I. L. Medintz, and R. A. Mathies, "Microfabricated 384-lane capillary array electrophoresis bioanalyzer for ultrahigh-throughput genetic analysis," Analytical Chemistry, vol. 74, no. 19, pp. 5076-5083, 2002.

[71] J. F. Dishinger and R. T. Kennedy, "Serial immunoassays in parallel on a microfluidic chip for monitoring hormone secretion from living cells," Analytical chemistry, vol. 79, no. 3, pp. 947-954, 2007.

[72] M. A. McClain, C. T. Culbertson, S. C. Jacobson, N. L. Allbritton, C. E. Sims, and J. M. Ramsey, "Microfluidic devices for the high-throughput chemical analysis of cells," Analytical chemistry, vol. 75, no. 21, pp. 5646$5655,2003$.

[73] J. C. McDonald and G. M. Whitesides, "Poly (dimethylsiloxane) as a material for fabricating microfluidic devices," Accounts of chemical research, vol. 35, no. 7, pp. 491-499, 2002.

[74] G. T. Roman and R. T. Kennedy, "Fully integrated microfluidic separations systems for biochemical analysis," Journal of Chromatography A, vol. 1168, no. 1-2, pp. 170-188, 2007.

[75] K. Sharp, R. Adrian, J. Santiago, and J. Molho, "The mems handbook. chapter 6. liquid flows in microchannels/eds. by m. gadel-hak," 2002.

[76] A. M. Skelley, J. R. Scherer, A. D. Aubrey, W. H. Grover, R. H. Ivester, P. Ehrenfreund, F. J. Grunthaner, J. L. Bada, and R. A. Mathies, "Development and evaluation of a microdevice for amino acid biomarker detection 
and analysis on mars," Proceedings of the National Academy of Sciences, vol. 102, no. 4, pp. 1041-1046, 2005.

[77] C. T. Culbertson, Y. Tugnawat, A. R. Meyer, G. T. Roman, J. M. Ramsey, and S. R. Gonda, "Microchip separations in reduced-gravity and hypergravity environments," Analytical chemistry, vol. 77, no. 24, pp. 7933-7940, 2005.

[78] C. J. Easley, J. M. Karlinsey, J. M. Bienvenue, L. A. Legendre, M. G. Roper, S. H. Feldman, M. A. Hughes, E. L. Hewlett, T. J. Merkel, J. P. Ferrance, et al., "A fully integrated microfluidic genetic analysis system with samplein-answer-out capability," Proceedings of the National Academy of Sciences, vol. 103, no. 51, pp. 19272-19277, 2006.

[79] E. Quirico, G. Montagnac, V. Lees, P. F. McMillan, C. Szopa, G. Cernogora, J.-N. Rouzaud, P. Simon, J.-M. Bernard, P. Coll, et al., "New experimental constraints on the composition and structure of tholins," Icarus, vol. 198, no. 1, pp. 218-231, 2008.

[80] R. Hodyss, Methods for the analysis of organic chemistry on Titan. PhD thesis, California Institute of Technology, 2006.

[81] P. Coll, D. Coscia, N. Smith, M.-C. Gazeau, S. Ramırez, G. Cernogora, G. Israel, and F. Raulin, "Experimental laboratory simulation of titan's atmosphere: aerosols and gas phase," Planetary and Space Science, vol. 47, no. 10-11, pp. 1331-1340, 1999.

[82] A. Bulu, "Chapter 7 two-dimensional flow of the real fluids." https://web. itu.edu.tr/ bulu/fluid_mechanics_files/lecture_notes_07.pdf.

Fluid Mechanics, Accessed: 2019-08-03.

[83] T. Thorsen, R. W. Roberts, F. H. Arnold, and S. R. Quake, "Dynamic pattern formation in a vesicle-generating microfluidic device," Physical review letters, vol. 86, no. 18, p. 4163, 2001. 
[84] P. Garstecki, M. J. Fuerstman, H. A. Stone, and G. M. Whitesides, "Formation of droplets and bubbles in a microfluidic t-junction-scaling and mechanism of break-up," Lab on a Chip, vol. 6, no. 3, pp. 437-446, 2006.

[85] A. Carlson, M. Do-Quang, and G. Amberg, "Droplet dynamics in a bifurcating channel," International Journal of Multiphase Flow, vol. 36, no. 5, pp. 397-405, 2010.

[86] D. Link, S. L. Anna, D. Weitz, and H. A. Stone, "Geometrically mediated breakup of drops in microfluidic devices," Physical review letters, vol. 92, no. 5, p. 054503, 2004.

[87] A. M. Leshansky and L. M. Pismen, "Breakup of drops in a microfluidic t junction," Physics of Fluids, vol. 21, no. 2, p. 023303, 2009.

[88] A. Biral and A. Zanella, "Introducing purely hydrodynamic networking functionalities into microfluidic systems," Nano Communication Networks, vol. 4, no. 4, pp. 205-215, 2013.

[89] T. F. Systems, "Miniature peristaltic pump - rp-tx series." https://www. takasago-fluidics.com/products/rp-tx. Accessed: 2020-04-25.

[90] T. F. Scientific, "Falcon 50ml conical centrifuge tubes." https://www.fishersci.ca/shop/products/ falcon-50ml-conical-centrifuge-tubes-2/p-193321. Accessed: 2020-04-25.

[91] J. Wang, "On-chip enzymatic assays," Electrophoresis, vol. 23, no. 5, pp. 713-718, 2002.

[92] K. Sakai-Kato, M. Kato, and T. Toyo'oka, "Creation of an on-chip enzyme reactor by encapsulating trypsin in sol- gel on a plastic microchip," Analytical chemistry, vol. 75, no. 3, pp. 388-393, 2003. 
[93] S.-S. Park, S. I. Cho, M.-S. Kim, Y.-K. Kim, and B.-G. Kim, "Integration of on-column immobilized enzyme reactor in microchip electrophoresis," Electrophoresis, vol. 24, no. 1-2, pp. 200-206, 2003.

[94] S. D. Mangru and D. J. Harrison, "Chemiluminescence detection in integrated post-separation reactors for microchip-based capillary electrophoresis and affinity electrophoresis," Electrophoresis, vol. 19, no. 13, pp. 2301-2307, 1998.

[95] G. R. Duarte, C. W. Price, B. H. Augustine, E. Carrilho, and J. P. Landers, "Dynamic solid phase dna extraction and pcr amplification in polyestertoner based microchip," Analytical chemistry, vol. 83, no. 13, pp. 5182-5189, 2011.

[96] M. Benhabib, T. N. Chiesl, A. M. Stockton, J. R. Scherer, and R. A. Mathies, "Multichannel capillary electrophoresis microdevice and instrumentation for in situ planetary analysis of organic molecules and biomarkers," Analytical chemistry, vol. 82, no. 6, pp. 2372-2379, 2010.

[97] M. F. Mora, F. Greer, A. M. Stockton, S. Bryant, and P. A. Willis, "Toward total automation of microfluidics for extraterrestial in situ analysis," Analytical chemistry, vol. 83, no. 22, pp. 8636-8641, 2011.

[98] P. A. Willis, B. D. Hunt, V. E. White, M. C. Lee, M. Ikeda, S. Bae, M. J. Pelletier, and F. J. Grunthaner, "Monolithic teflon@ membrane valves and pumps for harsh chemical and low-temperature use," Lab on a Chip, vol. 7, no. 11, pp. 1469-1474, 2007.

[99] W. H. Grover, M. G. von Muhlen, and S. R. Manalis, "Teflon films for chemically-inert microfluidic valves and pumps," Lab on a Chip, vol. 8, no. 6, pp. 913-918, 2008.

[100] P. A. Willis, F. Greer, M. C. Lee, J. A. Smith, V. E. White, F. J. Grunthaner, J. J. Sprague, and J. P. Rolland, "Monolithic photolithographically 
patterned fluorocur ${ }^{\mathrm{TM}}$ pfpe membrane valves and pumps for in situ planetary exploration," Lab on a Chip, vol. 8, no. 7, pp. 1024-1026, 2008.

[101] J. C. Lötters, W. Olthuis, P. H. Veltink, and P. Bergveld, "The mechanical properties of the rubber elastic polymer polydimethylsiloxane for sensor applications," Journal of micromechanics and microengineering, vol. 7, no. 3, p. 145, 1997.

[102] D. Microfluidics, "Manifold 7 ports $1 / 4$ "-28 peek." https://darwin-microfluidics.com/products/ manifold-7-ports-1-4-28-peek?variant=13826064875565. Accessed: 2020-04-25.

[103] S. On, "Dragon skin тм30." https://www.smooth-on.com/products/ dragon-skin-30/. Accessed: 2020-04-25.

[104] M. bio, "Superfastprep-2 $2^{T M}$, portable bead beating grinder and lysis system, 2 x 2 ml, battery operated." https://ca.mpio.com/superfastprep-2? SID=isaj98pehik2ega7hiv4920pf5. Accessed:2020-04-25.

[105] E. A. Oblath, W. H. Henley, J. P. Alarie, and J. M. Ramsey, "A microfluidic chip integrating dna extraction and real-time pcr for the detection of bacteria in saliva," Lab on a Chip, vol. 13, no. 7, pp. 1325-1332, 2013.

[106] C. Zhang and D. Xing, "Miniaturized pcr chips for nucleic acid amplification and analysis: latest advances and future trends," Nucleic acids research, vol. 35, no. 13, pp. 4223-4237, 2007.

[107] T. Gosiewski, L. Szała, A. Pietrzyk, M. Brzychczy-Włoch, P. B. Heczko, and M. Bulanda, "Comparison of methods for isolation of bacterial and fungal dna from human blood," Current microbiology, vol. 68, no. 2, pp. 149-155, 2014. 
[108] M. Mahalanabis, H. Al-Muayad, M. D. Kulinski, D. Altman, and C. M. Klapperich, "Cell lysis and dna extraction of gram-positive and gramnegative bacteria from whole blood in a disposable microfluidic chip," Lab on a Chip, vol. 9, no. 19, pp. 2811-2817, 2009.

[109] L. Van Heirstraeten, P. Spang, C. Schwind, K. S. Drese, M. Ritzi-Lehnert, B. Nieto, M. Camps, B. Landgraf, F. Guasch, A. H. Corbera, et al., "Integrated dna and rna extraction and purification on an automated microfluidic cassette from bacterial and viral pathogens causing community-acquired lower respiratory tract infections," Lab on a Chip, vol. 14, no. 9, pp. 15191526, 2014.

[110] A. F. Sauer-Budge, P. Mirer, A. Chatterjee, C. M. Klapperich, D. Chargin, and A. Sharon, "Low cost and manufacturable complete microtas for detecting bacteria," Lab on a Chip, vol. 9, no. 19, pp. 2803-2810, 2009.

[111] O. Salazar and J. A. Asenjo, "Enzymatic lysis of microbial cells," Biotechnology letters, vol. 29, no. 7, pp. 985-994, 2007.

[112] L. Nan, Z. Jiang, and X. Wei, "Emerging microfluidic devices for cell lysis: a review," Lab on a Chip, vol. 14, no. 6, pp. 1060-1073, 2014.

[113] C.-H. Wang, K.-Y. Lien, J.-J. Wu, and G.-B. Lee, "A magnetic bead-based assay for the rapid detection of methicillin-resistant staphylococcus aureus by using a microfluidic system with integrated loop-mediated isothermal amplification," Lab on a Chip, vol. 11, no. 8, pp. 1521-1531, 2011.

[114] T. Tandiono, D. S.-W. Ow, L. Driessen, C. S.-H. Chin, E. Klaseboer, A. B.H. Choo, S.-W. Ohl, and C.-D. Ohl, "Sonolysis of escherichia coli and pichia pastoris in microfluidics," Lab on a Chip, vol. 12, no. 4, pp. 780-786, 2012.

[115] A. Talebpour, R. Maaskant, A. A. Khine, and T. Alavie, "Use of surface enhanced blocking (seb) electrodes for microbial cell lysis in flow-through devices," PloS one, vol. 9, no. 7, 2014. 
[116] J.-G. Lee, K. H. Cheong, N. Huh, S. Kim, J.-W. Choi, and C. Ko, "Microchip-based one step dna extraction and real-time pcr in one chamber for rapid pathogen identification," Lab on a Chip, vol. 6, no. 7, pp. 886-895, 2006 .

[117] J. Kim, J. W. Hong, D. P. Kim, J. H. Shin, and I. Park, "Nanowireintegrated microfluidic devices for facile and reagent-free mechanical cell lysis," Lab on a chip, vol. 12, no. 16, pp. 2914-2921, 2012.

[118] M. Hohnadel, L. Felden, D. Fijuljanin, S. Jouette, and R. Chollet, "A new ultrasonic high-throughput instrument for rapid dna release from microorganisms," Journal of microbiological methods, vol. 99, pp. 71-80, 2014.

[119] A. Alexopoulos, D. Maggioris, and C. Kiparissides, "Cfd analysis of turbulence non-homogeneity in mixing vessels: A two-compartment model," Chemical Engineering Science, vol. 57, no. 10, pp. 1735-1752, 2002.

[120] V. C. Patel, W. Rodi, and G. Scheuerer, "Turbulence models for near-wall and low reynolds number flows-a review," AIAA journal, vol. 23, no. 9, pp. 1308-1319, 1985.

[121] "ANSYS ${ }^{\circledR}$ CFX, release 2019 r1,"

[122] F. R. Menter, "Two-equation eddy-viscosity turbulence models for engineering applications," AIAA journal, vol. 32, no. 8, pp. 1598-1605, 1994.

[123] A. Brucato, M. Ciofalo, F. Grisafi, and G. Micale, "Numerical prediction of flow fields in baffled stirred vessels: a comparison of alternative modelling approaches," Chemical Engineering Science, vol. 53, no. 21, pp. 3653-3684, 1998.

[124] J. C. McDonald, S. J. Metallo, and G. M. Whitesides, "Fabrication of a configurable, single-use microfluidic device," Analytical chemistry, vol. 73, no. 23, pp. 5645-5650, 2001. 
[125] J. D. Anderson and J. Wendt, Computational fluid dynamics, vol. 206. Springer, 1995.

[126] H. Patel, F. Ein-Mozaffari, and R. Dhib, "Cfd analysis of mixing in thermal polymerization of styrene," Computers $\&$ chemical engineering, vol. 34, no. 4, pp. 421-429, 2010.

[127] D. Andersen, W. Pollard, C. McKay, J. Heldmann, O. Toon, and B. Jakosky, "Cold springs in permafrost on earth and mars," in Bulletin of the American Astronomical Society, vol. 32, p. 1639, 2000.

[128] W. Pollard, C. Omelon, D. Andersen, and C. McKay, "Perennial spring occurrence in the expedition fiord area of western axel heiberg island, canadian high arctic," Canadian Journal of Earth Sciences, vol. 36, no. 1, pp. 105-120, 1999. 


\section{Appendix A}

\section{Drawing Sheets for the 3D}

\section{Models}

All the dimensions specified in the drawing sheets are in millimeter ( $\mathrm{mm}$ ). 


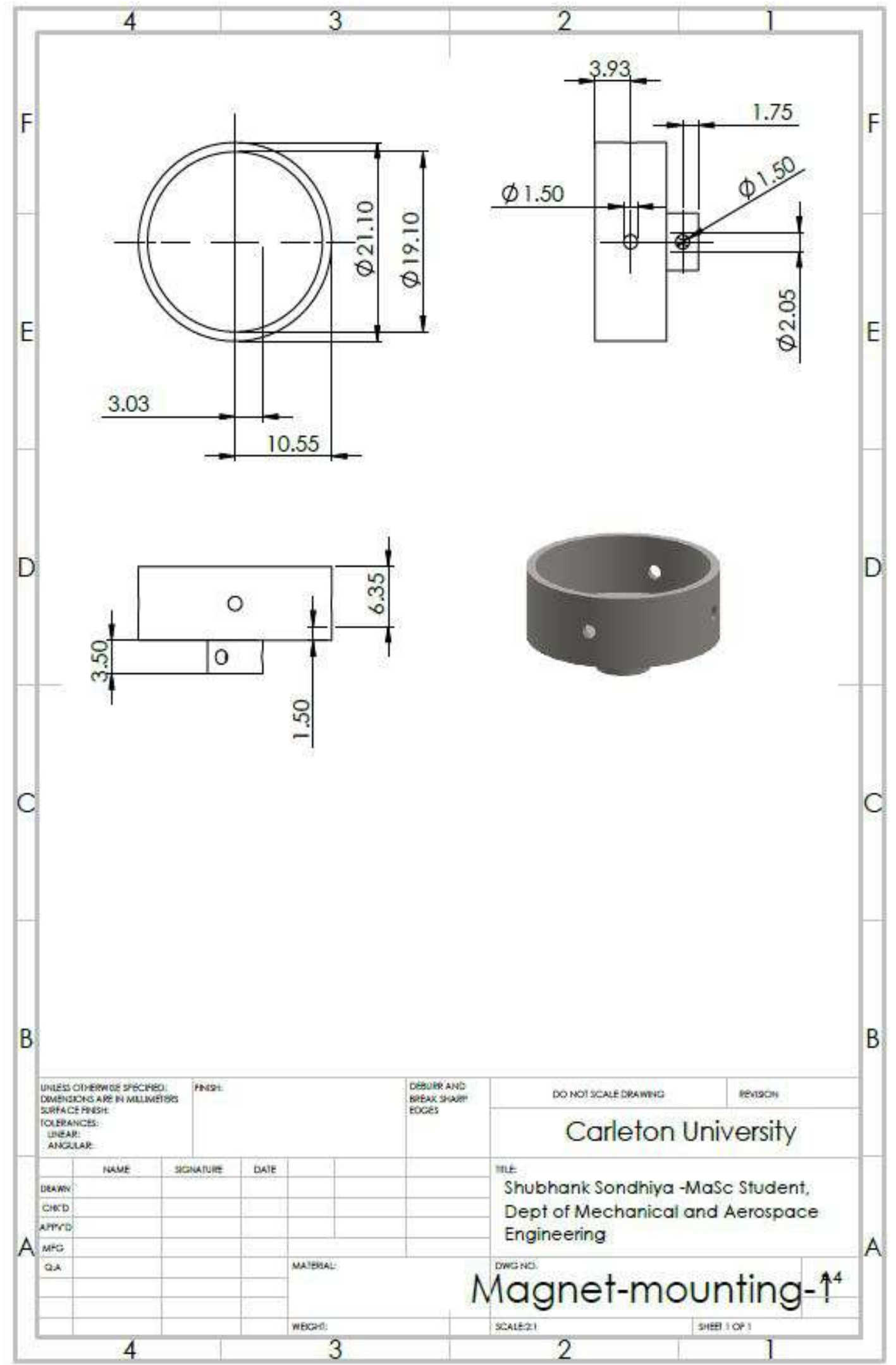




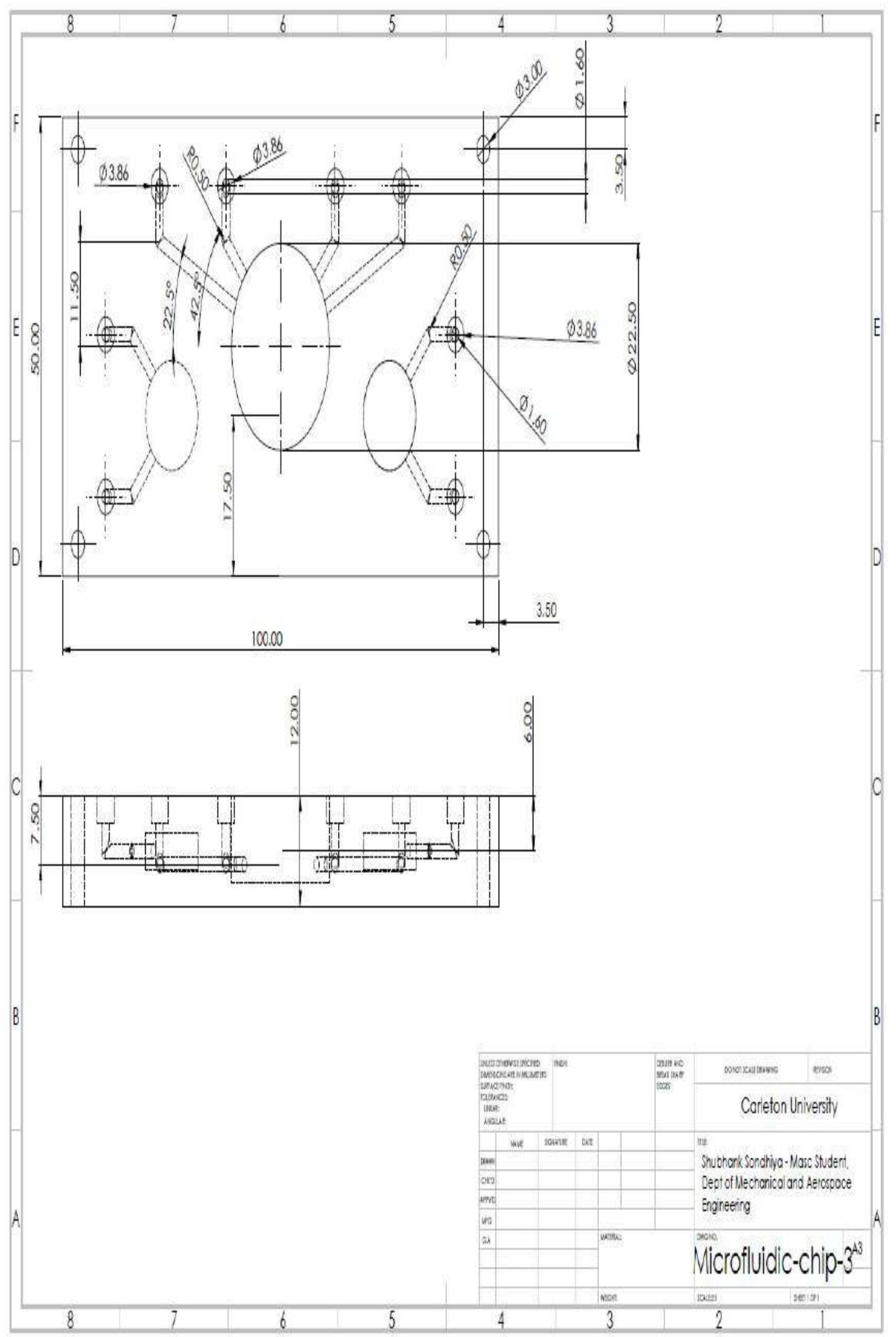




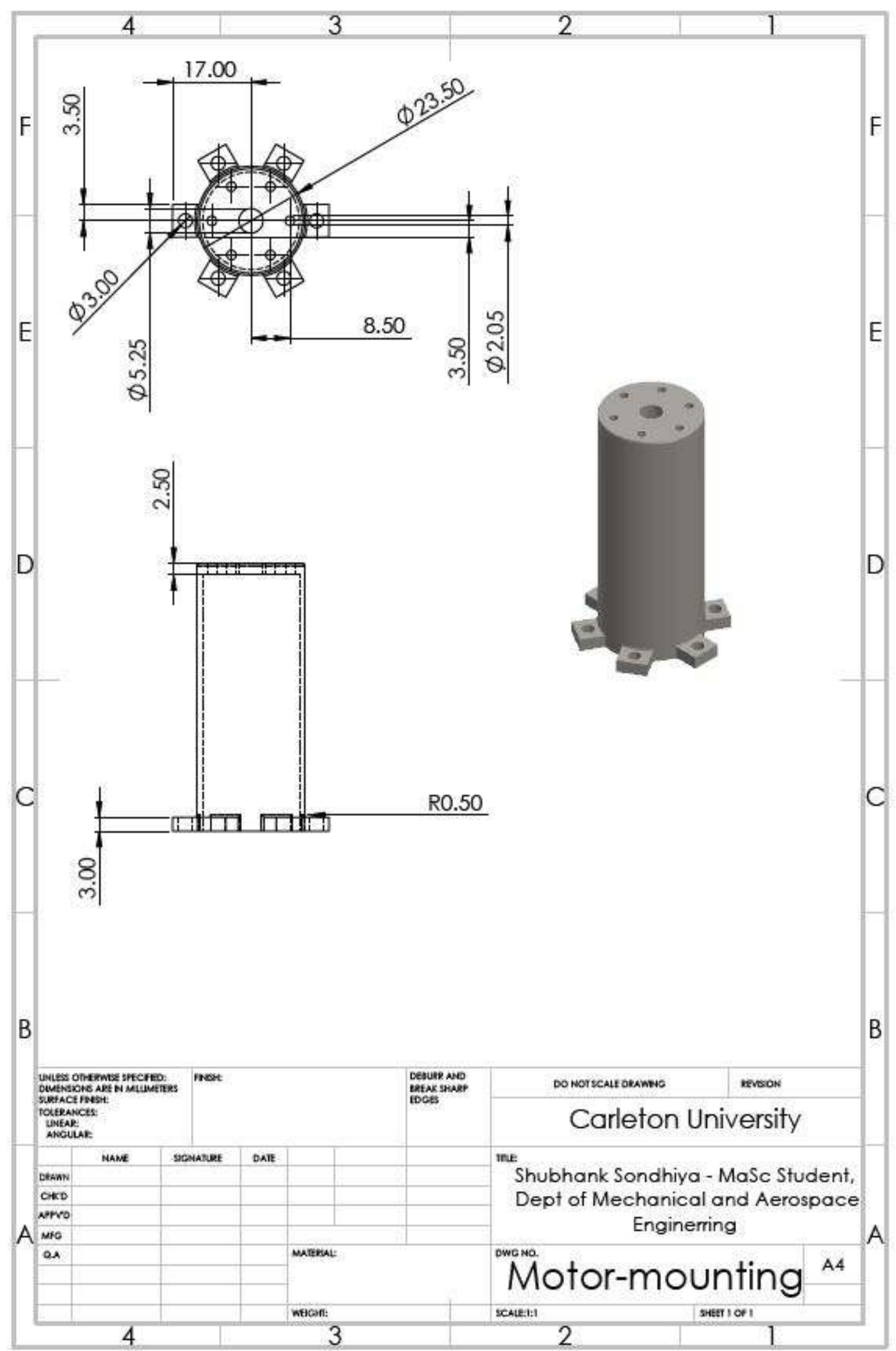




\section{Appendix B}

\section{Data and Instruction Sheets}

B.1 Data sheet for W1209 Temperature Control

Module 


\section{SPECIFICATION:}

Temperature Control Range: $-50 \sim 110 \mathrm{C}$

Resolution at -9.9 to $99.9: 0.1 \mathrm{C}$

Resolution at all other temperatures: $1 \mathrm{C}$

Measurement Accuracy: $0.1 \mathrm{C}$

Control Accuracy: $0.1 \mathrm{C}$

Refresh Rate: 0.5 Seconds

Input Power (DC): $12 \mathrm{~V}$

Measuring inputs: NTC (10K 0.5\%)

Waterproof Sensor: 0.5M

Output: 1 Channel Relay Output, Capacity: $10 \mathrm{~A}$

Power Consumption

Static Current: $<=35 \mathrm{~mA}$

Current: $<=65 \mathrm{~mA}$

Environmental Requirements

Temperature: $-10 \sim 60 \mathrm{C}$

Humidity: $20-85 \%$

Dimensions

$48 \mathrm{~mm} \times 40 \mathrm{~mm} \times 14 \mathrm{~mm}$

\section{Settings Chart}

Long press the "SET" button to activate the menu.

Code Description Range Default Value

PO Heat $\mathrm{C} / \mathrm{HC}$

P1 Backlash Set 0.1-15 2

P2 Upper Limit 110110

P3 Lower Limit - $50-50$

P4 Correction $-7.0 \sim 7.00$

P5 Delay Start Time $0-10$ mins 0

P6 High Temperature Alarm 0-110 OFF

Long pressing +- will reset all values to their default

Displaying the current temperature:

The thermostat will display the current temperature in $\mathrm{o}$ by default. When in any other mode making no input for approximately 5 seconds will cause the thermostat to return to this defauit display. 
Setting the trigger temperature:

To set the trigger temperature press the button marked "SET". The seven segment dispiay will flash. You can now set a trigger temperature (in oc) using the ' + ' and '-' buttons in 0.1 degree increments. If no buttons are pressed for approximately 2 seconds the trigger temperature will be stored and the display will return back to the current temperature.

Setting the parameters:

To set any parameter first long press the 'SET' button for at least 5 seconds. The seven segment display should now display 'PO'. This represents parameter PO. Pressing the '+' or '-' buttons will cycle though the various parameters (PO to P6). Pressing the 'SET' button whilst any of there parameters are displayed will allow you to change the value for that parameter using the ' 4 ' and '-' buttons (see below). When finished setting a parameter press the set button to exit that option. If no buttons are pressed for approximately 5 seconds the thermostat will exit the parameter options and will return back to the default temperature display.

Setting the cooling or heating parameter P0:

The parameter PO has two settings, $\mathrm{C}$ and $\mathrm{H}$. When set to $\mathrm{C}$ (default) the relay will energise when the temperature is reached. Use this setting if connecting to an air-conditioning system. When set to $H$ the relay will de-energise when the temperature is reached. Use this setting if controlling a heating device.

Setting the hysteresis parameter P1:

This sets how much change in temperature must occur before the relay will change state. For example if set to the default $20 \mathrm{C}$ and the the trigger temperature has been set to $250 \mathrm{C}$, it will not deenergise until the temperature falls back below below $230 \mathrm{C}$. Setting this hysteresis helps stop the thermostat from continually triggering when the temperature drifts around the trip temperature.

Setting the upper limit of the thermostat parameter P2:

This parameter limits the maximum trigger temperature that can be set. It can be used as a safety to stop an excessively high trigger temperature from accidentally being set by the user.

Setting the lower limit of the thermostat parameter P3:

This parameter limits the minimum trigger temperature that can be set. It can be used as a safety to stop an excessively low trigger temperature from accidentally being set by the user.

Setting temperature offset correction parameter P4:

Should you find there is a difference between the displayed temperature and the actual temperature (for instance if the temperature probe is on a long run of cable) you can make minor corrections to the temperature reading with this parameter.

Setting the trigger delay parameter P5:

This parameter allows for delaying switching of the relay when the trigger temperature has be reached. The parameter can be set in one minute increments up to a maximum of 10 minutes.

Setting the high temperature alarm parameter P6:

Setting a value for this parameter will cause the relay to switch off when the the temperature reaches this setting. The seven segment display will also show '-' to indicate an alarm condition. The relay will not re-energise until the temperature falls below this value. The default setting is OFF. 


\section{B.2 Instructions Sheet for Dragon Skin ${ }^{\mathrm{TM}}$ Series}

\section{Dragon Skin ${ }^{\mathrm{Tm}}$ Series Addition Cure Silicone Rubber Compounds \\ PRODUCT OVERVIEW}

Dragon Skin"s silicones are high performance platinum cure liquid slikcone compounds that are used for a varlety of applications ranging from creating skin effects and other movie special effects to making production molds for casting a variety of materials. Because of the superior physical properties and flexibility of Dragon Skin" rubbers, they are also used for medical prosthetics and cushioning applications. Dragon Skin" rubbers are also used for a variety of industrial applications and have a service temperature range of a constant $-65^{\circ} \mathrm{F}$ to $+450^{\circ} \mathrm{F}\left(-53^{\circ} \mathrm{C}\right.$ to $\left.+232^{\circ} \mathrm{C}\right)$.

Great for Making Moids for a Variety of Applications - Avaliable in Shore 10A, 20A and 30A, Dragon Skin" silicones can be used to make exceptionally strong and tear resistant molds for casting plaster, wax, concrete (timited production run), resins and other materials. Dragon Skin" $10 \mathrm{AF}$ is an anti-fungal silicone sultable for making a variety of skin-safe cushioning device configurations that resist fungi for orthopedic and orthotic applications.

Time Tested, Versatile Special Effects Moterial - Soft, super-strong and stretchy, Dragon Skin ${ }^{21} 10$ (Very Fast, Fast, Medium and Slow speeds) is used around the world to make spectacular skin and creature effects. An infinite number of color effects can be achieved by adding Silc Pig "silicone pigments or Cast Magic" effects powders. Cured rubber can also be painted with the Psycho Paint" system. Cured material is skin safe and certified by an independent laboratory.

Easy To Use - Dragon Skinn sillcones are mixed 1A:1B by weight or volume. Liquid rubber can be thinned with Silicone Thinner or thickened with $T H H \cdot V E X^{i v}$. Aubber cures at room temperature $\left.173^{\circ} / 23^{\circ} \mathrm{C}\right)$ withnegligible shrinkage. Vacuum degassing is recommended to minimize air bubbles in cured rubber.

\begin{tabular}{|c|c|c|c|c|c|c|c|c|c|c|c|}
\hline \multicolumn{12}{|c|}{ TECHNICAL OVERVIEW } \\
\hline & 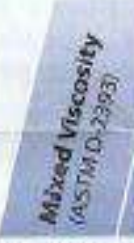 & 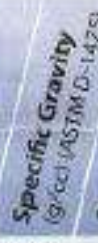 & 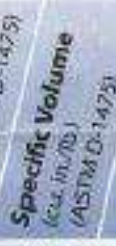 & (5) & है & 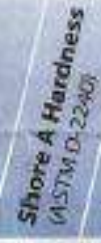 & 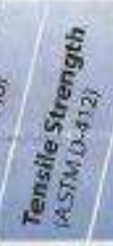 & 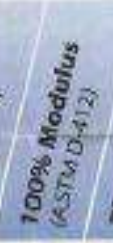 & 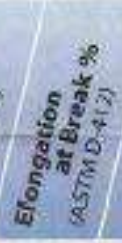 & 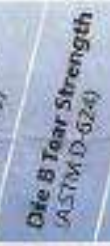 & $\begin{array}{l}\text { है } \\
5 \% \\
50 \\
5 \% \\
\text { है है } \\
\text { कह के }\end{array}$ \\
\hline Fast & $m a x$ & & 258 & & amin. & ex & & 22055 & 10005 & $102 \mathrm{pl}$ & ont indin. \\
\hline & & 100 & 258 & & $75 \mathrm{~min}$. & & & " & $1000 \%$ & $102 \rho^{6}$ & $1 \operatorname{lin} / \mathrm{in}$. \\
\hline edium & & 1.07 & 25 & $20 \mathrm{~min}$. & Shours & $10 A$ & 75 pst & 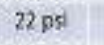 & 700056 & 102 phi & 001 in in. \\
\hline & & 10 & & & Thours & $10 A$ & & & $1000 \%$ & $102 \mathrm{pli}$ & $.001 \mathrm{in} / \mathrm{in}$ \\
\hline & & 10 & 258 & & 5 hours & $10 A$ & $475 \mathrm{psi}$ & $p s i$ & $1000 \%$ & $102 \mathrm{pH}$ & 5.001 in ${ }^{2}$ in. \\
\hline & & 1.08 & & & 4 hours & $20 \mathrm{~A}$ & & $49 \mathrm{psi}$ & $620 \%$ & $120 \mathrm{pll}$ & c.opi hyin. \\
\hline tgon Skist - 30 & & .06 & 5.7 & $45 \mathrm{~min}$. & 16 hours & $33 A$ & $500,05 i$ & $86 \mathrm{ps}$ & $36 \% \%$ & $108 \mathrm{pli}$ & $<.00 \mathrm{in} / \mathrm{h}$ \\
\hline
\end{tabular}

Mix Ratio: 1 A:18 by volume or weight

Useful Temperature Range: $65^{\circ} \mathrm{F}$ to $+450^{\circ} \mathrm{F}\left(-53^{\circ} \mathrm{C}\right.$ to $\left.+232 \mathrm{C}\right)$ Color:Translucent Dielectric Strength (ASTM D-149): $>350$ woltsimil

\section{PROCESSING RECOMMENDATIONS}

-All values medsered after 7 days at $73^{\circ} F / 23^{\circ} \mathrm{C}$

PREPARATION... Safety - Use in a properly ventilated afea ("room size' ventilation). Wear safety glasses, long sieeves and rubber gloves to minimize contamination risk. Wear vinyl gloves only. Latex gloves will inhibit the cure of the rubber.

Store and use material at room temperature $\left(73^{\circ} \mathrm{F} / 23^{\circ} \mathrm{C}\right.$ ). Warmer temperatures will drastically reduce working time and cure time. Storing material at warmer temperatures will also reduce the usable sheif life of unused material. These products have a limited sheif life and should be used as soon as possible. Mixing containers should have straight sides and a flat bottom. Mlixing sticks should be flat and stiff with defined edges for scraping the sides and bottom of your maxing container.

Cure Imhibition - Addition-cure silicone rubber may be inhibited by certain contaminants in or on the pattern to be molded resulting in tackiness at the pattern interface or a total lack of cure throughout the mold. Latex, tin-cure silicone, sulfur clays, certain wood surfaces, newly cast polyester, epoxy, tin cure silicone rubber or urethane rubber may cause inhibition. If compat bility between the rubber and the surface is a concern, a small-scale test is recommended. Apply a small amount of rubber onto a non-critical area of the pattern. Inhibition has occurted if the rubber is gummy or uncured after the recommended cure time has passed.

Because no two applications are quite the same, a small test application to determine suitability for your project is recommended if performance of this material is in question. 


\section{Safety First:}

The Material Safety Data Sheet (MSDS) for this or any Smooth-On product should be read prior to use and is available upon request from Smooth-On. All Smcoth-On products are safe to use if directions are read and followed carefully.

\section{Keep Out of Reach of Children}

Be careful. Use only with adequate ventilation. Contact with skin and eyes may cause irritation. Flush eyes with water for 15 minutes and seek immediate medical attention. Remove from skin with waterless hand cleaner followed by soap and water.

Important: The information contained in this bulletin is considered accurate However, no warranty is expressed or impled regarding the accuracy of the data. the results to be obtained from the use thereot, or that any such use will not infringe upon a patent. User shall determine the suitability of the product for the intended application and assume all risk and liability whatsoever in connection therewith.

Cure Inhibition - To prevent inhibition, one or more coatings of a clear acrylic lacquer applied to the model surface is usually effective. Allow any sealer to thoroughly dry before applying rubber, Note: Even with a sealer, platinum silicones will not work with modeling clays containing heavy amounts of sulfur. Do a small scale test for compatibility before using on your project.

Applying A Release Agent - Aithough not usually necessary, a release agent will make demolding easier when pouring into or over most surfaces, Ease Releasen 200 is a proven release agent for making molds with silicone rubber. Mann Ease Releasev products are available from Smooth-On or your Smooth On distributor.

IMPORTANT: To ensure thorough coverage, lightly brush the release agent with a soft brush over all surfaces of the model. Follow with a light mist coating and let the release agent dry for 30 minutes.

If there is any question about the effectiveness of a sealer/release agent combination. a small-scale test should be made on an identical surface for trial.

\section{MEASURING \& MIXING...}

Stir Part A and Part B thoroughly before dispensing. After dispensing required amounts of Parts $A$ and 8 into mixing container ( 1 A:18 by volume or weight), mix thoroughly for 3 minutes making sure that you scrape the sides and bottom of the mixing container several times. After mixing parts $A$ and $B$, vacuum degassing is recommended to eliminate any entrapped air. Vacuum material for 2-3 minutes (29) inches of mercuryl, making sure that you leave encugh room in container for product volume expansion.

\section{POURING, CURING \& MOLD PERFORMANCE...}

For best results, pour your mixture in a single spot at the lowest point of the containment field Let the rubber seek its level up and over the model. $A$ unilorm flow will help minimize entrapped air. The liquid rubber should level off at least $1 / 2$ $(1.3 \mathrm{~cm})$ over the highest point of the model surface.

Curing / Post Curing - Allow rubber to cure as prescribed at room temperature $\left(73^{\circ} \mathrm{F} / 23^{\circ} \mathrm{C}\right)$ before demolding. Do not cure rubber where temperature is less than $65^{\circ} \mathrm{F} / 18^{\circ} \mathrm{C}$. Optional: Post curing the mold will aid in quickly attaining maximum physical and performance properties. After curing at room temperature, expose the rubber to $176^{\circ} \mathrm{F} / 90^{\circ} \mathrm{C}$ for 2 hours and $212 \% / 100^{\circ} \mathrm{C}$ for one hour. Allow mold to cool to room temperature before using.

If Using As A Mold - When first cast, silicone rubber molds exhibit natural release characteristics. Depending on what is being cast into the mold, mold lubricity may be depleted over time and parts will begin to stick. No release agent is necessary when casting wax or gypsum. Applying a release agent such as Ease felease" 200 (avallable from Smooth-On) prior to casting polyurethane, polyester and epoxy resins is recommended to prevent mold degradation.

Thickening Dragon Skin "h Silicones - THI-VEX" is made especially for thickening Smooth-On's silicones for vertical surface application (making brush-on molds). Different viscosities can be attained by varying the amount of THI-VEX". See the THI-VEX"s technical bulletin (available from Smooth-On or your Smooth-On distributor) for full details.

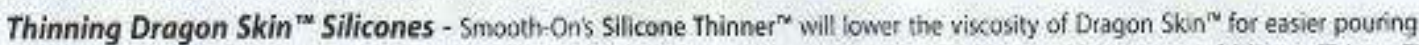
and vacuum degassing. A disadvantage is that ultimate tear and tensile are reduced in proportion to the amount of Silicone Thinner" added. It is not recommended to exceed $10 \%$ by weight of total system $(A+B)$. See the Silicone Thinner ${ }^{\text {int }}$ technical bulletin [available from Smooth-On or your Smooth-On distributorl for full details.

Mold Performance \& Storage - The physical life of the mold depends an how you use it (materials cast, frequency, etc). Casting abrasive materials such as concrete can quickly erode mold detak, while casting non-abrasive materials (wax) will not affect moid detail. Before storing, the mold should be cleaned with a soap solution and wiped fully dry. Two part (or more) molds should be assembled. Molds should be stored on a level surface in a cool, dry environment.

\section{SMOOTHEDN \\ Call Us Anytime With Questions About Your Application. Toll-free: (800) 381-1733 Fax: (610) 252-6200}

The new www.smooth-on.com is loaded with information about mold making, casting and more. 
Appendix C

IAC Conference Paper 
$70^{\mathrm{L}}$ International Astronautical Congress (IAC), Washington D. C., United States, $21-25$ October 2019.

Copyright $c 2019$ by the Intemational Astronautical Federation (IAF). All rights reserved.

\section{IAC-19,A1,6,9x50396}

\section{Direct Astrobiological Sampling of Enceladus' Subsurface Vents for the} Micro Life Instrument Suite

Shubhank Sondhiyaa ${ }^{a}$, Dr Alex Ellery ${ }^{a^{n}}$

"Space Exploration and Engineering Group, Carleton University, Canada, shubhank.sondhiya a carleton.ca, acllery a mae.carleton.ca:

"Corresponding author

\section{Abstract}

The icy moon of the solar system signifies the most auspicious targets for astrobiological exploration. Direct access to the geyser vents of Enceladus would be highly desirable to acquire pristine biological samples. Over the past few decades irrespective of multiple orbital and lander mission still relatively not much is known about the microbial presence and the biological composition. We have investigated the possibility of deploying penetrators to Enceladus to target the geyser vents to gain access to pristine or near-pristine subsurface samples. Penetrators are small missile type entry descent and landing vehicles that can carry modest scientific instruments capable of withstanding impact into the subsurface. The instrumentation used for anv biochemical analysis on astrobiological bodies such as Enceladus should perform experiments with extreme precision and sensitivity such that it does not contaminate the sample and at the same time depict the results with high throughput. We have examined a subset of instruments that might be deployed on a penetrator based on current work in developing an astrobiological suite of a robotic instrument for Mars Rover deployment comprising a micro-assay and a nanopore instrument that works on extreme environments. For the microfluidics, we have explored to minimize the sample pre-processing required for the instruments and tested the microfluidic system which could perform experimentations that might detect life from the environmental sample collected from the Mars analogue sites. Here we describe a fully automated microfluidic device capable of performing end-to-end analyses of DNA/RNA preparation and sequencing by using microbial activity micro-assay ( $\mu$-MAMA) and oxford nanopore instruments. The system integrates an array of solenoid valves, peristaltic pumps and membrane filters for autonomous fluidic routing through the channels. The use of such an integrated device dramatically reduces the analysis time, reagent consumption, costs and overall mass of the subsystem. This miniaturized and sophisticated astrobiological instrument suite offers high potential for astrobiological prospecting in a highly challenging environment including Europa.

Keywords: Astrobiological exploration, Enceladus, Miniaturized automated microfluidic system, pristine samples.

\section{Introduction}

In order to comprehend the potential for past, present and future life in extraterrestrial bodies, it becomes evident to perform DNA-related tests as it is one essential component of life that stores and transmits genetic information. The DNA is recognised as the blueprint or foundation that contains the information needed for encrypting the proteins and other cell components Nucleic Acids are considered as unambiguous signs of extant life and are an excellent candidate for biosignature detection. These nucleic acids can be detected using nanopore sequencing technologies. Nanopore based DNA sequencing technology has become one of the mos captivating and engaging third-generation technologies because of its outstanding characteristics of label-free amplification free, great read length, high throughpu which makes it plausible for high-quality gene sequencing applications. The nanopore-based DNA sequencing was first proposed by Church et al. in 1995 [1]. One year later, Kasianowicz et al. [2] demonstrated the detection of the electronic behaviour of the single- stranded DNA (ssDNA) passing through an $\alpha$-hemolysin $(2.6 \mathrm{~nm}$ in diameter) nanopore, which marked the beginning of the nanopore-based DNA sequencing field The biological nanopores have many advantages $[3,4]$. such as the dimensional reproducibility, the compatibility of the genetic or chemical modification process, and relatively slower DNA translocation velocity through the nanopores. However, there are some undeniable drawbacks $[3][4]$ such as the rigour environmental demands (i.e. temperature electrolyte concentration and $\mathrm{PH})$ of the nanopore to keep their biological activities.

With the rapid development of advanced fabrication technologies, solid-state nanopores have become an inexpensive and superior alternative to biological nanopores due to following superiorities [5][6][7][8] 1. Better robustness and durability, 2. Superior mechanical, chemical and thermal characteristics and 3 . The easier handled shape and the size fabrication process with nanometre precision. The components of an ideal commercial sequencing system using electrical 
measurements would consist of a disposable detector chip containing an array of nanopores having the required integrated microfluidic and electronic probes, a portable system that would control the fluids and the electronic elements of the chip and processes the raw sequence data Assuming one chip would be required to sequence a sample with the complexity of the genome from the analogue sites, the cost of the complete genomic sequencing will comprise the cost of preparing the genomic DNA from a biological sample, the cost of the nanopore instrument and the cost of the disposable detector chip

A very interesting research challenge consists in developing basic microfluidic systems that can interconnect specialized microfluidic devices by mean of a flexible and modular microfluidic network, thus dramatically enlarging the capabilities of microfluidic systems. A possible solution consists in electrohydrodynamic actuation [10][11], however, this would require specific and complex circuitry with many electrical connections and a high-power source which would not be a feasible solution for space exploration missions. For some of these applications, the microfluidic circuit is designed in such a way that a reactant of some kind, named dispersed phase, is inserted in the form of droplets into another immiscible fluid called the continuous phase, that acts as the carrier and conveys the reactant to microfluidic system where the reactions take place.

Most of these problems are solved by adopting purely hydrodynamic approach that only relies on the channel geometries, and on the actuators at the periphen of the system that can be controlled in order to regulate the hydrodynamic forces that act on the fluids, thus driving the droplet in the network. The basic functional principle of purely hydrodynamic control of the microfluidic systems is that droplets flow along the path of minimum instantaneous hydraulic resistance, meanwhile increasing the resistance of the channel they are crossing. Thus, an isolated droplet entering a $\mathrm{T}$ or $\mathrm{Y}$ junction through the inlet will proceed towards the outle with minimum instantaneous hydraulic resistance, while a closely following droplet may be driven to the other outlet [9][12]. Furthermore, the time behaviour of a droplet is also difficult to predict, because the motion of the droplets across the channels will continuously change the hydraulic resistance of different parts of the network and, hence the flow rates of the continuous phase in the different channel that, in turn, affect the speed of the droplets [13]. However, the behaviour in complex microfluidic systems is affected by severa interdependent factors that make the fluid flow at any one location depending upon the properties of the entire system.
A significant aim of this research paper is to bring complex laboratory workflows into small and portable devices that can be independently used via computer control. Because of the small size of the sample used (microlitres or less), low volumes are manipulated resulting in reduced reagent consumption and facilitated analysis of microscale sample. Besides the distinct approach to the problem, an integrated device should substantially reduce the analysis time, reagen consumption, costs and overall mass of the subsystem. To develop a better understanding of the problem, we would define the necessary mathematical models of the main microfluidic elements that capture the fluid flow across the channels. These models will serve as a platform in the selection of the appropriate instruments and to integrate multiple analytical and sample processing functions, such as sampling, filtering, dilution, mixing and separation onto a microfluidic assay and nanopore instruments. To validate the model, we implemented a series of experiments in the laboratory, by using a representative microfluidic system, consisting of peristaltic pumps, tubes, solenoid valves, temperaturecontrolled chamber and microbial activity micro-assay ( $\mu$-MAMA). Finally, this paper provides the following novel contributions:

1. A mathematical model to predict the flow across the channels to predict the behaviour of the fluid in the microfluidic circuits.

2. A fully automated prototype for end-to-end analyses of DNA/RNA preparation and sequencing by using novel $\mu$-MAMA design and oxford nanopore instruments in the circuitry.

The remaining of the paper is organised as follows. A brief introduction to the fundamental notion of microfluidics, along with the main notion used in this paper is discussed in section 2. The equations obtained would then be used to discuss the methodology implemented for automating the device (section 3). Section 4 discusses the experimental validation of the model. In addition, this section also focuses on the instruments used to successfully automate the process and the steps taken for miniaturization of the whole circuitry so that it could be deployed on a penetrator. Section 5 summarises and concludes the work with some considerations and discusses the potential for the successful deployment for astrobiological prospecting in a highly challenging environment such as Europa, Enceladus and Mars.

\section{Mathematical Model for microfluidic system}

To understand the fluid flow across the circular cross-section, we would assume that the fluid is incompressible and Newtonian, the flow is laminar, and 
the circular cross-section of the tube is substantially longer than its diameter. Also, there is no relative motion between the fluid particles and the solid boundaries, that is, no-slip of fluid particles at the solid boundary.

2.1 Contimıous phase

In a droplet-based microfluidic network, a fluid names continuous phase, with a dynamic viscosity $\mu_{c}[\mathrm{~Pa}$ s] is injected into the channels by an external source, in this case, a peristaltic pump that would impose a pressure

Table 1: General terminology used in this paper with the unit and its meaning.

\begin{tabular}{|c|c|c|}
\hline Symbol & Meaning & Unit \\
\hline $\mathrm{U}_{\max }$ & Maximum Velocity & {$[\mathrm{m} / \mathrm{s}]$} \\
\hline $\mathrm{V}$ & Average velocity & {$[\mathrm{m} / \mathrm{s}]$} \\
\hline$\mu_{\mathrm{c}}$ & $\begin{array}{l}\text { Continuous phase } \\
\text { viscosity }\end{array}$ & [mPa-s] \\
\hline$\mu_{\mathrm{d}}$ & $\begin{array}{l}\text { Dispersed phase } \\
\text { viscosity }\end{array}$ & [mPa-s] \\
\hline $\mathrm{r}_{0}$ & $\begin{array}{l}\text { Radius of the } \\
\text { circular cross- } \\
\text { section }\end{array}$ & {$[\mathrm{m}]$} \\
\hline$\Delta P$ & Pressure drop & {$[\mathrm{Pa}]$} \\
\hline $\mathrm{l}_{\mathrm{d}}$ & Droplet length & [m] \\
\hline $\mathrm{L}$ & Channel length & [m] \\
\hline $\mathrm{R}(\mu, \mathrm{L})$ & $\begin{array}{l}\text { Microfluidic } \\
\text { resistance }\end{array}$ & [m] \\
\hline$\delta$ & $\begin{array}{l}\text { Inter-droplet } \\
\text { distance }\end{array}$ & {$[\mathrm{m}]$} \\
\hline
\end{tabular}

gradient $\Delta P[\mathrm{~Pa}]$ across the channel. The average velocity $\mathrm{V}$, of the fluid through the channel is $\frac{Q_{c}}{A}$, or

$$
V=\frac{Q_{c}}{\pi r_{0}^{2}}=\frac{1}{8 \mu_{c}}\left(-\frac{\partial p}{\partial x}\right) r_{0}^{2}
$$

where, $r_{o}$ is the radius of the circular cross-section and $Q_{c}$ is the volumetric flow rate given by,

$$
Q_{c}=\frac{\pi}{8 \mu_{c}}\left(-\frac{\partial p}{\partial x}\right) r_{0}^{2}
$$

The pressure gradient $\frac{\partial p}{\partial x}$ in the direction of the flow depend on the $x$ only for a given case of laminar flow. A negative sign indicates a decrease of the fluid pressure in the direction of flow in a horizontal tube since flow work must be performed to compensate for the frictional resistance to the flow. Now the pressure drop between $p_{1}$ and $p_{2}$ for any stretch of the tube between $x_{1}$ and $x_{2}$. $L=x_{2}-x_{1}$ is given by,

$$
-\int_{p_{1}}^{p_{2}} \partial p=\frac{8 \mu V}{r_{0}^{2}} \int_{x_{1}}^{x_{2}} \partial x
$$

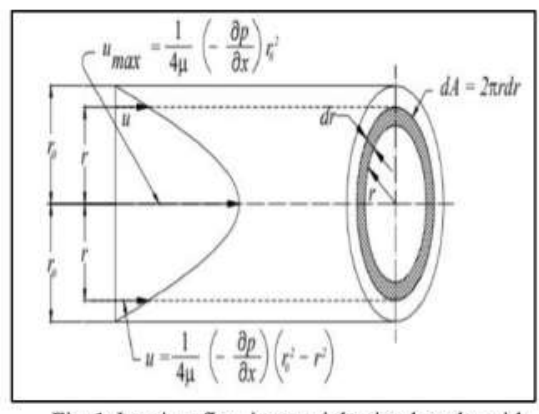

Fig. 1: Laminar flow in a straight circular tube with radius $r_{o}[14]$

and since diameter $\mathrm{D}$ equals $2 r_{0}$.

$$
\Delta P=\frac{8 \mu_{c} V L}{r_{0}{ }^{2}}=\frac{32 \mu_{c} V L}{D^{2}}
$$

where pressure drop $\Delta P=p_{1}-p_{2}$. The above equation is usually referred to as the Hagen-Poiseuille equation. To calculate the average volumetric flow rate $Q_{c}$, in the microfluidic channel of length $\mathrm{L}$ is given by the law,

$$
R_{h}=\frac{\Delta P}{Q_{c}}
$$

Here, $R_{h}$ is the microfluidic flow resistance that quantifies an energy drop along the microfluidic channels. Just like the electrical potential drop $d V$ is proportional to intensity $A$, fluid pressure drop $d P$ is proportional to the mean flow rate $Q_{c}$ and resistance $R_{h}$ can be defined as the proportional coefficient. Thus, the microfluidic resistance $R_{h}$, across a simple circular crosssection is given by the following equation,

$$
R_{h}=\frac{128 \mu_{c} L}{\pi D^{4}}
$$

From the above equation, we can say that factors that must affect microfluidic resistance depend on the following:

1. Cross-section area and length of the tube.

2. Type of fluid flowing and

3. Inside finishing of the tube.

Also, longer channels with smaller cross-section area would have higher microfluidic resistance and vice versa Fluids with lower dynamic viscosity would have lower 
resistance from the walls and can flow freely without any disruption.

\subsection{Dispersed phase}

In a droplet-based microfluidic network, a second fluid called dispersed phase and with a dynamic viscosity $\mu_{d}$, is injected into the continuous phase by an independent pump, which in our case is a peristaltic pump. The interfacial tension coefficient between the dispersed phase and the continuous phase. denoted by $\sigma$ [N/m], determines the value of the capillary number $C_{a}$. a dimensionless parameter that describes the relative magnitude of the viscous shear stress compared with the interfacial tension, given by,

$$
C_{a}=\frac{\mu_{c} u_{c}}{\sigma}=\frac{\mu_{c} Q_{c}}{\sigma \pi r_{0}^{2}}
$$

For the dispersed phase to form droplets of regular size the system shall work in the so-called squeezing regime [15][16], which requires

$$
C_{a}<C_{a}^{*} \approx 10^{-2}
$$

This limit, in turn, sets an upper bound $u_{c}^{\prime}$ to the continuous phase speed $u_{c}$, that is,

$$
u_{c}<u_{c}^{*}=\frac{\sigma}{\mu_{c}} 10^{-2}
$$

In this paper, we would assume that the above equation is satisfied and that the dispersed phase takes the form of regular droplets, whose length $l_{d}$ can be approximated as,

$$
l_{d}=r_{0}\left(1+\varepsilon \frac{Q_{d}}{Q_{c}}\right)
$$

Where $\varepsilon$ is the dimensionless parameter of the order of 1 that depends on the absolute value of $Q_{c}$. From mass conservation principle, it follows that the inter-droplet distance is given by,

$$
\delta=l_{d} \frac{Q_{c}}{Q_{d}}=r_{o}\left(\frac{Q_{c}}{Q_{d}}+\varepsilon\right)
$$

The droplet length per inter-distance can then be tune by adjusting the volumetric flow rates $Q_{c}$ and $Q_{d}$ of the continuous and the dispersed phases respectively, if equation 9 holds true. When a droplet is injected into the cross-section, the friction generated with the carrier fluid and the forces produced by the inhomogeneity between the dynamic viscosity of the continuous and the dispersed phases determine a change of the hydraulic resistance of the channel $[17][18][19]$. Mathematically, the variation of resistance produced by a droplet of the length $l_{d}$ in a channel of length $\mathrm{L}$ can be given as follows,

$$
\begin{gathered}
\rho\left(L_{d}\right)=R\left(\mu_{c}, L-\mu_{c}, l_{d}\right)+R\left(\mu_{d}, l_{d}\right) \\
-R\left(\mu_{c}, L\right) \\
=\left(\mu_{d}-\mu_{c}\right) \frac{l_{d} 8}{\pi r_{0}^{4}}
\end{gathered}
$$

such that the overall microfluidic resistance of the channel when crossed by a droplet of length $l_{d}$ is $R\left(\mu_{c}, L\right)+\rho\left(l_{d}\right)$. Note that these relations hold true only when the droplet is large enough to entirely fill the channel section, with an extremely thin stream of continuous phase between the droplet and the channel surfaces. In this case, the speed of the droplet when crossing a channel is basically the same as that of the continuous phase in that channel, that is,

$$
\begin{gathered}
u_{d}=u_{c}=\frac{Q_{c}}{\pi r_{0}^{2}} \\
=\frac{\Delta P}{\pi r_{0}^{2}\left(\frac{8 \mu_{c} L}{\pi r_{0}^{4}}\right)+\left(\mu_{d}+\mu_{c}\right) \frac{l_{d}^{8} 8}{\pi r_{0}^{4}}} \\
u_{d}=\frac{\Delta \operatorname{Pr}_{0}^{2}}{8\left[\mu_{c} L+l_{d}\left(\mu_{c}+\mu_{d}\right)\right]}
\end{gathered}
$$

where $Q_{c}$ is the volumetric flow rate of the continuous phase in the channel, $\Delta P$ is the pressure gradient between the endpoints of the channel. Therefore, the microfluidic resistance in the different part of the network may change over time, depending upon the path followed by the droplets, and their size. The speed of the droplets, however, depends, on the volumetric flow rate of the continuous phase in the different branches that, in turm. depends on the microfluidic resistance of the channels and on the position of the droplet.

\section{Methodology for Automation of the device}

The instruments used for Enceladus biochemical analysis should perform experiments with extreme precision and sensitivity so that they do not contaminate the sample while providing high throughput results. On the other hand, the micro-life package designed should be compact enough to fit in a penetrator. The micro-life detection package includes various analytical and sample stages and instruments through which the sample would pass, and ultimately shows whether there is any microbial activity in the sample or not. The proposed micro-life subsystem consists of a micropump, which is the drive mechanism of the fluid from the heating cup to the filtration chamber. The heating cup is a closely packed model which has a resemblance to a drink coaster. These use ceramic heating coil and once connected to the circuitry, electricity flows and warms up the heating 
element. This element plays an essential role in the case when the collected sample is completely frozen and needs to be analysed in a fluid state. The micropump used to transfer the fluid can maintain a controlled flow rate with high precision and repeatability.

3.1 Tubing and Micropump Requirements

In addition to high precision and accuracy, the pump should be smaller to reduce the overall mass of the system. The micropump proposed for the subsystem was available off the shelf. The pump flow rate required for this application should be in a microliter $(\mu \mathrm{l})$ with a maximum flow rate of approximately $40-60 \mu \mathrm{l} / \mathrm{min}$. The tubing used should not contaminate the sample to be processed. Therefore, polytetrafluoroethylene (PTFE) described as Teflon tubing can often be used for this application. PTFE-coated tubing is transparent, chemically inert. thermally stable and non-toxic, providing chemical resistance and facilitating selfcleaning of parts through non-wvetting. Due to its high flexibility, PTFE coated tubes were used for this application.

Table 2: Micropump parameters and thei specification are reported in this table.

\begin{tabular}{ll}
\hline Parameters & Specification \\
\hline Flow rate & $\begin{array}{l}0.1 \mu \mathrm{l}-40 \mu \mathrm{l} / \mathrm{min} \pm 15 \% \\
\text { (sample at } 25^{\circ} \mathrm{C} \text { ) }\end{array}$ \\
Tubing Material & $\begin{array}{l}\text { PTFE coated silicone tubes } \\
\text { with an inner diameter of }\end{array}$ \\
& $\begin{array}{l}0.5 \mathrm{~mm} \\
\text { Pump pressure }\end{array}$ \\
Motor Control & Stepper motor controlled \\
Dimensions & 33mm $\times 12 \mathrm{~mm} \times 21.5 \mathrm{~mm}$ \\
Pump head & Replaceable \\
\hline
\end{tabular}

The melting point for PTFE is reached at $327^{\circ} \mathrm{C}$, making it suitable for high-temperature applications. With a low flow rate and PTFE silicone tubing, a miniaturized peristaltic pump was the ideal choice for transferring the fluid into the micro-life package. In addition to a flow rate of approximately 40-60 $\mu \mathrm{l}$ and PTFE-coated silicone tubing, the pump should meet the following requirements:

1. Compact size to reduce the total mass of the subsystem,

2. Low to medium pressure range (30KPa or more)

3. Stepper motor control to increase the accuracy of the transferred fluid and

4. Low power requirements and a replaceable pump head.

The micropump from RP-TX series manufactured by Takasago Electric INC. meets the above-mentioned

IAC-19,A1,6,9,x50396 requirements and the specifications are shown in Table 2 It was controlled externally with the help of an ATmega328P Arduino Uno microcontroller.

One of the chambers in the micro-life package contained AlamarBlue reagent. It is ready to use resazurin-based solution that acts as an indicator of cell health by using the reducing power of living cells to quantify viability. The AlamarBlue reagent is non-toxic. a cell-permeable compound of blue colour and virtually non-fluorescent. Upon entry into the living cells, resazurin reduces to resonfin, a red compound that fluoresces strongly. Due to its wide applicability, it can be used with various cell lines, bacteria and fungi Outstanding features include:

1. Robust and reliable performance that results in a large, highly reproducible dynamic range.

2. Highly sensitive reagent with a linear response as it detects as few as 50 cells per well

3. Convenient add-and-read format, that is, no mixing, no washing, no cell lysis.

4. Compatible with either fluorescence- or absorbance-based instrumentation.

5. Measures viability from many diverse cell types including mammalian cells, bacteria. plant, and fungi.

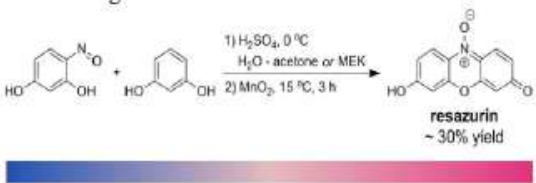

Fig. 2: Chemical formula for alamarBlue dye.

The tubes used must not leach contaminants into the system. In this case, the low bond Eppendorf tube was the preferred choice because it prevents samples from sticking to the tube side. The AlamarBlue reagent and the sample must be thoroughly mixed in a ratio of $10: 90 \mu \mathrm{l}$. The mixing of the components must be done at $4000 \mathrm{rpm}$ for about 60 to 90 seconds as this helps break up the cells. Cell health was monitored by assaying for either enzyme activity, DNA synthesis or the presence of Adenosine triphosphate (ATP). The alamarBlue reagent recognizes the metabolically active cells and aids in the quantitative analysis of cell viability.

To test for viability, the dye was added to the cells in complete medium and incubated for one to four hours. The reading was taken using a fluorescence-based plate reader. For the mixing of the two components, a mechanism was designed that can reciprocate at a high speed of approximately 4000 revolutions per minute (rpm). The mechanism should have support for holding the lysis matrix tubes containing the highly controlled 
size and amount of silica beads used primarily for sample lysis. The selection of the appropriate size of beads to be used for lysis depends on the cell to be inspected.

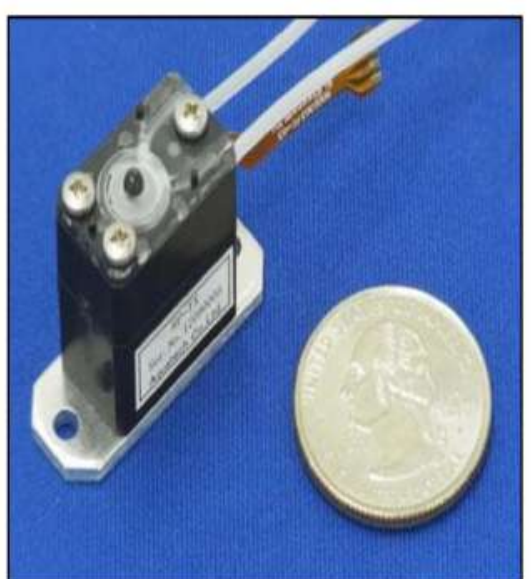

Fig. 3: Image of an RPTX series micropump used in the micro-life package ${ }^{20}$.

The model for mixing of fluid was inspired by the slider-crank mechanism, often known as the quick return mechanism. It converts rotary motion to linear motion and it allows a slower forward stroke of the slider to a faster return stroke. The shaft attached to the crank can be driven by a motor that would provide adequate torque for the slider to reciprocate back and forth.

3.2 Steps for Automation of Microfluidic Network

In order to automate the process, components such as solenoid valves, micropumps and membrane filters were used. Non-contact on/off solenoid valves were chosen as it is suitable for applications sensitive to contamination. These valves stop the flow without contacting the process media by pressing down on the outside of the tubing and operate on electrical signals to automatically start and stop the flow. The valves chosen were normally closed unless actuated. The actuator was directly mounted to the valve body to minimize movement and reduce wear

Before the sample was transferred to the mixing chamber, the sample was filtered through a $5 \mu \mathrm{m}$ filter to remove contaminants. The sample, alamarBlue reagent and the gel beads used for lysing were mixed thoroughly and transferred to the Microbial Activity Micro Assays ( $\mu$-MAMA) to monitor the viability of cells. DNA/RNA sequencing of the cells in the assayed sample can be performed in a sequential manner using readily available samples, as briefly described in figure III. The reagent used in the process are generally stored at $-25^{\circ} \mathrm{C}$ and have a shelf life of about 3 months. From the experiments, it was observed that the reagents work perfectly fine after one year as well. To transfer the environmental sample collected to the next stage, an RPTX micropump was used to drive the sample from the heating cup to one of the microtubes in a fluid state. These microtubes are made of polypropylene with a capacity ranging from $2 \mathrm{ml}$ to $10 \mathrm{ml}$. In addition, they are extremely leaking tight and prevent accidental opening during a transfer of fluids, autoclavable at $121^{\circ} \mathrm{C}$ for 20 minutes and ideal for heating applications.

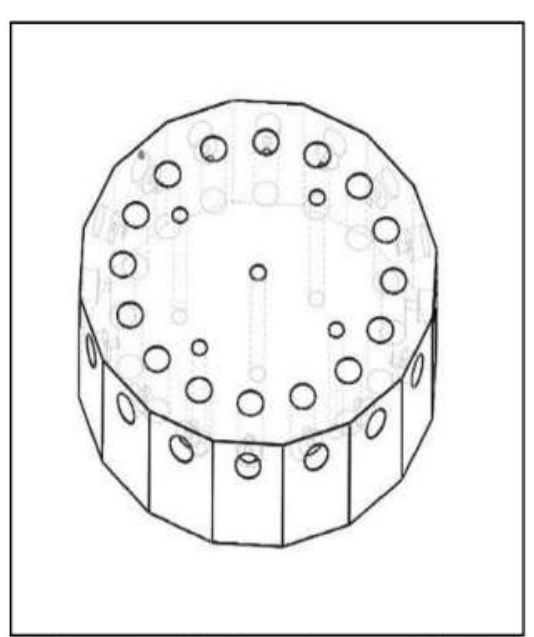

Fig. $4: 3 \mathrm{D}$ model of the microassay with 16 wells separated at an equal distance from each other.

The environmental sample was passed through another filtration step in which the pore size of the filter was 5 microns to remove any contaminants from the environmental sample and then transferred to the microassay through the splitter manifold. The other microtube comprised of the dye reagent was transferred to the microassay with the help of the micropump. Once the dye gets mixed with the environmental sample in appropriate proportion, and if there are any living cells present in the sample, the dye would change its colour and measure the viability of the cells.

The microassay consists of 16 wells with each well designed in such a way that it can hold a sample in a volume of $100 \mu \mathrm{l}$ for testing. These sample stored in the microassay was in the proportion of $90: 10 \mu \mathrm{l}$ with 10 being the sample size of the dye reagent. The total sample size was $1600 \mu \mathrm{l} / \mathrm{well}$ 


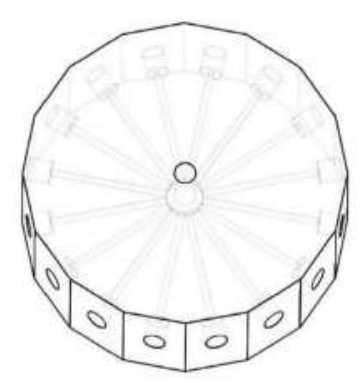

Fig. 5: 3D model of the splitter manifold designed with 10-32 UNF fittings. It consists of one IN port in the centre and 16 OUT ports on the sides.

The microassay was $3 \mathrm{D}$ printed using a biocompatible, autoclavable, transparent rigid resin. The 3D model of the microassay is shown in figure III. Multiple copies of the micro assays were made to test for different samples collected from cryo-environmen analogue sites at the Canadian High Arctic including permafrost, sub-zero saline springs and desert soils. The sample collected from these places is considered to have similar biological or environmental conditions of a celestial body such as Mars or Europa.

To ensure that the same amount of fluid was transferred to the wells of the microassay, a splitter manifold was introduced in between the microassay and the microtubes. This was achieved in such a way that the fluid had to pass from the microtube through a filtration stage to this manifold and from the manifold to the wells of microassay in a controlled manner. The splitter manifold comprises one IN port in the centre and 16 OUT ports on the side. The 3D printed model of the manifold had an equal length to the OUT port from the IN port as shown in figure IV. The same length of tubing was used from the OUT port to get the fluid to the wells of the microassay at the same time. All the OUT ports were designed with 10-32 UNF fittings. Using a splitter manifold with 16 equal lengths OUT ports reduced the complexity of the system and the number of pumps required to transfer the fluid from one stage to the other.

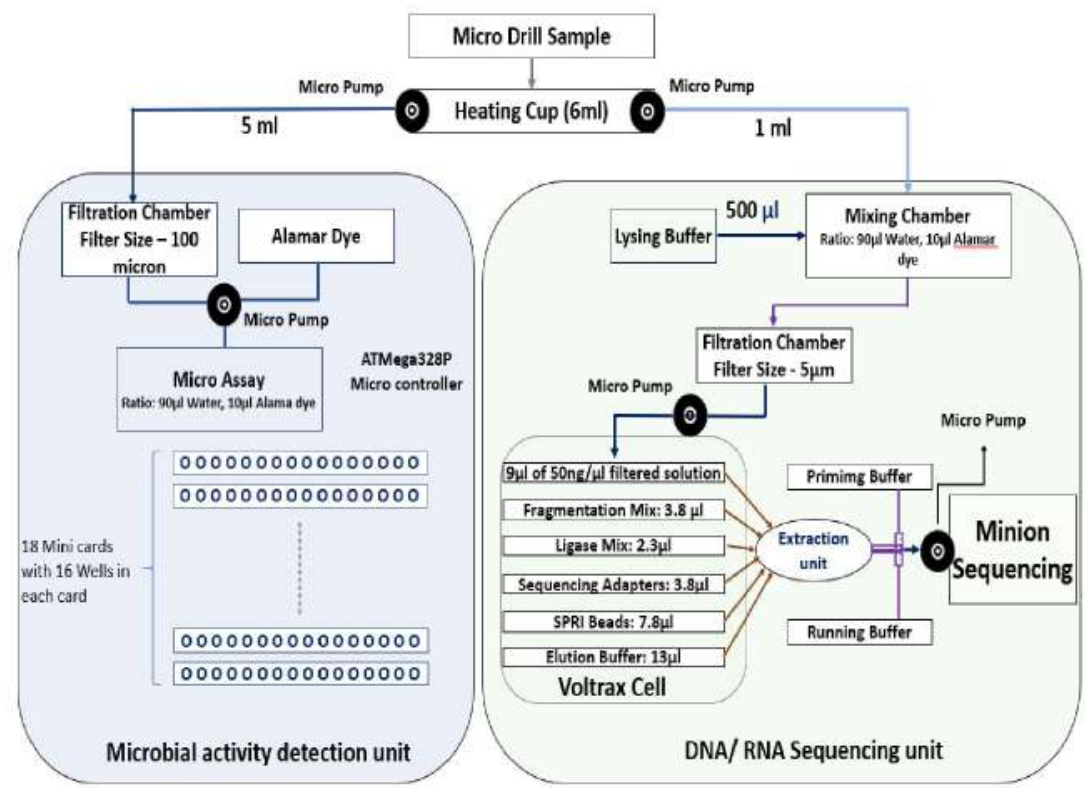

Fig. 6: Flowchart for microfluidic subsystem depicting the steps followed to automate the process. A detailed description of the sample size for the analytical and sample processing functions is also discussed. 


\section{Experimental validation}

To prove the reliability of the model, we carried out a series of experiments and after every test trial, important points were documented that may perhaps lead to the failure of the subsystem. Figure VI shows the algorithm used to automate the process. The experiment was performed in the following sequence. First, the environmental sample was collected by an automated drill and then the sample was passed through the heating cup, which comprises of filtration stage, to ensure the sample that enters the microfluidic system remains in a fluid state for biochemical analysis. When the fluid was acquired in one of the microtubes, Solenoid Valvel (SV1) and MicroPump2 (MP2) were turned on. Now, the fluid was moved from the microtubes to the microassay in through the splitter manifold. Once the fluid reached the wells of the assay, the Solenoid Valve (SV1) \& Micropump2 (MP2) were turned off as mentioned in the flowchart. After SV1 and MP2 get turned off, the MP2 along with SV2 was turned on so that the dye reagent stored in the other microtube was transferred through the circuitry to the $\mu$-MAMA. To ensure that the sample doesn't get contaminated, the $\mu$-MAMA was sealed using a sealan made of silicone rubber and a blank run was acquired.

These silicone rubbers are high-performance platinum cure liquid silicone compound and were suitable for this application as they have a service temperature range of $-53^{\circ} \mathrm{C}$ to $232^{\circ} \mathrm{C}$. After mixing the parts in the required amounts vacuum degaussing was done to eliminate entrapped air. The warmer temperature usually reduces the cure time, however. to obtain better results it was allowed to cure for 16 hours.

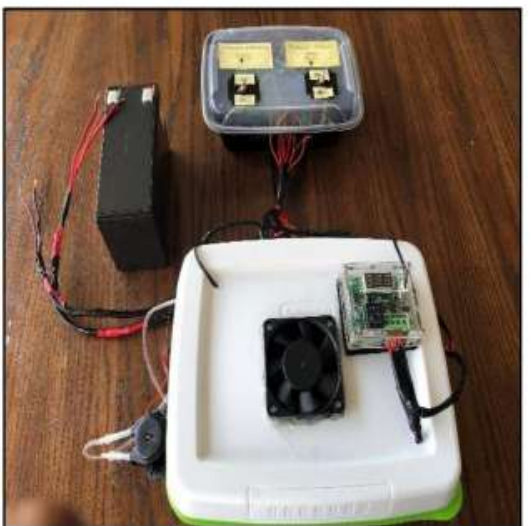

Fig. 8: Working model of the $\mu$-MAMA with the temperature-controlled chamber, control unit and a $12 \mathrm{~V} \mathrm{DC}$ power supply.

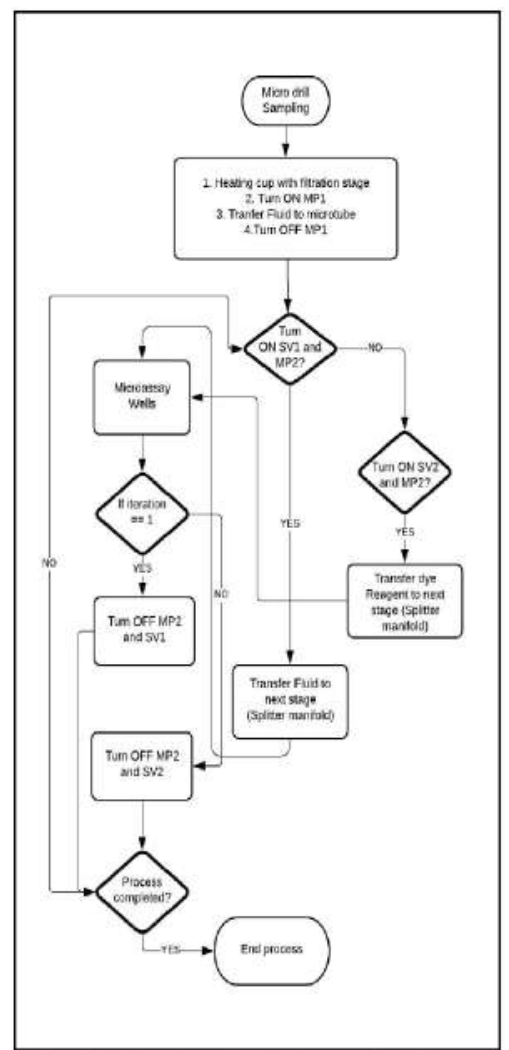

Fig. 7: A flowchart for simulation algorithm.

The subunit was placed in a temperature-controlled chamber which was operated using a temperature control module, a ceramic PTC heater. NTC temperature sensor and a heat sink. With the chosen temperature control module, it was easier to control and monitor the temperature of the chamber and the temperature reading obtained from the module were verified using a non-contact temperature sensor. It was also noted that the module works efficiently for temperature control ranging from $-50^{\circ} \mathrm{C}$ to $110^{\circ} \mathrm{C}$

The working model and the front view of the $\mu$ MAMA prototype are shown in figure VII and VIII. In a similar fashion, the model could be implemented for the second phase of the subunit. To give a brief explanation of this phase, the sample must pass through the filtration step to avoid false positives After the filtration stage, the sample would be pumped to one of the ports of the DNA / RNA Library 
Preparation Manager, i.e. VOLTRAX from Oxford Nanopore.

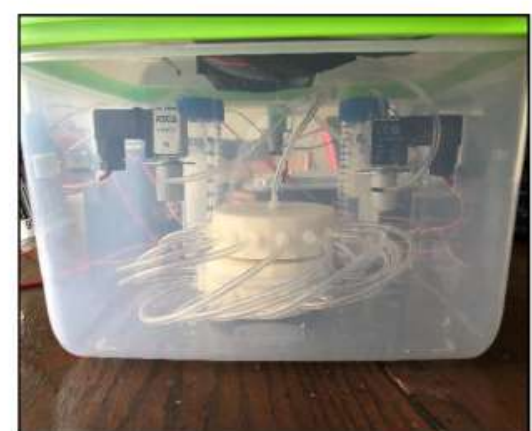

Fig. 9: Closer view of the $\mu$-MAMA prototype.

The VOLTRAX can be controlled via computer control. Once the library is prepared, the sample should be transferred from the extraction port to the minion for sequencing the DNA/RNA for analysis. VOLTRAX was chosen for this application because of its small size and ability to perform library preparation automatically. Once the sample library has been prepared, DNA/RNA can be sequenced and analysed using MinlON. The ultra-long fragment length analysis of DNA/RNA is possible as we can choose the fragment length and can be remotely operated at extreme environments.

Due to the significant increase in the capability represented by the novel microdevice demonstrated here and the automated analysis of the environmental sample increases the possibility of exploring the highly challenging environments. We are currently investigating how this model can be further

\section{References}

II Church. G. Deamer. D.W.. Branton. D.. Baldarelli, R., and Kasianowicz, J., 1998, Characterization of individual polymer molecules based on monomerinterface interactions. U.S. Patent 5795782.

[2] Kasianowicz, J.J., Brandin, E., Branton, D., and Deamer, D. W., 1996, Characterization of individual polynucleotide molecules using a membrane channel. Proceedings of the National Academy of Science of the United States of America, 93(24), pp. 13770-13773.

[3] Majid, S., Yusko, C., Billeh, Y., N., Macrae. M. X., Yang. J., and Mayer, M., 2010, Applications of biological pores in nanomedicine, sensing, and miniaturised so that it can fit in a penetrator. The penetrator model of the microfluidic system would be smaller than the current version. Though the mass of the current subsystem is under the limit but developing an even smaller version would increase the chances to perform tests on other areas of exploration such as cryo-environment analogue sites in the Canadian high Arctic.

\section{Conclusion and Future Work}

In this paper, we described a novel fully functional and integrated microfluidic system prototype that successfully executes automated end-to-end analvsis of the microbial activity in the unknown environmental sample. Besides, we proposed a simple mathematical model to understand the flow physics and mixing of fluid at the macroscopic level. The entire process was controlled remotely without human intervention, that is, from the collection of samples to its biochemical analysis. Based on the experimental results obtained. certain modifications were made to ensure that the system doesn't fail under low-temperature conditions.

In addition, this microfluidic subsystem was designed for deployment on a Mars Rover for working in extreme environments. The efforts to automate the process is crucial as it could serve as a platform for future in-situ investigations to targets such as Europa, Mars and Enceladus where end-to-end automation is required. In future, we would reduce the overall mass and size of the microfluidic system and test it for different habitable cryo-environment analogue sites We would also develop an instrument suite offering the ultimate in speed performance for the lysis of the biological samples to produce highly purified DNA/RNA samples ideal for sequencing.

[4] Venkatesan, B.M. and Bashir, R., 2011. Nanopore sensors for nucleic acid analysis. Nature nanotechnology, 6(10), p.615.

nanoelectronics. Current Opinion in Biotechnology, 21(4), pp. 439-476.

[5] Marshall, M.M., Yang, J. and Hall, A.R., 2012. Direct and transmission milling of suspended silicon nitride membranes with a focused helium ion beam. Scamning, 34(2), pp.101-106.

[6] Venkatesan, B.M., Shah, A.B., Zuo, J.M. and Bashir, R., 2010. DNA sensing using nanocrystalline surface enhanced $\mathrm{Al} 2 \mathrm{O} 3$ nanopore 
sensors. Advanced functional materials, 20(8) pp. $1266-1275$.

[7] Postma, H.W.C., 2010. Rapid sequencing of individual DNA molecules in graphene nanogaps. Nano letters, 10(2), pp.420-425.

[8] Saha, K.K., Drndic, M. and Nikolic, B.K., 2011 DNA base-specific modulation of microampere transverse edge currents through a metallic graphene nanoribbon with a nanopore. Nano letters, $12(1)$, pp.50-55.

[9] Fuerstman, M.J., Garstecki, P. and Whitesides, G.M., 2007. Coding/decoding and reversibility of droplet trains in microfluidic networks. Science, 315(5813), pp.828-832.

[10] Link, D.R., Grasland-Mongrain, E., Duri, A. Sarrazin, F., Cheng. Z., Cristobal, G., Marquez, M and Weitz, D.A., 2006. Electric control of droplets in microfluidic devices. Angewandte Chemie International Edition, 45(16), pp.2556-2560.

[11] Link, D.R., Grasland-Mongrain, E., Duri, A. Sarrazin, F. Cheng, Z. Cristobal. G. Marquez, M and Weitz, D.A., 2006. Electric control of droplets in microfluidic devices. Angewandte Chemie International Edition, 45(16), pp.2556-2560.

[12] Sessoms, D.A., Belloul, M., Engl, W., Roche, M., Courbin, L. and Panizza, P., 2009. Droplet motion in microfluidic networks: Hydrodynamic interactions and pressure-drop measurements. Physical Review E, 80(1), p. 016317

[13] De Leo, E., Donvito, L., Galluccio, L., Lombardo, A., Morabito, G. and Zanoli. L.M., 2013 Communications and switching in microfluidic systems: Pure hydrodynamic control for networking labs-on-a-chip. IEEE transactions on communications, 61(11), pp.4663-4677.

[14] Bulu, A., Chapter 7 Two-Dimensional flow of the Real Fluids, Fluid Mechanics, accessed $3^{\text {rd }}$ August 2019,<https://web.itu.edu.tr/_bulu/fluid_mechani cs files/lecture notes 07.pdf

[15] Thorsen, T., Roberts, R.W., Amold, F.H. and Quake, S.R., 2001. Dynamic pattern formation in a vesicle-generating microfluidic device. Physical review letters, 86(18), p.4163.

[16] Garstecki, P., Fuerstman, M.J., Stone, H.A. and Whitesides, G.M., 2006. Formation of droplets and bubbles in a microfluidic T-junction-scaling and mechanism of break-up. Lab on a Chip, 6(3). pp. $437-446$.

[17] Carlson, A., Do-Quang, M. and Amberg, G., 2010. Droplet dvnamics in a bifurcating channel. International Journal of Multiphase Flow, 36(5), pp.397-405,2

[18] Link, D., R., Anna, S.L., Weitz, D.A. and Stone, H.A., 2004. Geometrically mediated breakup of drops in microfluidic devices. Physical review letters, 92(5), p.054503.

[19] Leshansky, A., M., and Pismen, L., M., 2009. Breakup of drops in a microfluidic $T$ junction. Physics of Fluids, 2l(2), p.023303.

[20] Takasago fluidic systems (TFS), Transfer pump RP-TX Series, Peristaltic pumps, accessed $3^{\text {rd }}$ August 2019,<https://www.takasagofluidics.com/p/pump /s/transfer/RP-TX

[21] Mora, M.F., Greer, F., Stockton, A.M., Bryant, S and Willis, P.A., 2011. Toward total automation of microfluidics for extraterrestial in situ analysis. Analytical chemistry, 83(22), pp.86368641 .

[22] Haque, F., Li, J., Wu, H.C., Liang, X.J. and Guo P., 2013, Solid-state and biological nanopore for real-time sensing of single chemical and sequencing of DNA. Nano today, 8(1), pp.56-74. 